\title{
Measures for Managing Operational Resilience
}

Julia $\mathrm{H}$. Allen

Pamela D. Curtis

July 2011

TECHNICAL REPORT

CMU/SEI-2011-TR-019

ESC-TR-2011-019

CERT $^{\circledR}$ Program

http://www.sei.cmu.edu

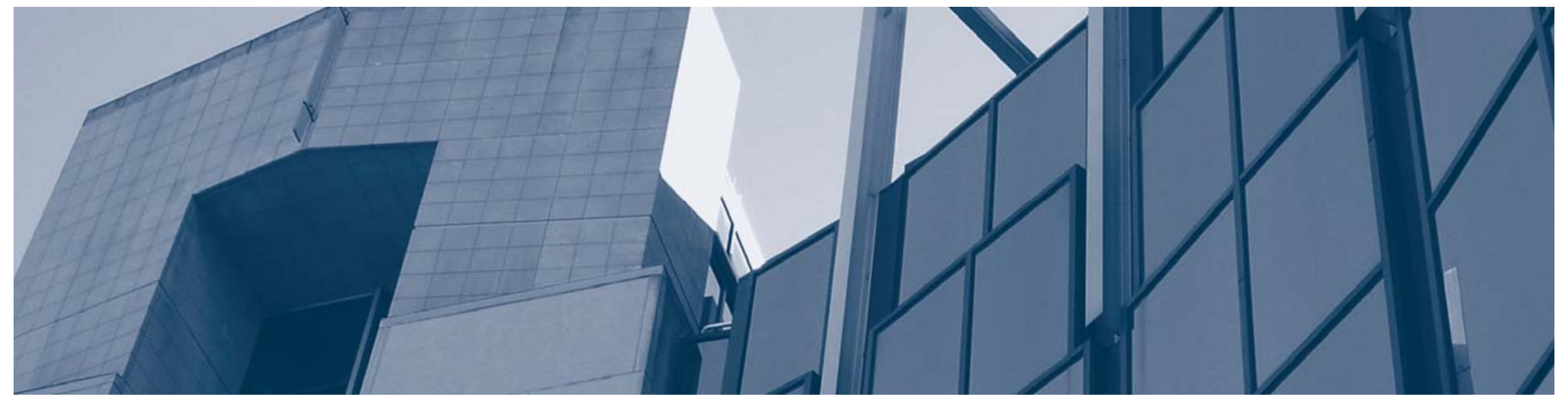


Copyright 2011 Carnegie Mellon University.

This material is based upon work supported by United States Department of Defense under Contract No. FA8721-05-C0003 with Carnegie Mellon University for the operation of the Software Engineering Institute, a federally funded research and development center.

Any opinions, findings and conclusions or recommendations expressed in this material are those of the author(s) and do not necessarily reflect the views of the United States Department of Defense.

This report was prepared for the

SEI Administrative Agent

$\mathrm{ESC} / \mathrm{XPK}$

5 Eglin Street

Hanscom AFB, MA 01731-2100

NO WARRANTY

THIS CARNEGIE MELLON UNIVERSITY AND SOFTWARE ENGINEERING INSTITUTE MATERIAL IS FURNISHED ON AN “AS-IS” BASIS. CARNEGIE MELLON UNIVERSITY MAKES NO WARRANTIES OF ANY KIND, EITHER EXPRESSED OR IMPLIED, AS TO ANY MATTER INCLUDING, BUT NOT LIMITED TO, WARRANTY OF FITNESS FOR PURPOSE OR MERCHANTABILITY, EXCLUSIVITY, OR RESULTS OBTAINED FROM USE OF THE MATERIAL. CARNEGIE MELLON UNIVERSITY DOES NOT MAKE ANY WARRANTY OF ANY KIND WITH RESPECT TO FREEDOM FROM PATENT, TRADEMARK, OR COPYRIGHT INFRINGEMENT.

This material has been approved for public release and unlimited distribution except as restricted below.

Internal use:* Permission to reproduce this material and to prepare derivative works from this material for internal use is granted, provided the copyright and "No Warranty" statements are included with all reproductions and derivative works.

External use:* This material may be reproduced in its entirety, without modification, and freely distributed in written or electronic form without requesting formal permission. Permission is required for any other external and/or commercial use. Requests for permission should be directed to the Software Engineering Institute at permission@sei.cmu.edu.

$\mathrm{CERT}^{\circledR}$ is registered in the U.S. Patent and Trademark Office by Carnegie Mellon University.

For information about SEI publications, please visit the library on the SEI website (www.sei.cmu.edu/library).

* These restrictions do not apply to U.S. government entities. 


\section{Table of Contents}

$\begin{array}{ll}\text { Acknowledgments } & \text { ii }\end{array}$

$\begin{array}{ll}\text { Abstract } & \text { V }\end{array}$

1 Introduction $\quad 1$

$\begin{array}{lll}1.1 & \text { Process Area Measures } & 2\end{array}$

1.2 Information Needs That Drive Resilience Measurement 2

$\begin{array}{lll}1.3 & \text { Report Overview } & 3\end{array}$

2 Top Ten Strategic Measures $\quad 4$

2.1 Organizational Objectives $\quad 4$

2.2 High-Value Services and Assets 4

2.3 Controls $\quad 5$

$\begin{array}{lll}2.4 & \text { Risks } & 5\end{array}$

2.5 Disruptive Events $\quad 6$

$\begin{array}{lll}3 & \text { Introduction to the Resilience Measures } & 7\end{array}$

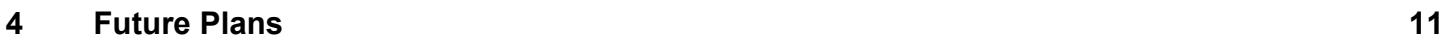

$\begin{array}{llr}\text { Appendix } & \text { Resilience Measures } & 12\end{array}$

$\begin{array}{ll}\text { References } & 71\end{array}$ 
CMU/SEI-2011-TR-019| ii 


\section{Acknowledgments}

The authors would like to thank the reviewers of this report for their thoughtful and valuable comments. Reviewers are members of the CERT Resilient Enterprise Management Team.

- $\quad$ Rich Caralli

- Jim Cebula

- John Haller

- Sam Merrell

- Kevin Partridge

- Barbara Tyson

- David White

- $\quad$ Lisa Young

The authors would also like to thank Noopur Davis of Davis Systems for her review comments. Noopur regularly contributes to REM team measurement work and is the co-author of Measuring Operational Resilience Using the CERT Resilience Management Model [Allen 2010]. 
CMU/SEI-2011-TR-019| iv 


\section{Abstract}

How resilient is my organization? Have our processes made us more resilient? Members of the CERT ${ }^{\circledR}$ Resilient Enterprise Management (REM) team are conducting research to address these and other related questions. The team's first report, Measuring Operational Resilience Using the CERT Resilience Management Model, defined high-level objectives for managing an operational resilience management (ORM) system, demonstrated how to derive meaningful measures from those objectives, and presented a template for defining resilience measures, along with example measures.

In this report, REM team members suggest a set of top ten strategic measures for managing operational resilience. These measures derive from high-level objectives of the ORM system defined in the CERT ${ }^{\circledR}$ Resilience Management Model, Version 1.1 (CERT®-RMM). The report also provides measures for each of the 26 process areas of CERT-RMM, as well as a set of global measures that apply to all process areas. This report thus serves as an addendum to CERT-RMM Version 1.1 .

Since CERT-RMM practices map to bodies of knowledge and codes of practice such as ITIL, COBIT, ISO2700x, BS25999, and PCI DSS, the measures may be useful for measuring security, business continuity, and IT operations management processes, either as part of adoption of CERTRMM or independent of it. 
CMU/SEI-2011-TR-019| vi 


\section{Introduction}

The purpose of this technical report is to present operational resilience measures developed through ongoing research that was first reported in Measuring Operational Resilience Using the CERT Resilience Management Model [Allen 2010].

$\mathrm{CERT}^{\circledR}-\mathrm{RMM}^{1}$ version 1.1 defines operational resilience as "the organization's ability to adapt to risk that affects its core operational capacities. Operational resilience is an emergent property of effective operational risk management" [Caralli 2011].

Operational risk management is supported and enabled by the disciplines of security, business continuity, and some aspects of IT operations. CERT-RMM provides a process view of resilience by describing the practices of these disciplines as part of larger enterprise processes. A process can be defined, communicated, and controlled. The desired goals and outcomes of the process can be identified, success in reaching those goals and outcomes can be measured, and gaps can be identified and addressed.

Operational resilience supports the ability of services and their associated assets (information, technology such as systems and software, facilities, and people) to achieve their mission. An operationally resilient service is a service that can meet its mission under times of disruption or stress and can return to normalcy when the disruption ${ }^{2}$ or stress is eliminated. A service is not resilient if it cannot return to normalcy after being disrupted, even if it can temporarily withstand adverse circumstances.

Resilience objectives for services and assets are achieved through an operational resilience management (ORM) system. An ORM system includes all of the processes necessary to manage operational resilience, along with their associated and supporting plans, programs, procedures, practices, and people. In our first report, we defined high-level objectives for managing an ORM system and demonstrated how to derive meaningful measures from those objectives. For example, one high-level objective identified was, "Demonstrate that the ORM system sustains high-value services and associated assets during and following a disruptive event." One measure defined for that objective was, "For disrupted high-value services with a service continuity plan, percentage of services that delivered service as intended throughout the disruptive event" [Allen 2010].

Linking to high-level objectives can help establish measurement priorities at the enterprise level. Some strategic measures provide meaningful information for business decision making, and many can be used to guide the day-to-day operational resilience of services and their associated assets. In Chapter 2 of this report, we take another look at deriving measures from high-level objectives to suggest a list of the top ten strategic measures for managing operational resilience.

$1 \quad \mathrm{CERT}^{\circledR}$ is registered in the U.S. Patent and Trademark Office by Carnegie Mellon University.

2 "Disruption" in this definition applies to a disturbance that does not exceed the service's operational limit. A catastrophic loss of infrastructure would not be considered a disruption. 


\subsection{Process Area Measures}

The bulk of this report, however, focuses on measurement priorities for each of the 26 CERTRMM process areas (PAs). ${ }^{3}$ These measures were derived from the specific practices and subpractices of the PAs and are intended to measure either the extent of the practices' implementation or their effectiveness in improving operational resilience.

Some of the measures appear in simple list form in Generic Goal 2, Generic Practice 8 (GG2.GP8), "Monitor and Control the Process," in their respective PAs in Version 1.1 of the model, and they are updated and expanded in this report. Other measures are new as of this report. They are process measure examples for GG2.GP8 subpractice 2, "Review accomplishments and results of the process against the plan for performing the process." es in the model are those that apply to every PA; thus every PA has a GG2.GP8, but the measure examples are specific to each PA.

The generic goals and practices in CERT-RMM are indicators of progressive levels of capability. Generic goal 1 in any PA relates to achieving performance of the specific practices of that PA (capability level 1). Generic goal 2 assumes that the specific practices are being performed and provides guidance for higher capability practices such as planning the process and measuring performance against the plan. For those who are using the model, the measures can be used to help achieve capability level 2 in any given PA. For those who are not using the model, the measures can be useful for measuring security, business continuity, and IT operations management processes because CERT-RMM practices map to bodies of knowledge and codes of practice such as ITIL, COBIT, ISO2700x, BS25999, and PCI DSS.

We expect many of these tactical measures at the PA level will be combined to inform more strategic measures, which will in turn demonstrate the extent to which operational resilience objectives are (or are not) being met. So there is a need for both types of measures.

\subsection{Information Needs That Drive Resilience Measurement}

The measures in this report result from research by the authors and other CERT Program staff members at the SEI to assist business leaders in addressing key questions they may be asked (or may ask themselves). The measures inform the answers to these questions:

- How resilient is my organization?

- Is it resilient enough?

- How resilient does it need to be?

Some further interpretations of these questions might include the following:

- Do I need to worry about operational resilience?

3 A process area is a cluster of related practices in an area that, when implemented collectively, satisfies a set of goals considered essential for that area. For example, the goals of the CERT-RMM People Management PA are "Establish Vital Staff," "Manage Risks Associated with Staff Availability," and "Manage the Availability of Staff."

4 Although the authors have attempted to be thorough in developing useful measures related to the CERT-RMM PAs, there may be other measures that will be meaningful for your organization. Measuring Operational Resilience Using the CERT Resilience Management Model [Allen 2010] provides guidance and a template for developing resilience measures. 
- If my services are disrupted, will it make the news? Will I end up in court? In jail? Will I be able to stay in business?

- Do I meet compliance requirements?

- How resilient am I compared to my competition?

- Do I need to spend more on resilience? If so, on what?

- What am I getting for what I've already spent?

- What is the business value of being more resilient?

The key questions being addressed by this research project include

- What should I be measuring to determine if I am achieving my performance objectives for operational resilience?

- Have our processes made us more resilient?

- Given that measurement is expensive, how can I identify measures that will most effectively inform decisions and affect behavior?

\subsection{Report Overview}

As mentioned above, Chapter 2 of this technical report introduces a set of "top ten" strategic measures that have been derived from the objectives for operational resilience in Measuring $O p$ erational Resilience Using the CERT Resilience Management Model [Allen 2010]. These measures have received positive feedback at several conferences. We will continue to update and refine these measures as the basis for additional measures that provide a top-down view.

The Appendix of this report updates all process implementation and effectiveness measures listed in Generic Goal 2, Generic Practice 8 (GG2.GP8), "Monitor and Control the Process," in each of the 26 PAs of CERT-RMM v1.1. These process implementation and effectiveness measures provide a bottom-up, tactical view.

The purpose of this update is to improve completeness (with respect to covering the specific goals and practices in CERT-RMM v1.1), consistency, and clarity. In addition, the report adds a new set of global measures that apply to all PAs. These global measures have been deleted from each of the individual PAs. The tables presented in this report serve as updates and an addendum to CERT-RMM v1.1 and replace corresponding elaboration tables in GG2.GP8 subpractice 2 for each PA.

Some of the measures use concepts and terminology from the model. Please refer to the glossary and relevant PA sections in CERT-RMM ([Caralli 2011] or www.cert.org/resilience/rmm.html) for clarification as needed. 


\section{Top Ten Strategic Measures}

Operational resilience strategic measures help ensure that any measurement of operational resilience directly supports the achievement of business objectives. One of the many pitfalls of unsuccessful measurement programs is collecting, analyzing, and reporting data that does not contribute to informing decisions or changing behavior. Often measurement programs collect and report measures of "type count" (such as number of incidents, number of systems with patches installed, number of people trained) with little meaningful context for how these measures will be used. ${ }^{5}$ By having a set of strategic measures, we can map those measures to the most useful measures at the individual PA level and develop criteria to determine which PA-level measures best address the questions posed in the Introduction. In addition, measurement can be expensive, and organizations should be judicious in selecting measures that form the foundation of their measurement program.

The strategic objectives for an ORM system are described in the next five sections. Each is currently supported by two measures. For further details, refer to Section 2.3 of Measuring Operational Resilience Using the CERT Resilience Management Model [Allen 2010].

\subsection{Organizational Objectives}

Objective: The ORM system derives its authority from and directly traces to organizational drivers. Organizational drivers include strategic objectives and critical success factors (refer to the Enterprise Focus process area [Caralli 2011]). An alternative way of stating this might be "The ORM system derives its authority from a directive given by a senior, high-level executive." This could be considered one form of organizational driver.

Measure 1: Percentage of resilience activities that do not directly (or indirectly) support one or more organizational objectives. An activity can be a project, task, performance objective, or investment, and represents some meaningful decomposition of the resilience program.

Measure 2: For each resilience activity, number of organizational objectives that require it to be satisfied (goal is $=$ or $>1$ )

Supporting measures that address the relationship between organizational objectives and resilience are contained in the Appendix table for Enterprise Focus (EF).

\subsection{High-Value Services and Assets}

Objective: The ORM system satisfies resilience requirements that are assigned to high-value services and their associated assets. An alternative way of stating this might be "The ORM system satisfies governance, compliance, policy, framework, assessment, and reporting requirements." These could all be considered expressions of enterprise resilience requirements.

While readers of this report will see measures of this type in the Appendix, they are presented in the context of one or more process areas and are often used as the basis for calculating derived measures. 
Measure 3: Percentage of high-value services that do not_satisfy their assigned resilience requirements. (Operational resilience requirements are a derivation of the traditionally described security objectives of confidentiality, availability, and integrity. They may also include privacy requirements.) A companion measure would be to measure a specific service of interest, ensuring that criteria for selecting such a service are defined.

Measure 4: Percentage of high-value assets (information, technology, facilities, and people) that do not satisfy their assigned resilience requirements. Examples of assets are network infrastructure, a specific application, a database, a data center, and a lead system administrator.

Supporting measures that address resilience requirements for services are contained in the Appendix table for EF. Supporting measures that address resilience requirements for assets are contained in the following Appendix tables by asset type:

- Asset Definition and Management (ADM) - general

- Environmental Control (EC) - facilities

- Knowledge Information and Management (KIM) - information

- $\quad$ People Management (PM) - people

- Technology Management (TM) - technology

\subsection{Controls}

Objective: Via the internal control system, ${ }^{6}$ the ORM system ensures that controls for protecting and sustaining high-value services and their associated assets operate as intended.

Measure 5: Percentage of high-value services with controls that are ineffective or inadequate. This may include unsatisfied control objectives, unmet resilience requirements, missing controls, and outstanding assessment and audit problems above threshold without remediation plans.

Measure 6: Percentage of high-value assets with controls that are ineffective or inadequate

Supporting measures that address controls in general are contained in the Appendix table for Controls Management (CTRL). Supporting measures that address controls for assets are contained in the Appendix tables by asset type as noted in Section 2.2, Measure 4.

\subsection{Risks}

Objective: The ORM system manages operational risks to high-value assets that could adversely affect the operation and delivery of high-value services.

Measure 7: Confidence factor that risks from all sources that need to be identified have been identified. A detailed template for this measure appears in Section 4.1.1 of Measuring Operational Resilience Using the CERT Resilience Management Model [Allen 2010].

Measure 8: Percentage of risks with impact above threshold. This should include risks without mitigation plans, risks that are not effectively mitigated by their mitigation plans, and risks that have not been reviewed in the required time frame.

6 The internal control system includes the methods, policies, and procedures used to protect and sustain assets at a level commensurate with their role in supporting high-value services. 
Supporting measures that address risks in general are contained in the Appendix table for Risk Management (RISK). Supporting measures that address risks for assets are contained in the Appendix tables by asset type as noted in Section 2.2, Measure 4.

\subsection{Disruptive Events}

Objective: In the face of realized risk, the ORM system ensures the continuity of essential operations of high-value services and their associated assets. Realized risk may include an incident, a break in service continuity, or a man-made or natural disaster or crisis.

Measure 9: Probability of delivered service through a disruptive event

Measure 10: For disrupted, high-value services with a service continuity plan, percentage of services that did not deliver service as intended throughout the disruptive event

Consider using "near misses" and "incidents avoided" as predictors of successful disruptions in the future.

Supporting measures that address service continuity are contained in the Appendix table for Service Continuity (SC). Supporting measures that address incident management are contained in the Appendix table for Incident Management and Control (IMC). 


\section{Introduction to the Resilience Measures}

The following process was used to generate the tables of resilience measures presented in the Appendix:

1. Starting with existing GG2.GP8 subpractice 2 process area measures for each PA in CERTRMM v1.1, the type of information that each measure addresses was designated (see the Column 3 description below). Measures were then ordered in a logical progression by type of information.

2. The new Measure Type and Base or Derived columns (Columns 4 and 5, respectively) were populated for each existing measure.

3. The PA specific goals and specific practices were carefully reviewed, and measures were added, corrected, combined, or eliminated, as needed. Each measure was mapped to the specific goal(s) and practice(s) that it informs (Column 6).

4. All measures were edited for clarity and consistency and to eliminate redundancy, separate compound measures, and eliminate measures of insufficient information value.

5. Measures that are global in nature were identified (that is, ones that apply to all PAs). A new table of global measures was created, and the global measures were deleted from the tables of PA-specific measures. Global measures were defined for the generic goals and practices that accompany each process area.

6. All measures tables were reviewed by at least two reviewers.

This process was started for three process areas in Measuring Operational Resilience Using the CERT Resilience Management Model, specifically Knowledge and Information Management (KIM), Incident Management and Control (IMC), and Risk Management (RISK). Refer to Section 4.1 of that document for information on these three process areas in the context of selected CERTRMM ecosystems (a collection of process areas, relationships, goals, and practices that contribute to a specific objective, such as the management of risk).

Each table is organized as follows (refer to the Appendix):

\section{Column 1: ID}

The ID field is a unique, sequential identifier that is assigned to each measure. We organized measures in a logical progression, which often matches the order of the specific goals and practices for the specific PA. The Column 3 entry typically determines the order of the measures.

\section{Column 2: Measure}

This field contains the measure or, in some cases, a set of related measures.

\section{Column 3: Type of Information}

The intent of this field is to identify several standard types of information within each PA that the measure informs. These may be CERT-RMM work products (such as asset inventory and asset controls) or activities (such as change management and obligation satisfaction). Type of informa- 
tion entries support some level of affinity grouping of related measures and may be used in the future to reduce or aggregate related measures.

\section{Column 4: Measure Type}

Measures can be one of three types:

- Implementation measures answer the question, Is this process, activity, or practice being performed? Such measures provide no information regarding the contribution (or lack thereof) that the activity is making to improved operational resilience. The measures presented in this report are predominantly implementation measures. This is as expected for this stage of our research project, given that such measures describe an organization's behavior as it is starting to improve its operational resilience management processes (referred to in CERT-RMM as capability levels 1 and 2).

- Effectiveness measures answer the questions, How good is the work product or outcome of the process, activity, or practice? Does it achieve the intended result? Effectiveness measures are typically of much greater interest than implementation ones. Many of them derive from one or more implementation measures.

- $\quad$ Process performance measures answer the questions, Is the process performing as expected? Is it efficient? Can it be planned? Is it predictive? Is it in control? There are only a few process performance measures described in this report. This is as expected for this stage of our research project, given that organizations focus on institutionalizing defined processes at a later stage of their resilience improvement activities (referred to in CERT-RMM as capability level 3).

On occasion, the Measure Type field states "implementation, possibly effectiveness." Such measures may be potential candidates for effectiveness measures but require additional interpretation and analysis.

For further details on these definitions, refer to Section 3.1.3 of Measuring Operational Resilience Using the CERT Resilience Management Model [Allen 2010].

\section{Column 5: Base or Derived}

This field indicates whether the measure is a base measure or a derived measure, defined as follows:

- A base measure is a directly observable attribute of an asset, service, or resilience process. A measure quantifies an attribute; a person's height can be measured in feet and inches, service response time can be measured in seconds or minutes, and process elapsed time can be measured in days or months. A base measure is thus defined by fundamental units that are not composed of any other units and is functionally independent of other measures. Base measures can be one of four types: count, cost or effort, schedule, or defects. Most of the base measures presented in the tables are of type count (number of) or of type schedule (elapsed time since or total calendar time).

- A derived measure is a mathematical function of two or more base and/or derived measures. Examples of resilience derived measures are percentage of incidents that exploited existing vulnerabilities with known solutions, patches, or workarounds; percentage of information as- 
sets without assigned resilience requirements; and change in number of identified risks that exceed risk parameters.

For further details on these definitions, refer to Section 3.1 of Measuring Operational Resilience Using the CERT Resilience Management Model [Allen 2010].

\section{Column 6: Applicable SG.SP}

This field contains the mapping of the measure to applicable CERT-RMM specific goals and practices. The identified SGs and SPs served as the source for the measure. Occasionally the field entry states "none." This indicates a measure that derives from another source (such as a CERTRMM appraisal) and is not specifically called for in the model.

While the tables are presented in alphabetical order by PA acronym, measures were often developed across related sets of PAs that share common specific goals and practices. Three noteworthy "clusters" of PAs are the asset cluster, the risk cluster, and the controls cluster. Readers of the measures contained in these tables will see commonality and repetition of certain measures. While repetition adds to the effort to maintain measures tables, we want to ensure that each table stands alone to the greatest extent possible (without having to draw upon measures in other PA tables). These clusters include the following process areas:

- Asset cluster: This cluster includes the following related process areas for the identification and management of assets:

- ADM: Asset Definition and Management

- EC: Environmental Control

- KIM: Knowledge and Information Management

- PM: People Management

- TM: Technology Management

A number of measures that appear in ADM are repeated in EC, KIM, PM, and TM, qualified by asset type (facilities, information, people, and technology, respectively).

- $\quad$ Risk cluster: This cluster includes the following related process areas for the identification and management of risks to assets:

- RISK: Risk Management

- EC: Environmental Control

- KIM: Knowledge and Information Management

- PM: People Management

- TM: Technology Management

This cluster also includes the External Dependencies (EXD) PA for the identification and management of risks to external entities and external dependencies. A number of measures that appear in RISK are repeated in EC, KIM, PM, and TM as well as EXD.

- Controls cluster: This cluster includes the following related process areas for the identification and management of controls for assets:

- CTRL: Controls Management 
- EC: Environmental Control

- KIM: Knowledge and Information Management

- PM: People Management

- TM: Technology Management

A number of measures that appear in CTRL are repeated in EC, KIM, PM, and TM, qualified by asset type (facilities, information, people, and technology, respectively).

All measures are intended to be repeatedly collected and reported over time. Often changes in measures from one reporting period to the next and trends over time are of greatest interest. Thus, readers will not see specific references to time durations or periods of time in these measures other than the occasional use of "elapsed time." 


\section{Future Plans}

This research project will continue through FY12 (October 2011 through September 2012). Future plans include populating a database with all of the measures identified in this report. Through use of this database, measures can be easily maintained and mapped to other CERT-RMM artifacts such as specific goals and practices and CERT-RMM Compass questions. ${ }^{7}$

The research team will perform the following tasks to identify additional measures and updates to existing measures:

- $\quad$ Review CERT-RMM appraisal results, Compass review results, and results of other CERTRMM assessment efforts.

- Develop an approach (and templates) for defining CERT-RMM processes at the implementation level and use these process definitions to define additional measures.

- Perform a review and analysis of all measures of measure type effectiveness to identify gaps.

- Identify which process area measures provide information supporting the top ten strategic measures. Identify criteria for prioritizing process measures based on strategic measures.

- Obtain guidance and feedback from members of the CERT-RMM Users Group.

The team will also develop additional measures templates for key measures (refer to Section 3.3 of Measuring Operational Resilience Using the CERT Resilience Management Model [Allen 2010]).

The authors of this report welcome your comments and feedback. We can be contacted at jha@cert.org and pdc@cert.org.

http://www.cert.org/resilience/rmm_compass.html 


\section{Appendix Resilience Measures}

The table of global measures that applies to all process areas appears first. It is following by 26 tables of process-area-specific measures. Each table of measures is preceded by the name of the process area, its purpose, and a summary of its specific goals and practices. This content is taken directly from the $C E R T^{\mathbb{B}}$ Resilience Management Model: A Maturity Model for Managing Operational Resilience [Caralli 2011].

\section{Global Measures}

Organizations deploy generic goals and practices to attain successively improving degrees of process institutionalization and capability maturity for operational resilience management. These practices exhibit the organization's commitment and ability to perform operational resilience management processes, as well as its ability to measure performance and verify implementation.

\section{Summary of Generic Practices for Generic Goal 2 Institutionalize a Managed Process}

GG2.GP1 Establish Process Governance

GG2.GP2 Plan the Process

GG2.GP3 Provide Resources

GG2.GP4 Assign Responsibility

GG2.GP5 Train People

GG2.GP6 Manage Work Product Configurations

GG2.GP7 Identify and Involve Relevant Stakeholders

GG2.GP8 Monitor and Control the Process

GG2.GP9 Objectively Evaluate Adherence

GG2.GP10 Review Status with Higher-Level Managers

\section{Measures}

\begin{tabular}{|l|l|l|l|l|l|}
\hline ID & Measure & $\begin{array}{l}\text { Type of In- } \\
\text { formation }\end{array}$ & $\begin{array}{l}\text { Measure } \\
\text { Type }\end{array}$ & $\begin{array}{l}\text { Base or } \\
\text { Derived }\end{array}$ & $\begin{array}{l}\text { Applicable } \\
\text { GG.GP }\end{array}$ \\
\hline G-M1 & $\begin{array}{l}\text { percentage of higher-level managers who have } \\
\text { documented resilience objectives that are } \\
\text { reviewed as part of the normal performance } \\
\text { review process }\end{array}$ & governance & impl & derived & $\begin{array}{l}\text { GG2.GP1 } \\
\text { GG2.GP8 }\end{array}$ \\
\hline G-M2 & $\begin{array}{l}\text { elapsed time since resilience-related com- } \\
\text { liance obligations were reviewed by higher- } \\
\text { level managers }\end{array}$ & governance & impl & derived & $\begin{array}{l}\text { GG2.GP1 } \\
\text { GG2.GP8 }\end{array}$ \\
\hline G-M3 & $\begin{array}{l}\text { elapsed time since resilience-related controls } \\
\text { in the context of the organization's internal } \\
\text { control system were reviewed by higher-level } \\
\text { managers }\end{array}$ & $\begin{array}{l}\text { governance; } \\
\text { status }\end{array}$ & impl & derived & $\begin{array}{l}\text { GG2.GP1 } \\
\text { GG2.GP8 } \\
\text { GG2.GP10 }\end{array}$ \\
\hline G-M4 & $\begin{array}{l}\text { elapsed time since higher-level managers } \\
\text { reviewed the priorities of services and asso- } \\
\text { ciated assets and provided updated guidance }\end{array}$ & $\begin{array}{l}\text { governance; } \\
\text { status }\end{array}$ & impl & derived & $\begin{array}{l}\text { GG2.GP1 } \\
\text { GG2.GP8 } \\
\text { GG2.GP10 }\end{array}$ \\
\hline
\end{tabular}




\begin{tabular}{|c|c|c|c|c|c|}
\hline ID & Measure & $\begin{array}{l}\text { Type of In- } \\
\text { formation }\end{array}$ & $\begin{array}{l}\text { Measure } \\
\text { Type }\end{array}$ & $\begin{array}{l}\text { Base or } \\
\text { Derived }\end{array}$ & $\begin{array}{l}\text { Applicable } \\
\text { GG.GP }\end{array}$ \\
\hline G-M5 & $\begin{array}{l}\text { elapsed time since audit reports on resilience- } \\
\text { related controls in the context of the organiza- } \\
\text { tion's internal control system were reviewed by } \\
\text { appropriate committees }\end{array}$ & $\begin{array}{l}\text { governance; } \\
\text { status }\end{array}$ & impl & derived & $\begin{array}{l}\text { GG2.GP1 } \\
\text { GG2.GP8 } \\
\text { GG2.GP10 }\end{array}$ \\
\hline G-M6 & $\begin{array}{l}\text { elapsed time since higher-level managers } \\
\text { reviewed the performance and effectiveness of } \\
\text { the operational resilience management system } \\
\text { and its processes and provided any necessary } \\
\text { course correction }\end{array}$ & $\begin{array}{l}\text { governance; } \\
\text { status }\end{array}$ & impl & derived & $\begin{array}{l}\text { GG2.GP1 } \\
\text { GG2.GP8 } \\
\text { GG2.GP10 }\end{array}$ \\
\hline G-M7 ${ }^{8}$ & $\begin{array}{l}\text { percentage of policies }{ }^{9} \text { that are met (no viola- } \\
\text { tions, all exceptions approved) }\end{array}$ & policy & impl & derived & $\begin{array}{l}\text { GG2.GP1 } \\
\text { GG2.GP8 }\end{array}$ \\
\hline G-M8 & $\begin{array}{l}\text { percentage of policies (and/or procedures) that } \\
\text { require updates to reflect CERT-RMM process } \\
\text { area goals and practices }\end{array}$ & policy & impl & derived & $\begin{array}{l}\text { GG2.GP1 } \\
\text { GG2.GP8 }\end{array}$ \\
\hline G-M9 & $\begin{array}{l}\text { percentage of CERT-RMM practices (based on } \\
\text { a specific model scope } \mathrm{e}^{10} \text { ) that are required as } \\
\text { a result of policies (and/or procedures) }\end{array}$ & policy & impl & derived & $\begin{array}{l}\text { GG2.GP1 } \\
\text { GG2.GP8 }\end{array}$ \\
\hline G-M10 & $\begin{array}{l}\text { number of policy violations, aggregate and by } \\
\text { policy }\end{array}$ & policy & impl & $\begin{array}{l}\text { base of } \\
\text { type count }\end{array}$ & $\begin{array}{l}\text { GG2.GP1 } \\
\text { GG2.GP8 }\end{array}$ \\
\hline G-M11 & $\begin{array}{l}\text { percentage of policy exceptions approved, } \\
\text { aggregate and by policy }\end{array}$ & policy & impl & derived & $\begin{array}{l}\text { GG2.GP1 } \\
\text { GG2.GP8 }\end{array}$ \\
\hline G-M12 & $\begin{array}{l}\text { percentage of process activities that are on } \\
\text { track per plan }\end{array}$ & $\begin{array}{l}\text { process plan; } \\
\text { process activ- } \\
\text { ities }\end{array}$ & impl & derived & $\begin{array}{l}\text { GG2.GP2 } \\
\text { GG2.GP8 }\end{array}$ \\
\hline G-M13 & $\begin{array}{l}\text { difference in planned versus actual schedule to } \\
\text { perform the process }\end{array}$ & $\begin{array}{l}\text { process plan; } \\
\text { process activ- } \\
\text { ities }\end{array}$ & $\begin{array}{l}\text { impl; possi- } \\
\text { bly process } \\
\text { performance }\end{array}$ & derived & $\begin{array}{l}\text { GG2.GP2 } \\
\text { GG2.GP8 }\end{array}$ \\
\hline G-M14 & $\begin{array}{l}\text { percentage of process activities approved but } \\
\text { not implemented (due to, for example, sche- } \\
\text { dule and resource constraints) }\end{array}$ & $\begin{array}{l}\text { process plan; } \\
\text { process activ- } \\
\text { ities }\end{array}$ & impl & derived & $\begin{array}{l}\text { GG2.GP2 } \\
\text { GG2.GP8 }\end{array}$ \\
\hline G-M15 & number of scope changes to process activities & $\begin{array}{l}\text { process plan; } \\
\text { process activ- } \\
\text { ities }\end{array}$ & impl & $\begin{array}{l}\text { base of } \\
\text { type count }\end{array}$ & $\begin{array}{l}\text { GG2.GP2 } \\
\text { GG2.GP8 }\end{array}$ \\
\hline G-M16 & $\begin{array}{l}\text { change in resource needs to support the } \\
\text { process }\end{array}$ & $\begin{array}{l}\text { process plan; } \\
\text { resources }\end{array}$ & $\begin{array}{l}\text { impl; possi- } \\
\text { bly effective- } \\
\text { ness }\end{array}$ & derived & $\begin{array}{l}\text { GG2.GP3 } \\
\text { GG2.GP8 }\end{array}$ \\
\hline G-M17 & $\begin{array}{l}\text { percentage of process activities for which } \\
\text { funds have been allocated as planned }\end{array}$ & $\begin{array}{l}\text { process plan; } \\
\text { resources }\end{array}$ & impl & derived & $\begin{array}{l}\text { GG2.GP3 } \\
\text { GG2.GP8 }\end{array}$ \\
\hline G-M18 & $\begin{array}{l}\text { percentage of process activities for which staff } \\
\text { have been allocated as planned }\end{array}$ & $\begin{array}{l}\text { process plan; } \\
\text { resources }\end{array}$ & impl & derived & $\begin{array}{l}\text { GG2.GP3 } \\
\text { GG2.GP8 }\end{array}$ \\
\hline G-M19 & $\begin{array}{l}\text { difference in planned versus actual staff } \\
\text { trained to perform the process }\end{array}$ & $\begin{array}{l}\text { process plan; } \\
\text { resources }\end{array}$ & $\begin{array}{l}\text { impl; possi- } \\
\text { bly process } \\
\text { performance }\end{array}$ & derived & $\begin{array}{l}\text { GG2.GP3 } \\
\text { GG2.GP8 }\end{array}$ \\
\hline G-M20 & cost to support the process & $\begin{array}{l}\text { process plan; } \\
\text { resources }\end{array}$ & $\begin{array}{l}\text { impl; possi- } \\
\text { bly effective- } \\
\text { ness }\end{array}$ & derived & $\begin{array}{l}\text { GG2.GP3 } \\
\text { GG2.GP8 }\end{array}$ \\
\hline G-M21 & $\begin{array}{l}\text { difference in planned versus actual cost to } \\
\text { perform the process }\end{array}$ & $\begin{array}{l}\text { process plan; } \\
\text { resources }\end{array}$ & $\begin{array}{l}\text { impl; possi- } \\
\text { bly process } \\
\text { performance }\end{array}$ & derived & $\begin{array}{l}\text { GG2.GP3 } \\
\text { GG2.GP8 }\end{array}$ \\
\hline
\end{tabular}

$8 \quad$ Measures referring to other types of policies specific to a PA are included in the PA measures table.

9 Policies as used here refer to new and updated organizational policies that reflect the intent of CERT-RMM process areas goals and practices.

10 Organizations are able to select specific goals (SGs) and specific practices (SPs) from CERT-RMM that support their organizational resilience objectives. 


\begin{tabular}{|c|c|c|c|c|c|}
\hline ID & Measure & $\begin{array}{l}\text { Type of In- } \\
\text { formation }\end{array}$ & $\begin{array}{l}\text { Measure } \\
\text { Type }\end{array}$ & $\begin{array}{l}\text { Base or } \\
\text { Derived }\end{array}$ & $\begin{array}{l}\text { Applicable } \\
\text { GG.GP }\end{array}$ \\
\hline G-M22 & $\begin{array}{l}\text { percentage of process activities that do not } \\
\text { have the necessary methods, techniques, and } \\
\text { tools to support them }\end{array}$ & resources & impl & derived & $\begin{array}{l}\text { GG2.GP3 } \\
\text { GG2.GP8 }\end{array}$ \\
\hline G-M23 & $\begin{array}{l}\text { percentage of process tasks where responsibil- } \\
\text { ity and authority for performing them is not } \\
\text { assigned }\end{array}$ & $\begin{array}{l}\text { responsibili- } \\
\text { ties }\end{array}$ & impl & derived & $\begin{array}{l}\text { GG2.GP4 } \\
\text { GG2.GP8 }\end{array}$ \\
\hline G-M24 & $\begin{array}{l}\text { percentage of staff who have been assessed } \\
\text { to determine if training has been effective }{ }^{11} \\
\text { commensurate with their job responsibilities } \\
\text { (duplicated from OTA; effectiveness) }\end{array}$ & training & effectiveness & derived & $\begin{array}{l}\text { GG2.GP5 } \\
\text { GG2.GP8 }\end{array}$ \\
\hline G-M25 & $\begin{array}{l}\text { difference in planned versus actual designated } \\
\text { work products that are subject to configuration } \\
\text { control }\end{array}$ & $\begin{array}{l}\text { controlled } \\
\text { work prod- } \\
\text { ucts }\end{array}$ & impl & derived & $\begin{array}{l}\text { GG2.GP6 } \\
\text { GG2.GP8 }\end{array}$ \\
\hline G-M26 & $\begin{array}{l}\text { difference in planned versus actual stakehold- } \\
\text { ers involved in the process }\end{array}$ & stakeholders & impl & derived & $\begin{array}{l}\text { GG2.GP7 } \\
\text { GG2.GP8 }\end{array}$ \\
\hline G-M27 & $\begin{array}{l}\text { percentage of processes whose performance } \\
\text { against plan is measured }\end{array}$ & $\begin{array}{l}\text { process per- } \\
\text { formance }\end{array}$ & impl & derived & $\begin{array}{l}\text { GG2.GP8 } \\
\text { GG2.GP9 }\end{array}$ \\
\hline G-M28 & $\begin{array}{l}\text { difference in planned versus actual process } \\
\text { performance }\end{array}$ & $\begin{array}{l}\text { process per- } \\
\text { formance }\end{array}$ & impl & derived & $\begin{array}{l}\text { GG2.GP8 } \\
\text { GG2.GP9 }\end{array}$ \\
\hline G-M29 & $\begin{array}{l}\text { percentage of significant deviations from the } \\
\text { process plan without corrective actions }\end{array}$ & $\begin{array}{l}\text { process per- } \\
\text { formance; } \\
\text { plan devia- } \\
\text { tions }\end{array}$ & impl & derived & $\begin{array}{l}\text { GG2.GP8 } \\
\text { GG2.GP9 }\end{array}$ \\
\hline G-M30 & $\begin{array}{l}\text { percentage of significant deviations from the } \\
\text { process plan with corrective actions that are on } \\
\text { track per plan }\end{array}$ & $\begin{array}{l}\text { process per- } \\
\text { formance; } \\
\text { plan devia- } \\
\text { tions }\end{array}$ & impl & derived & $\begin{array}{l}\text { GG2.GP8 } \\
\text { GG2.GP9 }\end{array}$ \\
\hline G-M31 & $\begin{array}{l}\text { percentage of process problems (performance, } \\
\text { execution) without corrective actions }\end{array}$ & $\begin{array}{l}\text { process per- } \\
\text { formance; } \\
\text { process prob- } \\
\text { lems }\end{array}$ & impl & derived & $\begin{array}{l}\text { GG2.GP8 } \\
\text { GG2.GP9 }\end{array}$ \\
\hline G-M32 & $\begin{array}{l}\text { percentage of process problems with correc- } \\
\text { tive actions that are on track per plan }\end{array}$ & $\begin{array}{l}\text { process per- } \\
\text { formance; } \\
\text { process prob- } \\
\text { lems }\end{array}$ & impl & derived & $\begin{array}{l}\text { GG2.GP8 } \\
\text { GG2.GP9 }\end{array}$ \\
\hline G-M33 & $\begin{array}{l}\text { number of process risks referred to the risk } \\
\text { management process }\end{array}$ & risk & impl & $\begin{array}{l}\text { base of } \\
\text { type count }\end{array}$ & GG2.GP8 \\
\hline G-M34 & $\begin{array}{l}\text { number of asset risks referred to the risk man- } \\
\text { agement process (applicable to ADM, EC, } \\
\text { KIM, PM, TM) }\end{array}$ & risk & impl & $\begin{array}{l}\text { base of } \\
\text { type count }\end{array}$ & GG2.GP8 \\
\hline G-M35 & $\begin{array}{l}\text { number of process risks referred to the risk } \\
\text { management process for which corrective } \\
\text { action is pending (by risk rank) beyond thre- } \\
\text { shold (schedule) }\end{array}$ & risk & impl & $\begin{array}{l}\text { base of } \\
\text { type count }\end{array}$ & $\begin{array}{l}\text { GG2.GP8 } \\
\text { GG2.GP10 }\end{array}$ \\
\hline G-M36 & $\begin{array}{l}\text { extent to which resilience }{ }^{12} \text { is improved as a } \\
\text { result of taking action on CERT-RMM diagnos- } \\
\text { tic results }{ }^{13} \text { as measured by, for example, a } \\
\text { reduction in impact and consequences due to } \\
\text { a disruptive event such as a security incident }\end{array}$ & $\begin{array}{l}\text { resilience } \\
\text { improvement }\end{array}$ & effectiveness & derived & $\begin{array}{l}\text { GG2.GP8 } \\
\text { GG2.GP9 } \\
\text { GG2.GP10 }\end{array}$ \\
\hline
\end{tabular}

11 OTA:SG4.SP3 provides several approaches for assessing training effectiveness.

12 This measure could apply to all 26 process areas, a selected set of process areas, or a targeted area of resilience improvement (such as selected specific goals and practices within the model scope for the diagnosis).

13 Diagnostic results include the outcomes of CERT-RMM appraisals, Compass, or other forms of diagnosis. This measure could be stated as implementing CERT-RMM process areas, specific goals, and specific practices. 


\title{
Asset Definition and Management (ADM)
}

The purpose of Asset Definition and Management is to identify, document, and manage organizational assets during their life cycle to ensure sustained productivity to support organizational services.

\section{Summary of Specific Goals and Practices}

ADM:SG1 Establish Organizational Assets

\author{
ADM:SG1.SP1 Inventory Assets
}

ADM:SG1.SP2 Establish a Common Understanding

ADM:SG1.SP3 Establish Ownership and Custodianship

ADM:SG2 Establish the Relationship Between Assets and Services

ADM:SG2.SP1 Associate Assets with Services

ADM:SG2.SP2 Analyze Asset-Service Dependencies

ADM:SG3 Manage Assets

ADM:SG3.SP1 Identify Change Criteria

ADM:SG3.SP2 Maintain Changes to Assets and Inventory

\section{Measures}

\begin{tabular}{|l|l|l|l|l|l|}
\hline ID $^{14}$ & Measure & $\begin{array}{l}\text { Type of } \\
\text { Information }\end{array}$ & $\begin{array}{l}\text { Measure } \\
\text { Type }\end{array}$ & $\begin{array}{l}\text { Base or } \\
\text { Derived }\end{array}$ & $\begin{array}{l}\text { Applicable } \\
\text { SG.SP }\end{array}$ \\
\hline $\begin{array}{l}\text { ADM- } \\
\text { M1 }\end{array}$ & $\begin{array}{l}\text { percentage of assets } \\
\text { toried }\end{array}$ & $\begin{array}{l}\text { asset inven- } \\
\text { tory }\end{array}$ & impl & derived & ADM:SG1.SP1 \\
\hline $\begin{array}{l}\text { ADM- } \\
\text { M2 }\end{array}$ & $\begin{array}{l}\text { percentage of assets with/without a complete } \\
\text { asset profile }\end{array}$ & $\begin{array}{l}\text { asset inven- } \\
\text { tory }\end{array}$ & impl & derived & ADM:SG1.SP2 \\
\hline $\begin{array}{l}\text { ADM- } \\
\text { M3 }\end{array}$ & $\begin{array}{l}\text { percentage of assets with/without a desig- } \\
\text { nated owner }\end{array}$ & $\begin{array}{l}\text { asset inven- } \\
\text { tory }\end{array}$ & impl & derived & ADM:SG1.SP3 \\
\hline $\begin{array}{l}\text { ADM- } \\
\text { M4 }\end{array}$ & $\begin{array}{l}\text { percentage of assets with/without a desig- } \\
\text { nated custodian (if applicable) }\end{array}$ & $\begin{array}{l}\text { asset inven- } \\
\text { tory }\end{array}$ & impl & derived & ADM:SG1.SP3 \\
\hline $\begin{array}{l}\text { ADM- } \\
\text { M5 }\end{array}$ & $\begin{array}{l}\text { percentage of assets that have designated } \\
\text { owners but no custodians (if applicable) }\end{array}$ & $\begin{array}{l}\text { asset inven- } \\
\text { tory }\end{array}$ & impl & derived & ADM:SG1.SP3 \\
\hline $\begin{array}{l}\text { ADM- } \\
\text { M6 }\end{array}$ & $\begin{array}{l}\text { percentage of assets that have designated } \\
\text { custodians but no owners }\end{array}$ & $\begin{array}{l}\text { asset inven- } \\
\text { tory }\end{array}$ & impl & derived & ADM:SG1.SP3 \\
\hline $\begin{array}{l}\text { ADM- } \\
\text { M7 }\end{array}$ & $\begin{array}{l}\text { percentage of assets that have been invento- } \\
\text { ried, by service }\end{array}$ & $\begin{array}{l}\text { asset inven- } \\
\text { tory }\end{array}$ & impl & derived & ADM:SG2.SP1 \\
\hline $\begin{array}{l}\text { ADM- } \\
\text { M8 }\end{array}$ & $\begin{array}{l}\text { percentage of assets that are not associated } \\
\text { with one or more services }\end{array}$ & $\begin{array}{l}\text { asset inven- } \\
\text { tory }\end{array}$ & impl & derived & ADM:SG2.SP1 \\
\hline $\begin{array}{l}\text { ADM- } \\
\text { M9 }\end{array}$ & $\begin{array}{l}\text { elapsed time since the asset inventory was } \\
\text { reviewed }\end{array}$ & $\begin{array}{l}\text { asset inven- } \\
\text { tory }\end{array}$ & impl & $\begin{array}{l}\text { base of } \\
\text { type } \\
\text { schedule }\end{array}$ & $\begin{array}{l}\text { ADM:SG1.SP1 } \\
\text { ADM:SG3.SP1 }\end{array}$ \\
\hline $\begin{array}{l}\text { ADM- } \\
\text { M10 }\end{array}$ & $\begin{array}{l}\text { percentage of asset-service dependency } \\
\text { mitigation plans }\end{array}$ & $\begin{array}{l}\text { asset-service } \\
\text { dependen- } \\
\text { cies }\end{array}$ & impl & derived & ADM:SG2.SP2 \\
\hline
\end{tabular}

14 The ID value is assigned based on the order in which the measure appears in CERT-RMM v1.1. Measures have been reordered here by the type of information.

15 All references to assets and services in ADM and in all other PAs refer to high-value assets and high-value services. This qualifier applies throughout and is not included for ease of reading. 


\begin{tabular}{|l|l|l|l|l|l|}
\hline ID $^{14}$ & Measure & $\begin{array}{l}\text { Type of } \\
\text { Information }\end{array}$ & $\begin{array}{l}\text { Measure } \\
\text { Type }\end{array}$ & $\begin{array}{l}\text { Base or } \\
\text { Derived }\end{array}$ & $\begin{array}{l}\text { Applicable } \\
\text { SG.SP }\end{array}$ \\
\hline $\begin{array}{l}\text { ADM- } \\
\text { M11 }\end{array}$ & $\begin{array}{l}\text { percentage of asset-service dependency } \\
\text { conflicts with no mitigation plans }\end{array}$ & $\begin{array}{l}\text { asset-service } \\
\text { dependen- } \\
\text { cies }\end{array}$ & impl & derived & ADM:SG2.SP2 \\
\hline $\begin{array}{l}\text { ADM- } \\
\text { M12 }\end{array}$ & $\begin{array}{l}\text { number of discrepancies between the current } \\
\text { inventory and the previous inventory }\end{array}$ & $\begin{array}{l}\text { asset change } \\
\text { management }\end{array}$ & impl & $\begin{array}{l}\text { base of } \\
\text { type } \\
\text { count }\end{array}$ & ADM:SG3.SP1 \\
\hline $\begin{array}{l}\text { ADM- } \\
\text { M13 }\end{array}$ & $\begin{array}{l}\text { number of changes made to asset profiles in } \\
\text { the asset inventory }\end{array}$ & $\begin{array}{l}\text { asset change } \\
\text { management }\end{array}$ & impl & $\begin{array}{l}\text { base of } \\
\text { type } \\
\text { count }\end{array}$ & ADM:SG3.SP2 \\
\hline $\begin{array}{l}\text { ADM- } \\
\text { M14 }\end{array}$ & $\begin{array}{l}\text { number of changes to resilience require- } \\
\text { ments as a result of asset changes }\end{array}$ & $\begin{array}{l}\text { asset change } \\
\text { management }\end{array}$ & impl & $\begin{array}{l}\text { base of } \\
\text { type } \\
\text { count }\end{array}$ & ADM:SG3.SP2 \\
\hline $\begin{array}{l}\text { ADM- } \\
\text { M15 }\end{array}$ & $\begin{array}{l}\text { number of changes to service continuity } \\
\text { plans as a result of asset changes }\end{array}$ & $\begin{array}{l}\text { asset change } \\
\text { management }\end{array}$ & impl & $\begin{array}{l}\text { base of } \\
\text { type } \\
\text { count }\end{array}$ & ADM:SG3.SP2 \\
\hline
\end{tabular}

\section{Access Management (AM)}

The purpose of Access Management is to ensure that access granted to organizational assets is commensurate with their business and resilience requirements.

\section{Summary of Specific Goals and Practices}

AM:SG1 Manage and Control Access

AM:SG1.SP1 Enable Access

AM:SG1.SP2 Manage Changes to Access Privileges

AM:SG1.SP3 Periodically Review and Maintain Access Privileges

AM:SG1.SP4 Correct Inconsistencies

\section{Measures}

\begin{tabular}{|c|c|c|c|c|c|}
\hline ID & Measure & $\begin{array}{l}\text { Type of } \\
\text { Information }\end{array}$ & $\begin{array}{l}\text { Measure } \\
\text { Type }\end{array}$ & $\begin{array}{l}\text { Base or } \\
\text { Derived }\end{array}$ & $\begin{array}{l}\text { Applicable } \\
\text { SG.SP }\end{array}$ \\
\hline AM-M1 & $\begin{array}{l}\text { percentage of asset owners participating in } \\
\text { establishing and maintaining access privileg- } \\
\text { es for the assets that they own }\end{array}$ & $\begin{array}{l}\text { access } \\
\text { privileges }\end{array}$ & impl & derived & AM:SG1.SP1 \\
\hline AM-M2 & $\begin{array}{l}\text { percentage of access requests that adhere to } \\
\text { the access control policy }\end{array}$ & $\begin{array}{l}\text { access } \\
\text { policy }\end{array}$ & impl & derived & AM:SG1.SP1 \\
\hline AM-M3 & $\begin{array}{l}\text { percentage of access acknowledgement } \\
\text { forms that have been fully executed }\end{array}$ & $\begin{array}{l}\text { access } \\
\text { policy }\end{array}$ & impl & derived & AM:SG1.SP1 \\
\hline AM-M4 & $\begin{array}{l}\text { percentage of access requests denied (based } \\
\text { on policy) }\end{array}$ & $\begin{array}{l}\text { access } \\
\text { requests }\end{array}$ & impl & derived & AM:SG1.SP1 \\
\hline AM-M5 & $\begin{array}{l}\text { percentage of approved access requests } \\
\text { pending implementation beyond schedule }\end{array}$ & $\begin{array}{l}\text { access } \\
\text { requests }\end{array}$ & impl & derived & AM:SG1.SP1 \\
\hline AM-M6 & number of duplicate access requests & $\begin{array}{l}\text { access } \\
\text { requests }\end{array}$ & impl & $\begin{array}{l}\text { base of type } \\
\text { count }\end{array}$ & AM:SG1.SP1 \\
\hline AM-M7 & $\begin{array}{l}\text { percentage of unapproved access requests } \\
\text { that result in allowing access privileges (this } \\
\text { should be zero) }\end{array}$ & $\begin{array}{l}\text { access } \\
\text { requests }\end{array}$ & $\begin{array}{l}\text { effec- } \\
\text { tiveness }\end{array}$ & derived & AM:SG1.SP1 \\
\hline AM-M8 & $\begin{array}{l}\text { percentage of access requests that do not } \\
\text { reflect the requestor's role or job responsibili- } \\
\text { ties (inadequate, excessive) }\end{array}$ & $\begin{array}{l}\text { access } \\
\text { requests }\end{array}$ & $\begin{array}{l}\text { effec- } \\
\text { tiveness }\end{array}$ & derived & AM:SG1.SP1 \\
\hline
\end{tabular}




\begin{tabular}{|c|c|c|c|c|c|}
\hline ID & Measure & $\begin{array}{l}\text { Type of } \\
\text { Information }\end{array}$ & $\begin{array}{l}\text { Measure } \\
\text { Type }\end{array}$ & $\begin{array}{l}\text { Base or } \\
\text { Derived }\end{array}$ & $\begin{array}{l}\text { Applicable } \\
\text { SG.SP }\end{array}$ \\
\hline AM-M9 & $\begin{array}{l}\text { percentage of access privileges that are de- } \\
\text { termined to be excessive or inappropriate } \\
\text { based on the identity's role or job responsibili- } \\
\text { ties }\end{array}$ & $\begin{array}{l}\text { access } \\
\text { privileges }\end{array}$ & $\begin{array}{l}\text { effec- } \\
\text { tiveness }\end{array}$ & derived & AM:SG1.SP3 \\
\hline $\begin{array}{l}\text { AM- } \\
\text { M10 }\end{array}$ & $\begin{array}{l}\text { elapsed time since access privileges were } \\
\text { reviewed to ensure they reflect privileges } \\
\text { assigned by the asset owner }\end{array}$ & $\begin{array}{l}\text { access } \\
\text { privileges }\end{array}$ & impl & $\begin{array}{l}\text { base of type } \\
\text { schedule }\end{array}$ & AM:SG1.SP3 \\
\hline $\begin{array}{l}\text { AM- } \\
\text { M11 }\end{array}$ & rate of requests to change access privileges & $\begin{array}{l}\text { access } \\
\text { privileges }\end{array}$ & impl & derived & $\begin{array}{l}\text { AM:SG1.SP2 } \\
\text { AM:SG1.SP4 }\end{array}$ \\
\hline $\begin{array}{l}\text { AM- } \\
\text { M12 }\end{array}$ & $\begin{array}{l}\text { percentage of access privilege change re- } \\
\text { quests approved/denied }\end{array}$ & $\begin{array}{l}\text { access } \\
\text { privileges }\end{array}$ & impl & derived & AM:SG1.SP2 \\
\hline $\begin{array}{l}\text { AM- } \\
\text { M13 }\end{array}$ & $\begin{array}{l}\text { percentage of corrective actions to address } \\
\text { excessive or inappropriate levels of access } \\
\text { privileges pending beyond schedule }\end{array}$ & $\begin{array}{l}\text { access } \\
\text { privileges }\end{array}$ & impl & derived & AM:SG1.SP4 \\
\hline $\begin{array}{l}\text { AM- } \\
\text { M14 }\end{array}$ & $\begin{array}{l}\text { elapsed time from a change in access privi- } \\
\text { leges requiring deprovisioning to the actual } \\
\text { deprovisioning (mean, median) }\end{array}$ & $\begin{array}{l}\text { deprovision- } \\
\text { ing }\end{array}$ & $\begin{array}{l}\text { effec- } \\
\text { tiveness }\end{array}$ & derived & $\begin{array}{l}\text { AM:SG1.SP4 } \\
\text { ID:SG2.SP4 }\end{array}$ \\
\hline $\begin{array}{l}\text { AM- } \\
\text { M15 }\end{array}$ & $\begin{array}{l}\text { number of risks related to inappropriate or } \\
\text { excessive levels of access privileges that } \\
\text { have been referred to the risk management } \\
\text { process }\end{array}$ & $\begin{array}{l}\text { risk identifi- } \\
\text { cation }\end{array}$ & impl & $\begin{array}{l}\text { base of type } \\
\text { count }\end{array}$ & $\begin{array}{l}\text { AM:SG1.SP1 } \\
\text { AM:SG1.SP4 }\end{array}$ \\
\hline
\end{tabular}

\section{Communications (COMM)}

The purpose of Communications is to develop, deploy, and manage internal and external communications to support resilience activities and processes.

\section{Summary of Specific Goals and Practices}

COMM:SG1 Prepare for Resilience Communications

COMM:SG1.SP1 Identify Relevant Stakeholders

COMM:SG1.SP2 Identify Communications Requirements

COMM:SG1.SP3 Establish Communications Guidelines and Standards

COMM:SG2 Prepare for Communications Management

COMM:SG2.SP1 Establish a Resilience Communications Plan

COMM:SG2.SP2 Establish a Resilience Communications Program

COMM:SG2.SP3 Identify and Assign Plan Staff

COMM:SG3 Deliver Resilience Communications

COMM:SG3.SP1 Identify Communications Methods and Channels

COMM:SG3.SP2 Establish and Maintain Communications Infrastructure

COMM:SG4 Improve Communications

COMM:SG4.SP1 Assess Communications Effectiveness

COMM:SG4.SP2 Improve Communications 


\section{Measures}

\begin{tabular}{|c|c|c|c|c|c|}
\hline ID & Measure & $\begin{array}{l}\text { Type of } \\
\text { Information }\end{array}$ & $\begin{array}{l}\text { Measure } \\
\text { Type }\end{array}$ & $\begin{array}{l}\text { Base or } \\
\text { Derived }\end{array}$ & $\begin{array}{l}\text { Applicable } \\
\text { SG.SP }\end{array}$ \\
\hline $\begin{array}{l}\text { COMM- } \\
\text { M1 }\end{array}$ & $\begin{array}{l}\text { confidence factor }^{16} \text { that all stakeholders with } \\
\text { a vested interest or vital role in resilience } \\
\text { communications have been identified }\end{array}$ & $\begin{array}{l}\text { communications } \\
\text { stakeholders }\end{array}$ & impl & derived & $\begin{array}{l}\text { COMM:SG1. } \\
\text { SP1 }\end{array}$ \\
\hline $\begin{array}{l}\text { COMM- } \\
\text { M2 }\end{array}$ & $\begin{array}{l}\text { percentage of communications stakeholders } \\
\text { for which roles have/have not been defined }\end{array}$ & $\begin{array}{l}\text { communications } \\
\text { stakeholders }\end{array}$ & impl & derived & $\begin{array}{l}\text { COMM:SG1. } \\
\text { SP1 }\end{array}$ \\
\hline $\begin{array}{l}\text { COMM- } \\
\text { M3 }\end{array}$ & $\begin{array}{l}\text { percentage of communications stakeholders } \\
\text { for which stakeholder needs (types, fre- } \\
\text { quencies, and levels of communication by } \\
\text { specific circumstance) have/have not been } \\
\text { defined }\end{array}$ & $\begin{array}{l}\text { communications } \\
\text { stakeholders }\end{array}$ & impl & derived & $\begin{array}{l}\text { COMM:SG1. } \\
\text { SP1 }\end{array}$ \\
\hline $\begin{array}{l}\text { COMM- } \\
\text { M4 }\end{array}$ & $\begin{array}{l}\text { percentage of communications stakeholders } \\
\text { for which resilience communications and } \\
\text { requirements have/have not been defined }\end{array}$ & $\begin{array}{l}\text { communications } \\
\text { stakeholders; } \\
\text { communications } \\
\text { requirements }\end{array}$ & impl & derived & $\begin{array}{l}\text { COMM:SG1. } \\
\text { SP2 }\end{array}$ \\
\hline $\begin{array}{l}\text { COMM- } \\
\text { M5 }\end{array}$ & $\begin{array}{l}\text { percentage of resilience communications } \\
\text { requirements that cannot be met (by some } \\
\text { meaningful categorization such as missing, } \\
\text { inadequate, or untrained staff; missing or } \\
\text { inadequate tools, techniques, methods, } \\
\text { etc.- a.k.a. infrastructure) }\end{array}$ & $\begin{array}{l}\text { communications } \\
\text { requirements }\end{array}$ & impl & derived & $\begin{array}{l}\text { COMM:SG1. } \\
\text { SP2 } \\
\text { COMM:SG2. } \\
\text { SP3 } \\
\text { COMM:SG3. } \\
\text { SP2 }\end{array}$ \\
\hline $\begin{array}{l}\text { COMM- } \\
\text { M6 }\end{array}$ & $\begin{array}{l}\text { percentage of communications plan roles } \\
\text { not covered in job descriptions }\end{array}$ & $\begin{array}{l}\text { communications } \\
\text { staff }\end{array}$ & impl & derived & $\begin{array}{l}\text { COMM:SG2. } \\
\text { SP3 }\end{array}$ \\
\hline $\begin{array}{l}\text { COMM- } \\
\text { M7 }\end{array}$ & $\begin{array}{l}\text { percentage of stakeholders (by type) for } \\
\text { which communications methods and chan- } \\
\text { nels have/have not been identified }\end{array}$ & $\begin{array}{l}\text { communications } \\
\text { stakeholders; } \\
\text { communications } \\
\text { methods and } \\
\text { channels }\end{array}$ & impl & derived & $\begin{array}{l}\text { COMM:SG3. } \\
\text { SP1 }\end{array}$ \\
\hline $\begin{array}{l}\text { COMM- } \\
\text { M8 }\end{array}$ & $\begin{array}{l}\text { number of new communications methods } \\
\text { and channels }\end{array}$ & $\begin{array}{l}\text { communications } \\
\text { methods and } \\
\text { channels }\end{array}$ & impl & $\begin{array}{l}\text { base of } \\
\text { type count }\end{array}$ & $\begin{array}{l}\text { COMM:SG3. } \\
\text { SP1 }\end{array}$ \\
\hline $\begin{array}{l}\text { COMM- } \\
\text { M9 }\end{array}$ & $\begin{array}{l}\text { percentage of methods and channels with } \\
\text { sufficient infrastructure to support them }\end{array}$ & $\begin{array}{l}\text { communications } \\
\text { methods and } \\
\text { channels }\end{array}$ & impl & derived & $\begin{array}{l}\text { COMM:SG3. } \\
\text { SP1 }\end{array}$ \\
\hline $\begin{array}{l}\text { COMM- } \\
\text { M10 }\end{array}$ & $\begin{array}{l}\text { number of communications delivered by } \\
\text { event type, stakeholder type, method and } \\
\text { channel type (or other meaningful categori- } \\
\text { zation) }\end{array}$ & $\begin{array}{l}\text { communications } \\
\text { delivery }\end{array}$ & impl & $\begin{array}{l}\text { base of } \\
\text { type count }\end{array}$ & $\begin{array}{l}\text { COMM:SG4. } \\
\text { SP1 }\end{array}$ \\
\hline $\begin{array}{l}\text { COMM- } \\
\text { M11 }\end{array}$ & $\begin{array}{l}\text { percentage of communications methods } \\
\text { and channels operating within expected } \\
\text { tolerances (e.g., press release must be } \\
\text { issued within one hour of a significant } \\
\text { event) }\end{array}$ & $\begin{array}{l}\text { communications } \\
\text { delivery; com- } \\
\text { munications } \\
\text { methods and } \\
\text { channels }\end{array}$ & $\begin{array}{l}\text { effec- } \\
\text { tiveness }\end{array}$ & derived & $\begin{array}{l}\text { COMM:SG4. } \\
\text { SP1 }\end{array}$ \\
\hline $\begin{array}{l}\text { COMM- } \\
\text { M12 }\end{array}$ & $\begin{array}{l}\text { change (increase or decrease) in length of } \\
\text { time to commence communications by } \\
\text { event type }\end{array}$ & $\begin{array}{l}\text { communications } \\
\text { delivery }\end{array}$ & impl & derived & $\begin{array}{l}\text { COMM:SG4. } \\
\text { SP1 }\end{array}$ \\
\hline $\begin{array}{l}\text { COMM- } \\
\text { M13 }\end{array}$ & $\begin{array}{l}\text { percentage of stakeholders that do not } \\
\text { receive communications within expected } \\
\text { tolerances, by stakeholder type and by } \\
\text { event type }\end{array}$ & $\begin{array}{l}\text { communications } \\
\text { delivery; com- } \\
\text { munications } \\
\text { stakeholders }\end{array}$ & $\begin{array}{l}\text { effec- } \\
\text { tiveness }\end{array}$ & derived & $\begin{array}{l}\text { COMM:SG4. } \\
\text { SP1 }\end{array}$ \\
\hline
\end{tabular}

16 Refer to comparable measure and template in Measuring Operational Resilience Using the CERT Resilience Management Model [Allen 2010], section 4.1.1. 


\begin{tabular}{|c|c|c|c|c|c|}
\hline ID & Measure & $\begin{array}{l}\text { Type of } \\
\text { Information }\end{array}$ & $\begin{array}{l}\text { Measure } \\
\text { Type }\end{array}$ & $\begin{array}{l}\text { Base or } \\
\text { Derived }\end{array}$ & $\begin{array}{l}\text { Applicable } \\
\text { SG.SP }\end{array}$ \\
\hline $\begin{array}{l}\text { COMM- } \\
\text { M14 }\end{array}$ & $\begin{array}{l}\text { number of communications methods and } \\
\text { channels required to deliver the same or } \\
\text { similar messages }\end{array}$ & $\begin{array}{l}\text { communications } \\
\text { delivery; com- } \\
\text { munications } \\
\text { methods and } \\
\text { channels }\end{array}$ & impl & $\begin{array}{l}\text { base of } \\
\text { type count }\end{array}$ & $\begin{array}{l}\text { COMM:SG4. } \\
\text { SP1 }\end{array}$ \\
\hline $\begin{array}{l}\text { COMM- } \\
\text { M15 }\end{array}$ & $\begin{array}{l}\text { percentage of uptime or availability (down- } \\
\text { time) of preferred communications methods, } \\
\text { channels, and infrastructure }\end{array}$ & $\begin{array}{l}\text { communications } \\
\text { methods and } \\
\text { channels; com- } \\
\text { munications } \\
\text { infrastructure }\end{array}$ & $\begin{array}{l}\text { effec- } \\
\text { tiveness }\end{array}$ & derived & $\begin{array}{l}\text { COMM:SG4. } \\
\text { SP1 }\end{array}$ \\
\hline $\begin{array}{l}\text { COMM- } \\
\text { M16 }\end{array}$ & $\begin{array}{l}\text { number of recommendations for improve- } \\
\text { ment referred to the event, incident, service } \\
\text { continuity, and crisis management } \\
\text { processes }\end{array}$ & $\begin{array}{l}\text { process im- } \\
\text { provement }\end{array}$ & impl & $\begin{array}{l}\text { base of } \\
\text { type count }\end{array}$ & $\begin{array}{l}\text { COMM:SG4. } \\
\text { SP1 }\end{array}$ \\
\hline $\begin{array}{l}\text { COMM- } \\
\text { M17 }\end{array}$ & $\begin{array}{l}\text { percentage of communications deficiencies } \\
\text { and omissions for which corrective action is } \\
\text { pending beyond schedule }\end{array}$ & $\begin{array}{l}\text { communications } \\
\text { deficiencies }\end{array}$ & $\begin{array}{l}\text { effec- } \\
\text { tiveness }\end{array}$ & derived & $\begin{array}{l}\text { COMM:SG4. } \\
\text { SP2 }\end{array}$ \\
\hline $\begin{array}{l}\text { COMM- } \\
\text { M18 }\end{array}$ & $\begin{array}{l}\text { number of service continuity plans that } \\
\text { require updates as a result of communica- } \\
\text { tions deficiencies or omissions }\end{array}$ & $\begin{array}{l}\text { communications } \\
\text { deficiencies; } \\
\text { service continui- } \\
\text { ty plans }\end{array}$ & $\begin{array}{l}\text { effec- } \\
\text { tiveness }\end{array}$ & $\begin{array}{l}\text { base of } \\
\text { type count }\end{array}$ & $\begin{array}{l}\text { COMM:SG4. } \\
\text { SP2 }\end{array}$ \\
\hline $\begin{array}{l}\text { COMM- } \\
\text { M19 }\end{array}$ & $\begin{array}{l}\text { number of communications failures resulting } \\
\text { from lack of adherence to resilience com- } \\
\text { munications guidelines and standards }\end{array}$ & $\begin{array}{l}\text { communications } \\
\text { deficiencies; } \\
\text { communications } \\
\text { guidelines and } \\
\text { standards }\end{array}$ & $\begin{array}{l}\text { effec- } \\
\text { tiveness }\end{array}$ & $\begin{array}{l}\text { base of } \\
\text { type count }\end{array}$ & $\begin{array}{l}\text { COMM:SG1. } \\
\text { SP3 }\end{array}$ \\
\hline $\begin{array}{l}\text { COMM- } \\
\text { M20 }\end{array}$ & $\begin{array}{l}\text { percentage of resilience communications } \\
\text { objectives that are being achieved accord- } \\
\text { ing to plan }\end{array}$ & plan status & impl & derived & $\begin{array}{l}\text { COMM:SG2. } \\
\text { SP1 }\end{array}$ \\
\hline
\end{tabular}

\section{Compliance (COMP)}

The purpose of Compliance is to ensure awareness of and compliance with an established set of relevant internal and external guidelines, standards, practices, policies, regulations, and legislation, and other obligations (such as contracts and service level agreements) related to managing operational resilience.

\section{Summary of Specific Goals and Practices}

COMP:SG1 Prepare for Compliance Management

COMP:SG1.SP1 Establish a Compliance Plan

COMP:SG1.SP2 Establish a Compliance Program

COMP:SG1.SP3 Establish Compliance Guidelines and Standards

COMP:SG2 Establish Compliance Obligations

COMP:SG2.SP1 Identify Compliance Obligations

COMP:SG2.SP2 Analyze Obligations

COMP:SG2.SP3 Establish Ownership for Meeting Obligations

COMP:SG3 Demonstrate Satisfaction of Compliance Obligations

COMP:SG3.SP1 Collect and Validate Compliance Data

COMP:SG3.SP2 Demonstrate the Extent of Compliance Obligation Satisfaction 
COMP:SG3.SP3 Remediate Areas of Non-Compliance

COMP:SG4 Monitor Compliance Activities

COMP:SG4.SP1 Evaluate Compliance Activities

\section{Measures}

\begin{tabular}{|c|c|c|c|c|c|}
\hline ID & Measure & $\begin{array}{l}\text { Type of } \\
\text { Information }\end{array}$ & $\begin{array}{l}\text { Measure } \\
\text { Type }\end{array}$ & $\begin{array}{l}\text { Base or } \\
\text { Derived }\end{array}$ & $\begin{array}{l}\text { Applicable } \\
\text { SG.SP }\end{array}$ \\
\hline $\begin{array}{l}\text { COMP- } \\
\text { M1 }\end{array}$ & $\begin{array}{l}\text { time expended to gather, organize, analyze, } \\
\text { and report data for compliance obligations }{ }^{17}\end{array}$ & $\begin{array}{l}\text { compliance } \\
\text { data }\end{array}$ & impl & derived & $\begin{array}{l}\text { COMP:SG2. } \\
\text { SP1 }\end{array}$ \\
\hline $\begin{array}{l}\text { COMP- } \\
\text { M2 }\end{array}$ & $\begin{array}{l}\text { percentage of compliance obligation data } \\
\text { collection activities that are/are not automated }\end{array}$ & $\begin{array}{l}\text { compliance } \\
\text { data }\end{array}$ & impl & derived & $\begin{array}{l}\text { COMP:SG1. } \\
\text { SP2 } \\
\text { COMP:SG1. } \\
\text { SP3 }\end{array}$ \\
\hline $\begin{array}{l}\text { COMP- } \\
\text { M3 }\end{array}$ & $\begin{array}{l}\text { number of compliance obligations (may re- } \\
\text { quire some prioritization of obligations such } \\
\text { as high, medium, low) }\end{array}$ & $\begin{array}{l}\text { obligation } \\
\text { inventory }\end{array}$ & impl & $\begin{array}{l}\text { base of type } \\
\text { count }\end{array}$ & $\begin{array}{l}\text { COMP:SG2. } \\
\text { SP1 }\end{array}$ \\
\hline $\begin{array}{l}\text { COMP- } \\
\text { M4 }\end{array}$ & $\begin{array}{l}\text { percentage of compliance obligations that } \\
\text { have been inventoried }\end{array}$ & $\begin{array}{l}\text { obligation } \\
\text { inventory }\end{array}$ & impl & derived & $\begin{array}{l}\text { COMP:SG2. } \\
\text { SP1 }\end{array}$ \\
\hline $\begin{array}{l}\text { COMP- } \\
\text { M5 }\end{array}$ & $\begin{array}{l}\text { percentage of compliance obligations } \\
\text { with/without a designated owner (organiza- } \\
\text { tional unit, line of business) }\end{array}$ & $\begin{array}{l}\text { obligation } \\
\text { inventory }\end{array}$ & impl & derived & $\begin{array}{l}\text { COMP:SG2. } \\
\text { SP1 } \\
\text { COMP:SG2. } \\
\text { SP3 }\end{array}$ \\
\hline $\begin{array}{l}\text { COMP- } \\
\text { M6 }\end{array}$ & $\begin{array}{l}\text { number of external entities with agreements } \\
\text { to meet compliance obligations }\end{array}$ & $\begin{array}{l}\text { obligation } \\
\text { inventory }\end{array}$ & impl & $\begin{array}{l}\text { base of type } \\
\text { count }\end{array}$ & $\begin{array}{l}\text { COMP:SG1. } \\
\text { SP3 } \\
\text { COMP:SG2. } \\
\text { SP1 } \\
\text { EXD:SG1.SP } \\
1\end{array}$ \\
\hline $\begin{array}{l}\text { COMP- } \\
\text { M7 }\end{array}$ & $\begin{array}{l}\text { percentage of compliance obligations that rely } \\
\text { upon external dependencies }\end{array}$ & $\begin{array}{l}\text { obligation } \\
\text { inventory }\end{array}$ & impl & derived & $\begin{array}{l}\text { COMP:SG2. } \\
\text { SP1 } \\
\text { EXD:SG1.SP } \\
1\end{array}$ \\
\hline $\begin{array}{l}\text { COMP- } \\
\text { M8 }\end{array}$ & $\begin{array}{l}\text { percentage of compliance obligations that rely } \\
\text { upon external entities }\end{array}$ & $\begin{array}{l}\text { obligation } \\
\text { inventory }\end{array}$ & impl & derived & $\begin{array}{l}\text { COMP:SG2. } \\
\text { SP1 } \\
\text { EXD:SG1.SP } \\
1\end{array}$ \\
\hline $\begin{array}{l}\text { COMP- } \\
\text { M9 }\end{array}$ & $\begin{array}{l}\text { percentage of compliance obligations that are } \\
\text { not met }\end{array}$ & $\begin{array}{l}\text { obligation } \\
\text { satisfaction }\end{array}$ & impl & derived & $\begin{array}{l}\text { COMP:SG3. } \\
\text { SP2 }\end{array}$ \\
\hline $\begin{array}{l}\text { COMP- } \\
\text { M10 }\end{array}$ & $\begin{array}{l}\text { percentage of compliance obligations not met } \\
\text { by deadline }\end{array}$ & $\begin{array}{l}\text { obligation } \\
\text { satisfaction }\end{array}$ & impl & derived & $\begin{array}{l}\text { COMP:SG3. } \\
\text { SP2 }\end{array}$ \\
\hline $\begin{array}{l}\text { COMP- } \\
\text { M11 }\end{array}$ & $\begin{array}{l}\text { percentage of compliance activities that do } \\
\text { not meet standards and guidelines }\end{array}$ & $\begin{array}{l}\text { obligation } \\
\text { satisfaction }\end{array}$ & impl & derived & $\begin{array}{l}\text { COMP:SG3. } \\
\text { SP2 }\end{array}$ \\
\hline $\begin{array}{l}\text { COMP- } \\
\text { M12 }\end{array}$ & $\begin{array}{l}\text { percentage of controls required solely to meet } \\
\text { compliance obligations }\end{array}$ & $\begin{array}{l}\text { obligation } \\
\text { satisfaction }\end{array}$ & impl & derived & $\begin{array}{l}\text { COMP:SG4. } \\
\text { SP1 }\end{array}$ \\
\hline $\begin{array}{l}\text { COMP- } \\
\text { M13 }\end{array}$ & $\begin{array}{l}\text { percentage of service continuity guidelines } \\
\text { and standards that are more/less stringent } \\
\text { than required to meet compliance obligations }\end{array}$ & $\begin{array}{l}\text { obligation } \\
\text { satisfaction }\end{array}$ & impl & derived & $\begin{array}{l}\text { SC:SG1.SP1 } \\
\text { SC:SG1.SP2 }\end{array}$ \\
\hline
\end{tabular}

17 Any reference to "compliance obligations" includes "(by category, by source)" as part of the definition of the measure. It is omitted from measures for ease of reading. 


\begin{tabular}{|c|c|c|c|c|c|}
\hline $\begin{array}{l}\text { COMP- } \\
\text { M14 }\end{array}$ & $\begin{array}{l}\text { number of compliance risks (exceptions, non- } \\
\text { compliance, remediation) referred to key } \\
\text { stakeholders (the risk management process, } \\
\text { the organization's governance process, etc.) }\end{array}$ & $\begin{array}{l}\text { obligation } \\
\text { remediation }\end{array}$ & $\begin{array}{l}\text { impl; risk } \\
\text { identifi- } \\
\text { cation }\end{array}$ & $\begin{array}{l}\text { base of type } \\
\text { count }\end{array}$ & $\begin{array}{l}\text { COMP:SG1. } \\
\text { SP2 } \\
\text { COMP:SG3. } \\
\text { SP2 } \\
\text { COMP:SG3. } \\
\text { SP3 }\end{array}$ \\
\hline $\begin{array}{l}\text { COMP- } \\
\text { M15 }\end{array}$ & $\begin{array}{l}\text { percentage of compliance obligation violations } \\
\text { requiring corrective action for which such } \\
\text { action has not been taken as scheduled }\end{array}$ & $\begin{array}{l}\text { obligation } \\
\text { remediation }\end{array}$ & impl & derived & $\begin{array}{l}\text { COMP:SG1. } \\
\text { SP2 }\end{array}$ \\
\hline $\begin{array}{l}\text { COMP- } \\
\text { M16 }\end{array}$ & $\begin{array}{l}\text { percentage of compliance obligations that are } \\
\text { conflicting (could also include duplicates, } \\
\text { redundancies, and overlaps, but conflicts are } \\
\text { likely of greatest interest) }\end{array}$ & $\begin{array}{l}\text { obligation } \\
\text { remediation }\end{array}$ & impl & derived & $\begin{array}{l}\text { COMP:SG2. } \\
\text { SP2 } \\
\text { (COMP:SG1 } \\
\text { SP2) }\end{array}$ \\
\hline $\begin{array}{l}\text { COMP- } \\
\text { M17 }\end{array}$ & $\begin{array}{l}\text { percentage of compliance obligations requir- } \\
\text { ing remediation for which the remediation } \\
\text { action results in the obligation being met }\end{array}$ & $\begin{array}{l}\text { obligation } \\
\text { remediation }\end{array}$ & impl & derived & $\begin{array}{l}\text { COMP:SG3. } \\
\text { SP3 }\end{array}$ \\
\hline $\begin{array}{l}\text { COMP- } \\
\text { M18 }\end{array}$ & cost to satisfy compliance obligations & $\begin{array}{l}\text { cost of } \\
\text { compliance }\end{array}$ & impl & $\begin{array}{l}\text { base of type } \\
\text { cost }\end{array}$ & $\begin{array}{l}\text { COMP:SG4. } \\
\text { SP1 }\end{array}$ \\
\hline $\begin{array}{l}\text { COMP- } \\
\text { M19 }\end{array}$ & $\begin{array}{l}\text { costs of non-compliance including: } \\
\text { amount of fines and penalties levied for non- } \\
\text { reporting } \\
\text { amount of fines and penalties levied for non- } \\
\text { compliance }\end{array}$ & $\begin{array}{l}\text { cost of } \\
\text { compliance }\end{array}$ & impl & $\begin{array}{l}\text { base of type } \\
\text { cost }\end{array}$ & $\begin{array}{l}\text { COMP:SG3. } \\
\text { SP2 } \\
\text { COMP:SG2. } \\
\text { SP1 }\end{array}$ \\
\hline $\begin{array}{l}\text { COMP- } \\
\text { M20 }\end{array}$ & $\begin{array}{l}\text { number of deficiencies in the compliance } \\
\text { process that directly resulted in compliance } \\
\text { obligations not being met }\end{array}$ & $\begin{array}{l}\text { compliance } \\
\text { process }\end{array}$ & $\begin{array}{l}\text { effec- } \\
\text { tiveness }\end{array}$ & $\begin{array}{l}\text { base of type } \\
\text { defect }\end{array}$ & $\begin{array}{l}\text { COMP:SG4. } \\
\text { SP1 }\end{array}$ \\
\hline $\begin{array}{l}\text { COMP- } \\
\text { M21 }\end{array}$ & $\begin{array}{l}\text { number of deficiencies in internal controls that } \\
\text { directly resulted in compliance obligations not } \\
\text { being met }\end{array}$ & $\begin{array}{l}\text { compliance } \\
\text { obligations; } \\
\text { internal } \\
\text { controls }\end{array}$ & $\begin{array}{l}\text { effec- } \\
\text { tiveness }\end{array}$ & $\begin{array}{l}\text { base of type } \\
\text { defect }\end{array}$ & $\begin{array}{l}\text { COMP:SG4. } \\
\text { SP1 }\end{array}$ \\
\hline $\begin{array}{l}\text { COMP- } \\
\text { M22 }\end{array}$ & $\begin{array}{l}\text { number of errors in the compliance process } \\
\text { caused by inaccurate or unavailable data }\end{array}$ & $\begin{array}{l}\text { compliance } \\
\text { process; } \\
\text { compliance } \\
\text { data }\end{array}$ & $\begin{array}{l}\text { effec- } \\
\text { tiveness }\end{array}$ & $\begin{array}{l}\text { base of type } \\
\text { defect }\end{array}$ & $\begin{array}{l}\text { COMP:SG3. } \\
\text { SP1 }\end{array}$ \\
\hline
\end{tabular}

\section{Controls Management (CTRL)}

The purpose of Controls Management is to establish, monitor, analyze, and manage an internal control system that ensures the effectiveness and efficiency of operations through assuring mission success of high-value services and the assets that support them.

\section{Summary of Specific Goals and Practices}

CTRL:SG1 Establish Control Objectives

CTRL:SG1.SP1 Define Control Objectives

CTRL:SG2 Establish Controls

CTRL:SG2.SP1 Define Controls

CTRL:SG3 Analyze Controls

CTRL:SG3.SP1 Analyze Controls

CTRL:SG4 Assess Control Effectiveness

CTRL:SG4.SP1 Assess Controls 


\section{Measures}

\begin{tabular}{|c|c|c|c|c|c|}
\hline ID & Measure & $\begin{array}{l}\text { Type of } \\
\text { Information }\end{array}$ & $\begin{array}{l}\text { Measure } \\
\text { Type }\end{array}$ & $\begin{array}{l}\text { Base or } \\
\text { Derived }\end{array}$ & $\begin{array}{l}\text { Applicable } \\
\text { SG.SP }\end{array}$ \\
\hline $\begin{array}{l}\text { CTRL- } \\
\text { M1 }\end{array}$ & $\begin{array}{l}\text { confidence factor }{ }^{18} \text { that control objectives } \\
\text { from all relevant management directives and } \\
\text { guidelines have been identified } \\
\text { at the enterprise level } \\
\text { at the service level (perhaps by service type) } \\
\text { at the asset level (perhaps by asset type) }\end{array}$ & $\begin{array}{l}\text { control ob- } \\
\text { jectives }\end{array}$ & $\begin{array}{l}\text { effec- } \\
\text { tiveness }\end{array}$ & derived & CTRL:SG1.SP1 \\
\hline $\begin{array}{l}\text { CTRL- } \\
\text { M2 }\end{array}$ & $\begin{array}{l}\text { percentage of control objectives that have } \\
\text { been prioritized (should be 100\%) }\end{array}$ & $\begin{array}{l}\text { control ob- } \\
\text { jectives }\end{array}$ & impl & derived & CTRL:SG1.SP1 \\
\hline $\begin{array}{l}\text { CTRL- } \\
\text { M3 }\end{array}$ & $\begin{array}{l}\text { percentage of enterprise-level controls for } \\
\text { which responsibility has been confirmed or } \\
\text { assigned }^{19}\end{array}$ & $\begin{array}{l}\text { enterprise } \\
\text { controls }\end{array}$ & impl & derived & CTRL:SG2.SP1 \\
\hline $\begin{array}{l}\text { CTRL- } \\
\text { M4 }\end{array}$ & $\begin{array}{l}\text { percentage of enterprise-level controls that do } \\
\text { not map to one or more control objectives }\end{array}$ & $\begin{array}{l}\text { enterprise } \\
\text { controls }\end{array}$ & impl & derived & CTRL:SG2.SP1 \\
\hline $\begin{array}{l}\text { CTRL- } \\
\text { M5 }\end{array}$ & $\begin{array}{l}\text { percentage of service-level controls for which } \\
\text { responsibility has been confirmed or assigned }\end{array}$ & $\begin{array}{l}\text { service } \\
\text { controls }\end{array}$ & impl & derived & CTRL:SG2.SP1 \\
\hline $\begin{array}{l}\text { CTRL- } \\
\text { M6 }\end{array}$ & $\begin{array}{l}\text { percentage of service-level controls that do } \\
\text { not map to one or more control objectives }\end{array}$ & $\begin{array}{l}\text { service } \\
\text { controls }\end{array}$ & impl & derived & CTRL:SG2.SP1 \\
\hline $\begin{array}{l}\text { CTRL- } \\
\text { M7 }\end{array}$ & $\begin{array}{l}\text { percentage of asset-level controls for which } \\
\text { responsibility has been confirmed or assigned }\end{array}$ & $\begin{array}{l}\text { asset con- } \\
\text { trols }\end{array}$ & impl & derived & CTRL:SG2.SP1 \\
\hline $\begin{array}{l}\text { CTRL- } \\
\text { M8 }\end{array}$ & $\begin{array}{l}\text { percentage of asset-level controls that do not } \\
\text { map to one or more control objectives }\end{array}$ & $\begin{array}{l}\text { asset con- } \\
\text { trols }\end{array}$ & impl & derived & CTRL:SG2.SP1 \\
\hline $\begin{array}{l}\text { CTRL- } \\
\text { M9 }\end{array}$ & $\begin{array}{l}\text { percentage of control objectives that are fully } \\
\text { satisfied by existing controls } \\
\text { at the enterprise level } \\
\text { at the service level (perhaps by service type) } \\
\text { at the asset level (perhaps by asset type) }\end{array}$ & $\begin{array}{l}\text { control ob- } \\
\text { jective satis- } \\
\text { faction }\end{array}$ & Impl & derived & CTRL:SG3.SP1 ${ }^{20}$ \\
\hline $\begin{array}{l}\text { CTRL- } \\
\text { M10 }\end{array}$ & $\begin{array}{l}\text { percentage of controls that satisfy multiple } \\
\text { control objectives (and mean, median number } \\
\text { of control objectives satisfied) }\end{array}$ & $\begin{array}{l}\text { control ob- } \\
\text { jective satis- } \\
\text { faction }\end{array}$ & Impl & derived & CTRL:SG3.SP1 \\
\hline $\begin{array}{l}\text { CTRL- } \\
\text { M11 }\end{array}$ & $\begin{array}{l}\text { percentage of controls that require updates to } \\
\text { address gaps }{ }^{21} \text { (perhaps by control objective) }\end{array}$ & $\begin{array}{l}\text { control ob- } \\
\text { jective satis- } \\
\text { faction; } \\
\text { control gaps }\end{array}$ & Impl & derived & CTRL:SG3.SP1 \\
\hline $\begin{array}{l}\text { CTRL- } \\
\text { M12 }\end{array}$ & $\begin{array}{l}\text { percentage of control objectives that are af- } \\
\text { fected by updated controls }\end{array}$ & $\begin{array}{l}\text { control ob- } \\
\text { jective satis- } \\
\text { faction; } \\
\text { control } \\
\text { changes }\end{array}$ & Impl & derived & CTRL:SG3.SP1 \\
\hline
\end{tabular}

18 Refer to comparable measure and template in Measuring Operational Resilience Using the CERT Resilience Management Model [Allen 2010], section 4.1.1.

19 Confirmation applies to existing and updated controls; assignment is required for new controls.

20 CTRL:SG3 establishes a baseline analysis of the extent to which existing controls and proposed new controls cover and achieve control objectives for the resilience of services and supporting assets. CTRL:SG4 uses this established baseline as the foundation for periodically assessing the extent to which controls continue to achieve control objectives and the extent to which control objectives continue to meet resilience requirements.

21 Where control objectives are not adequately satisfied by existing controls 


\begin{tabular}{|c|c|c|c|c|c|}
\hline ID & Measure & $\begin{array}{l}\text { Type of } \\
\text { Information }\end{array}$ & $\begin{array}{l}\text { Measure } \\
\text { Type }\end{array}$ & $\begin{array}{l}\text { Base or } \\
\text { Derived }\end{array}$ & $\begin{array}{l}\text { Applicable } \\
\text { SG.SP }\end{array}$ \\
\hline $\begin{array}{l}\text { CTRL- } \\
\text { M13 }\end{array}$ & $\begin{array}{l}\text { number of proposed new controls that are } \\
\text { required to address gaps (perhaps by control } \\
\text { objective) }\end{array}$ & $\begin{array}{l}\text { control ob- } \\
\text { jective satis- } \\
\text { faction; } \\
\text { control gaps }\end{array}$ & Impl & $\begin{array}{l}\text { base of } \\
\text { type } \\
\text { count }\end{array}$ & CTRL:SG3.SP1 \\
\hline $\begin{array}{l}\text { CTRL- } \\
\text { M14 }\end{array}$ & $\begin{array}{l}\text { percentage of control objectives that are af- } \\
\text { fected by proposed new controls }\end{array}$ & $\begin{array}{l}\text { control ob- } \\
\text { jective satis- } \\
\text { faction; } \\
\text { control } \\
\text { changes }\end{array}$ & Impl & derived & CTRL:SG3.SP1 \\
\hline $\begin{array}{l}\text { CTRL- } \\
\text { M15 }\end{array}$ & percentage of controls that are redundant & $\begin{array}{l}\text { control re- } \\
\text { dundancy }\end{array}$ & Impl & derived & CTRL:SG3.SP1 \\
\hline $\begin{array}{l}\text { CTRL- } \\
\text { M16 }\end{array}$ & $\begin{array}{l}\text { percentage of control objectives that are af- } \\
\text { fected by redundant controls }\end{array}$ & $\begin{array}{l}\text { control ob- } \\
\text { jectives; } \\
\text { control re- } \\
\text { dundancy }\end{array}$ & Impl & derived & CTRL:SG3.SP1 \\
\hline $\begin{array}{l}\text { CTRL- } \\
\text { M17 }\end{array}$ & $\begin{array}{l}\text { percentage of controls that are conflicting } \\
\text { (enterprise, service, asset) }\end{array}$ & $\begin{array}{l}\text { control con- } \\
\text { flicts }\end{array}$ & Impl & derived & CTRL:SG3.SP1 \\
\hline $\begin{array}{l}\text { CTRL- } \\
\text { M18 }\end{array}$ & $\begin{array}{l}\text { percentage of control objectives that are af- } \\
\text { fected by conflicting controls }\end{array}$ & $\begin{array}{l}\text { control ob- } \\
\text { jectives; } \\
\text { control con- } \\
\text { flicts }\end{array}$ & Impl & derived & CTRL:SG3.SP1 \\
\hline $\begin{array}{l}\text { CTRL- } \\
\text { M19 }\end{array}$ & $\begin{array}{l}\text { percentage of control issues that are resolved } \\
\text { in the required timeframe: } \\
\text { gaps resulting from unsatisfied control objec- } \\
\text { tives } \\
\text { redundant controls } \\
\text { conflicting controls }\end{array}$ & $\begin{array}{l}\text { control is- } \\
\text { sues; con- } \\
\text { trol changes }\end{array}$ & impl & derived & CTRL:SG3.SP1 \\
\hline $\begin{array}{l}\text { CTRL- } \\
\text { M20 }\end{array}$ & $\begin{array}{l}\text { for issues that are not resolved, number of } \\
\text { new/updated risks }{ }^{22} \text { (by risk rank) resulting } \\
\text { from } \\
\text { unsatisfied control objectives } \\
\text { unaddressed redundant controls } \\
\text { unaddressed conflicting controls }\end{array}$ & $\begin{array}{l}\text { control is- } \\
\text { sues; risk } \\
\text { identification }\end{array}$ & impl & $\begin{array}{l}\text { base of } \\
\text { type } \\
\text { count }\end{array}$ & CTRL:SG3.SP1 \\
\hline $\begin{array}{l}\text { CTRL- } \\
\text { M21 }\end{array}$ & $\begin{array}{l}\text { time and resources expended to conduct an } \\
\text { analysis of controls (establish the baseline) }\end{array}$ & $\begin{array}{l}\text { controls } \\
\text { analysis }\end{array}$ & impl & derived & CTRL:SG3.SP1 \\
\hline $\begin{array}{l}\text { CTRL- } \\
\text { M22 }\end{array}$ & $\begin{array}{l}\text { time and resources expended to conduct an } \\
\text { assessment of controls (periodic) }\end{array}$ & $\begin{array}{l}\text { controls } \\
\text { assessment }\end{array}$ & impl & derived & CTRL:SG4.SP1 \\
\hline $\begin{array}{l}\text { CTRL- } \\
\text { M23 }\end{array}$ & $\begin{array}{l}\text { number of problem areas resulting from the } \\
\text { assessment of controls (perhaps by control } \\
\text { objective) }\end{array}$ & $\begin{array}{l}\text { controls } \\
\text { assessment }\end{array}$ & impl & $\begin{array}{l}\text { base of } \\
\text { type } \\
\text { count }\end{array}$ & CTRL:SG4.SP1 \\
\hline $\begin{array}{l}\text { CTRL- } \\
\text { M24 }\end{array}$ & $\begin{array}{l}\text { number of problem areas escalated to higher } \\
\text { level managers for review }\end{array}$ & $\begin{array}{l}\text { controls } \\
\text { assessment; } \\
\text { control is- } \\
\text { sues }\end{array}$ & impl & $\begin{array}{l}\text { base of } \\
\text { type } \\
\text { count }\end{array}$ & CTRL:SG4.SP1 \\
\hline $\begin{array}{l}\text { CTRL- } \\
\text { M25 }\end{array}$ & $\begin{array}{l}\text { percentage of control objectives requiring } \\
\text { remediation plans }\end{array}$ & $\begin{array}{l}\text { control ob- } \\
\text { jectives }\end{array}$ & impl & derived & CTRL:SG4.SP1 \\
\hline $\begin{array}{l}\text { CTRL- } \\
\text { M26 }\end{array}$ & $\begin{array}{l}\text { for controls that can be automated, percen- } \\
\text { tage of controls that have been fully auto- } \\
\text { mated }\end{array}$ & $\begin{array}{l}\text { control au- } \\
\text { tomation }\end{array}$ & impl & derived & CTRL:SG4.SP1 \\
\hline
\end{tabular}

22 Risks result where the priority of a control objective and any resulting control gaps do not warrant further investment in updated or new controls. 


\begin{tabular}{|c|c|c|c|c|c|}
\hline ID & Measure & $\begin{array}{l}\text { Type of } \\
\text { Information }\end{array}$ & $\begin{array}{l}\text { Measure } \\
\text { Type }\end{array}$ & $\begin{array}{l}\text { Base or } \\
\text { Derived }\end{array}$ & $\begin{array}{l}\text { Applicable } \\
\text { SG.SP }\end{array}$ \\
\hline $\begin{array}{l}\text { CTRL- } \\
\text { M27 }\end{array}$ & $\begin{array}{l}\text { percentage of problem areas }{ }^{23} \text { that are/are } \\
\text { not resolved within threshold (as scheduled): } \\
\text { gaps resulting from unsatisfied control objec- } \\
\text { tives } \\
\text { redundant controls } \\
\text { conflicting controls }\end{array}$ & $\begin{array}{l}\text { control is- } \\
\text { sues; con- } \\
\text { trol changes }\end{array}$ & impl & derived & CTRL:SG4.SP1 \\
\hline $\begin{array}{l}\text { CTRL- } \\
\text { M28 }\end{array}$ & percentage reduction in number of controls & $\begin{array}{l}\text { control } \\
\text { changes }\end{array}$ & $\begin{array}{l}\text { impl; } \\
\text { possibly } \\
\text { effec- } \\
\text { tiveness }\end{array}$ & derived & CTRL:SG4.SP1 \\
\hline $\begin{array}{l}\text { CTRL- } \\
\text { M29 }\end{array}$ & $\begin{array}{l}\text { number of risks resulting from unresolved } \\
\text { problems in the internal control system that } \\
\text { are referred to the risk management process }\end{array}$ & $\begin{array}{l}\text { control is- } \\
\text { sues; risk } \\
\text { identification }\end{array}$ & impl & $\begin{array}{l}\text { base of } \\
\text { type } \\
\text { count }\end{array}$ & CTRL:SG4.SP1 \\
\hline $\begin{array}{l}\text { CTRL- } \\
\text { M30 }\end{array}$ & $\begin{array}{l}\text { number of updates to service continuity plans } \\
\text { that result from changes to the internal control } \\
\text { system }\end{array}$ & $\begin{array}{l}\text { service } \\
\text { continuity } \\
\text { plans; con- } \\
\text { trol changes }\end{array}$ & impl & $\begin{array}{l}\text { base of } \\
\text { type } \\
\text { count }\end{array}$ & CTRL:SG4.SP1 \\
\hline
\end{tabular}

\section{Environmental Control (EC)}

The purpose of Environmental Control is to establish and manage an appropriate level of physical, environmental, and geographical controls to support the resilient operations of services in organizational facilities.

\section{Summary of Specific Goals and Practices}

EC:SG1 Establish and Prioritize Facility Assets

EC:SG1.SP1 Prioritize Facility Assets

EC:SG1.SP2 Establish Resilience-Focused Facility Assets

EC:SG2 Protect Facility Assets

EC:SG2.SP1 Assign Resilience Requirements to Facility Assets

EC:SG2.SP2 Establish and Implement Controls

EC:SG3 Manage Facility Asset Risk

EC:SG3.SP1 Identify and Assess Facility Asset Risk

EC:SG3.SP2 Mitigate Facility Risks

EC:SG4 Control Operational Environment

EC:SG4.SP1 Perform Facility Sustainability Planning

EC:SG4.SP2 Maintain Environmental Conditions

EC:SG4.SP3 Manage Dependencies on Public Services

EC:SG4.SP4 Manage Dependencies on Public Infrastructure

EC:SG4.SP5 Plan for Facility Retirement

23 May want to limit this measure to those problem areas that require remediation plans. 
Measures

\begin{tabular}{|c|c|c|c|c|c|}
\hline ID & Measure & $\begin{array}{l}\text { Type of } \\
\text { Information }\end{array}$ & $\begin{array}{l}\text { Measure } \\
\text { Type }\end{array}$ & $\begin{array}{l}\text { Base or } \\
\text { Derived }\end{array}$ & $\begin{array}{l}\text { Applicable } \\
\text { SG.SP }\end{array}$ \\
\hline EC-M1 & $\begin{array}{l}\text { percentage of facility assets that have been } \\
\text { inventoried }\end{array}$ & asset inventory & impl & derived & $\begin{array}{l}\text { ADM:SG1.S } \\
\text { P1 }\end{array}$ \\
\hline EC-M2 & $\begin{array}{l}\text { percentage of facility assets with/without a } \\
\text { complete asset profile (such as no stated } \\
\text { resilience requirements) }\end{array}$ & asset inventory & impl & derived & $\begin{array}{l}\text { ADM:SG1.S } \\
\text { P2 } \\
\text { EC:SG2.SP1 }\end{array}$ \\
\hline EC-M3 & $\begin{array}{l}\text { percentage of facility assets with/without a } \\
\text { designated owner }\end{array}$ & asset inventory & impl & derived & $\begin{array}{l}\text { ADM:SG1.S } \\
\text { P3 }\end{array}$ \\
\hline EC-M4 & $\begin{array}{l}\text { percentage of facility assets with/without a } \\
\text { designated custodian (if applicable) }\end{array}$ & asset inventory & impl & derived & $\begin{array}{l}\text { ADM:SG1.S } \\
\text { P3 }\end{array}$ \\
\hline EC-M5 & $\begin{array}{l}\text { percentage of facility assets that have desig- } \\
\text { nated owners but no custodians (if applicable) }\end{array}$ & asset inventory & impl & derived & $\begin{array}{l}\text { ADM:SG1.S } \\
\text { P3 }\end{array}$ \\
\hline EC-M6 & $\begin{array}{l}\text { percentage of facility assets that have desig- } \\
\text { nated custodians but no owners }\end{array}$ & asset inventory & impl & derived & $\begin{array}{l}\text { ADM:SG1.S } \\
\text { P3 }\end{array}$ \\
\hline EC-M7 & $\begin{array}{l}\text { percentage of facility assets that have been } \\
\text { inventoried, by service (if applicable) }\end{array}$ & asset inventory & impl & derived & $\begin{array}{l}\text { ADM:SG2.S } \\
\text { P1 }\end{array}$ \\
\hline EC-M8 & $\begin{array}{l}\text { percentage of facility assets that are not asso- } \\
\text { ciated with one or more services (if applicable) }\end{array}$ & asset inventory & impl & derived & $\begin{array}{l}\text { ADM:SG2.S } \\
\text { P1 }\end{array}$ \\
\hline EC-M9 & $\begin{array}{l}\text { elapsed time since the facility asset inventory } \\
\text { was reviewed }\end{array}$ & asset inventory & impl & $\begin{array}{l}\text { base of } \\
\text { type sche- } \\
\text { dule }\end{array}$ & $\begin{array}{l}\text { ADM:SG1.S } \\
\text { P1 } \\
\text { ADM:SG3.S } \\
\text { P1 }\end{array}$ \\
\hline $\begin{array}{l}\text { EC- } \\
\text { M10 }\end{array}$ & $\begin{array}{l}\text { percentage of facility asset-service dependen- } \\
\text { cy conflicts with unimplemented or incomplete } \\
\text { mitigation plans }\end{array}$ & $\begin{array}{l}\text { asset-service } \\
\text { dependencies }\end{array}$ & impl & derived & $\begin{array}{l}\text { ADM:SG2.S } \\
\text { P2 }\end{array}$ \\
\hline $\begin{array}{l}\text { EC- } \\
\text { M11 }\end{array}$ & $\begin{array}{l}\text { percentage of facility asset-service dependen- } \\
\text { cy conflicts with no mitigation plans }\end{array}$ & $\begin{array}{l}\text { asset-service } \\
\text { dependencies }\end{array}$ & impl & derived & $\begin{array}{l}\text { ADM:SG2.S } \\
\text { P2 }\end{array}$ \\
\hline $\begin{array}{l}\text { EC- } \\
\text { M12 }\end{array}$ & $\begin{array}{l}\text { number of discrepancies between the current } \\
\text { inventory and the previous inventory }\end{array}$ & asset inventory & impl & $\begin{array}{l}\text { base of } \\
\text { type count }\end{array}$ & $\begin{array}{l}\text { ADM:SG3.S } \\
\text { P1 }\end{array}$ \\
\hline $\begin{array}{l}\text { EC- } \\
\text { M13 }\end{array}$ & $\begin{array}{l}\text { number of changes made to asset profiles in } \\
\text { the facility asset inventory }\end{array}$ & asset inventory & impl & $\begin{array}{l}\text { base of } \\
\text { type count }\end{array}$ & $\begin{array}{l}\text { ADM:SG3.S } \\
\text { P2 }\end{array}$ \\
\hline $\begin{array}{l}\text { EC- } \\
\text { M14 }\end{array}$ & $\begin{array}{l}\text { number of changes to resilience requirements } \\
\text { as a result of facility asset changes }\end{array}$ & $\begin{array}{l}\text { asset change } \\
\text { management }\end{array}$ & impl & $\begin{array}{l}\text { base of } \\
\text { type count }\end{array}$ & $\begin{array}{l}\text { ADM:SG3.S } \\
\text { P2 }\end{array}$ \\
\hline $\begin{array}{l}\text { EC- } \\
\text { M15 }\end{array}$ & $\begin{array}{l}\text { number of changes to service continuity plans } \\
\text { as a result of facility asset changes }\end{array}$ & $\begin{array}{l}\text { asset change } \\
\text { management }\end{array}$ & impl & $\begin{array}{l}\text { base of } \\
\text { type count }\end{array}$ & $\begin{array}{l}\text { ADM:SG3.S } \\
\text { P2 }\end{array}$ \\
\hline $\begin{array}{l}\text { EC- } \\
\text { M16 }\end{array}$ & $\begin{array}{l}\text { percentage of facility assets that are desig- } \\
\text { nated as high-value assets }\end{array}$ & asset inventory & impl & derived & EC:SG1.SP1 \\
\hline $\begin{array}{l}\text { EC- } \\
\text { M17 }\end{array}$ & $\begin{array}{l}\text { elapsed time since review and validation of } \\
\text { high-value facility assets and their priorities }\end{array}$ & asset inventory & impl & derived & EC:SG1.SP1 \\
\hline $\begin{array}{l}\text { EC- } \\
\text { M18 }\end{array}$ & $\begin{array}{l}\text { percentage of facility assets that are resi- } \\
\text { lience-focused (those required for service } \\
\text { continuity \& service restoration) }\end{array}$ & asset inventory & impl & derived & EC:SG1.SP2 \\
\hline $\begin{array}{l}\text { EC- } \\
\text { M19 }\end{array}$ & $\begin{array}{l}\text { elapsed time since review and reconciliation } \\
\text { of resilience-focused facility assets }\end{array}$ & asset inventory & impl & derived & EC:SG1.SP2 \\
\hline $\begin{array}{l}\text { EC- } \\
\text { M20 }\end{array}$ & $\begin{array}{l}\text { percentage of facility assets without as- } \\
\text { signed/defined resilience requirements }\end{array}$ & $\begin{array}{l}\text { asset require- } \\
\text { ments }\end{array}$ & impl & derived & EC:SG2.SP1 \\
\hline
\end{tabular}




\begin{tabular}{|c|c|c|c|c|c|}
\hline ID & Measure & $\begin{array}{l}\text { Type of } \\
\text { Information }\end{array}$ & $\begin{array}{l}\text { Measure } \\
\text { Type }\end{array}$ & $\begin{array}{l}\text { Base or } \\
\text { Derived }\end{array}$ & $\begin{array}{l}\text { Applicable } \\
\text { SG.SP }\end{array}$ \\
\hline $\begin{array}{l}\text { EC- } \\
\text { M21 }\end{array}$ & $\begin{array}{l}\text { percentage of facility assets with as- } \\
\text { signed/defined resilience requirements that } \\
\text { are undocumented }\end{array}$ & $\begin{array}{l}\text { asset require- } \\
\text { ments }\end{array}$ & impl & derived & EC:SG2.SP1 \\
\hline $\begin{array}{l}\text { EC- } \\
\text { M22 }\end{array}$ & $\begin{array}{l}\text { percentage of facility assets that do not satisfy } \\
\text { their resilience requirements }\end{array}$ & $\begin{array}{l}\text { asset require- } \\
\text { ment }\end{array}$ & impl & derived & EC:SG2.SP1 \\
\hline $\begin{array}{l}\text { EC- } \\
\text { M23 }\end{array}$ & $\begin{array}{l}\text { percentage of facility assets with no or miss- } \\
\text { ing protection controls }\end{array}$ & asset controls & $\begin{array}{l}\text { impl; } \\
\text { possibly } \\
\text { effec- } \\
\text { tiveness }\end{array}$ & derived & EC:SG2.SP2 \\
\hline $\begin{array}{l}\text { EC- } \\
\text { M24 }\end{array}$ & $\begin{array}{l}\text { percentage of facility assets with no or miss- } \\
\text { ing sustainment controls (including controls } \\
\text { over design, construction, and leasing) }\end{array}$ & asset controls & $\begin{array}{l}\text { impl; } \\
\text { possibly } \\
\text { effec- } \\
\text { tiveness }\end{array}$ & derived & EC:SG2.SP2 \\
\hline $\begin{array}{l}\text { EC- } \\
\text { M25 }\end{array}$ & $\begin{array}{l}\text { percentage of facility asset controls (protec- } \\
\text { tion and sustainment) that are ineffective or } \\
\text { inadequate as demonstrated by: } \\
\text { unsatisfied control objectives } \\
\text { unmet resilience requirements } \\
\text { outstanding control assessment problem } \\
\text { areas above established thresholds and with- } \\
\text { out remediation plans }\end{array}$ & asset controls & $\begin{array}{l}\text { impl; } \\
\text { possibly } \\
\text { effec- } \\
\text { tiveness }\end{array}$ & derived & EC:SG2.SP2 \\
\hline $\begin{array}{l}\text { EC- } \\
\text { M26 }\end{array}$ & $\begin{array}{l}\text { percentage of facility asset control deficien- } \\
\text { cies not resolved by scheduled due date (refer } \\
\text { to CTRL measures for categories of control } \\
\text { deficiencies) }\end{array}$ & asset controls & impl & derived & EC:SG2.SP2 \\
\hline $\begin{array}{l}\text { EC- } \\
\text { M27 }\end{array}$ & $\begin{array}{l}\text { elapsed time since review of the effectiveness } \\
\text { of facility asset controls }\end{array}$ & asset controls & impl & $\begin{array}{l}\text { base of } \\
\text { type sche- } \\
\text { dule }\end{array}$ & EC:SG2.SP2 \\
\hline $\begin{array}{l}\text { EC- } \\
\text { M28 }\end{array}$ & $\begin{array}{l}\text { elapsed time since risk assessment of facility } \\
\text { assets performed }\end{array}$ & asset risk & impl & $\begin{array}{l}\text { base of } \\
\text { type sche- } \\
\text { dule }\end{array}$ & EC:SG3.SP1 \\
\hline $\begin{array}{l}\text { EC- } \\
\text { M29 }\end{array}$ & $\begin{array}{l}\text { percentage of facility assets for which busi- } \\
\text { ness impact valuation }{ }^{24} \text { has not been per- } \\
\text { formed }\end{array}$ & asset risk & impl & derived & EC:SG3.SP1 \\
\hline $\begin{array}{l}\text { EC- } \\
\text { M30 }\end{array}$ & $\begin{array}{l}\text { percentage of facility assets for which a risk } \\
\text { assessment has not been performed and } \\
\text { documented (per policy or other guidelines) } \\
\text { and according to plan }\end{array}$ & asset risk & impl & derived & EC:SG3.SP1 \\
\hline $\begin{array}{l}\text { EC- } \\
\text { M31 }\end{array}$ & $\begin{array}{l}\text { percentage of facility asset risks that have not } \\
\text { been assigned to a responsible party for ac- } \\
\text { tion, tracking, and closure }\end{array}$ & asset risk & impl & derived & EC:SG3.SP2 \\
\hline $\begin{array}{l}\text { EC- } \\
\text { M32 }\end{array}$ & $\begin{array}{l}\text { percentage of facility asset risks }{ }^{25} \text { with a dis- } \\
\text { position of "mitigate or control" that do not } \\
\text { have a defined mitigation plan }\end{array}$ & asset risk & impl & derived & $\underset{26}{E C: S G 3 . S P 2 ~}$ \\
\hline $\begin{array}{l}\text { EC- } \\
\text { M33 }\end{array}$ & $\begin{array}{l}\text { percentage of facility asset risks with a "miti- } \\
\text { gate or control" disposition that are not effec- } \\
\text { tively mitigated by their mitigation plans }\end{array}$ & asset risk & $\begin{array}{l}\text { effec- } \\
\text { tiveness }\end{array}$ & derived & EC:SG3.SP2 \\
\hline
\end{tabular}

24 Business impact valuation can be either qualitative (high, medium, low) or quantitative (based on levels of loss or damage, fines, number of customers lost, disruption in access, etc.).

25 This measure also appears in RISK M4-1. For ease of use of an individual PA (vs. ease of maintenance and consistency), we have decided to replicate some (but not all) risk-related measures in the individual asset PAs that are identified generally in the list of RISK PA measures.

26 SG3.SP2 subpractice 7 states, "Collect performance measures on the risk management process." No such measures are included here in EC; refer to the RISK PA. 


\begin{tabular}{|c|c|c|c|c|c|}
\hline ID & Measure & $\begin{array}{l}\text { Type of } \\
\text { Information }\end{array}$ & $\begin{array}{l}\text { Measure } \\
\text { Type }\end{array}$ & $\begin{array}{l}\text { Base or } \\
\text { Derived }\end{array}$ & $\begin{array}{l}\text { Applicable } \\
\text { SG.SP }\end{array}$ \\
\hline $\begin{array}{l}\text { EC- } \\
\text { M34 }\end{array}$ & $\begin{array}{l}\text { percentage of realized risks for facility assets } \\
\text { that exceed established risk parameters }\end{array}$ & asset risk & $\begin{array}{l}\text { effec- } \\
\text { tiveness }\end{array}$ & derived & EC:SG3.SP2 \\
\hline $\begin{array}{l}\text { EC- } \\
\text { M35 }\end{array}$ & $\begin{array}{l}\text { percentage of facility assets for which a busi- } \\
\text { ness impact analysis has been performed }\end{array}$ & asset continuity & impl & derived & EC:SG4.SP1 \\
\hline $\begin{array}{l}\text { EC- } \\
\text { M36 }\end{array}$ & $\begin{array}{l}\text { elapsed time since business impact analysis } \\
\text { of facility assets performed }\end{array}$ & asset risk & impl & $\begin{array}{l}\text { base of } \\
\text { type sche- } \\
\text { dule }\end{array}$ & $\begin{array}{l}\text { EC:SG3.SP1 } \\
\text { EC:SG4.SP1 }\end{array}$ \\
\hline $\begin{array}{l}\text { EC- } \\
\text { M37 }\end{array}$ & $\begin{array}{l}\text { percentage of facilities with service continuity } \\
\text { plans }\end{array}$ & asset continuity & impl & derived & EC:SG4.SP1 \\
\hline $\begin{array}{l}\text { EC- } \\
\text { M38 }\end{array}$ & $\begin{array}{l}\text { percentage of facilities that are included as } \\
\text { associated assets by service-based continuity } \\
\text { plans }\end{array}$ & asset continuity & impl & derived & EC:SG4.SP1 \\
\hline $\begin{array}{l}\text { EC- } \\
\text { M39 }\end{array}$ & $\begin{array}{l}\text { percentage of external entities that are not } \\
\text { meeting service level agreements for main- } \\
\text { taining facility assets }\end{array}$ & $\begin{array}{l}\text { asset mainten- } \\
\text { ance }\end{array}$ & impl & derived & EC:SG4.SP2 \\
\hline $\begin{array}{l}\text { EC- } \\
\text { M40 }\end{array}$ & $\begin{array}{l}\text { percentage of facility assets that are not main- } \\
\text { tained at required maintenance levels (service } \\
\text { intervals, specifications, etc.) }\end{array}$ & $\begin{array}{l}\text { asset mainten- } \\
\text { ance }\end{array}$ & impl & derived & EC:SG4.SP2 \\
\hline $\begin{array}{l}\text { EC- } \\
\text { M41 }\end{array}$ & $\begin{array}{l}\text { percentage of facility maintenance activities } \\
\text { that are not completed as scheduled }\end{array}$ & $\begin{array}{l}\text { asset mainten- } \\
\text { ance }\end{array}$ & impl & derived & EC:SG4.SP2 \\
\hline $\begin{array}{l}\text { EC- } \\
\text { M42 }\end{array}$ & $\begin{array}{l}\text { elapsed time since facility maintenance per- } \\
\text { formed }\end{array}$ & $\begin{array}{l}\text { asset mainten- } \\
\text { ance }\end{array}$ & impl & $\begin{array}{l}\text { base of } \\
\text { type sche- } \\
\text { dule }\end{array}$ & EC:SG4.SP2 \\
\hline $\begin{array}{l}\text { EC- } \\
\text { M43 }\end{array}$ & $\begin{array}{l}\text { downtime statistics for process control sys- } \\
\text { tems, for example: } \\
\text { physical access systems such as card readers } \\
\text { physical access monitoring such as surveil- } \\
\text { lance cameras } \\
\text { support systems such as HVAC and fire sup- } \\
\text { pression }\end{array}$ & $\begin{array}{l}\text { asset mainten- } \\
\text { ance }\end{array}$ & impl & derived & EC:SG4.SP2 \\
\hline $\begin{array}{l}\text { EC- } \\
\text { M44 }\end{array}$ & $\begin{array}{l}\text { percentage of facilities with dependencies on } \\
\text { public services that are documented in service } \\
\text { continuity plans or other appropriate form }\end{array}$ & $\begin{array}{l}\text { asset depen- } \\
\text { dencies }\end{array}$ & impl & derived & EC:SG4.SP3 \\
\hline $\begin{array}{l}\text { EC- } \\
\text { M45 }\end{array}$ & $\begin{array}{l}\text { percentage of facilities with dependencies on } \\
\text { public infrastructure that are documented in } \\
\text { service continuity plans or other appropriate } \\
\text { form }\end{array}$ & $\begin{array}{l}\text { asset depen- } \\
\text { dencies }\end{array}$ & impl & derived & EC:SG4.SP4 \\
\hline $\begin{array}{l}\text { EC- } \\
\text { M46 }\end{array}$ & $\begin{array}{l}\text { percentage of facilities to be retired with a } \\
\text { plan for facility retirement or, alternatively, a } \\
\text { service continuity plan that addresses facility } \\
\text { retirement }\end{array}$ & $\begin{array}{l}\text { asset retire- } \\
\text { ment }\end{array}$ & impl & derived & EC:SG4.SP5 \\
\hline $\begin{array}{l}\text { EC- } \\
\text { M47 }\end{array}$ & $\begin{array}{l}\text { percentage of facilities planned for retirement } \\
\text { that are not retired according to plan }\end{array}$ & $\begin{array}{l}\text { asset retire- } \\
\text { ment }\end{array}$ & impl & derived & EC:SG4.SP5 \\
\hline $\begin{array}{l}\text { EC- } \\
\text { M48 }\end{array}$ & $\begin{array}{l}\text { number of violations of access control policies } \\
\text { for facility assets }\end{array}$ & policy & impl & $\begin{array}{l}\text { base of } \\
\text { type count }\end{array}$ & $\begin{array}{l}\text { EC:GG2.GP } \\
1\end{array}$ \\
\hline $\begin{array}{l}\text { EC- } \\
\text { M49 }\end{array}$ & $\begin{array}{l}\text { percentage of intrusions into facility assets } \\
\text { where impact exceeds threshold }\end{array}$ & asset intrusions & impl & derived & $\begin{array}{l}\text { none (IMC- } \\
\text { related) }\end{array}$ \\
\hline $\begin{array}{l}\text { EC- } \\
\text { M50 }\end{array}$ & $\begin{array}{l}\text { percentage of clean desk and screen policies } \\
\text { that are met (no violations, all exceptions } \\
\text { approved) }\end{array}$ & policy & impl & derived & $\begin{array}{l}\text { EC:GG2.GP } \\
1\end{array}$ \\
\hline
\end{tabular}




\section{Enterprise Focus (EF)}

The purpose of Enterprise Focus is to establish sponsorship, strategic planning, and governance over the operational resilience management system.

\section{Summary of Specific Goals and Practices}

EF:SG1 Establish Strategic Objectives

EF:SG1.SP1 Establish Strategic Objectives

EF:SG1.SP2 Establish Critical Success Factors

EF:SG1.SP3 Establish Organizational Services

EF:SG2 Plan for Operational Resilience

EF:SG2.SP1 Establish an Operational Resilience Management Plan

EF:SG2.SP2 Establish an Operational Resilience Management Program

\section{EF:SG3 Establish Sponsorship}

EF:SG3.SP1 Commit Funding for Operational Resilience Management

EF:SG3.SP2 Promote a Resilience-Aware Culture

EF:SG3.SP3 Sponsor Resilience Standards and Policies

EF:SG4 Provide Resilience Oversight

EF:SG4.SP1 Establish Resilience as a Governance Focus Area

EF:SG4.SP2 Perform Resilience Oversight

EF:SG4.SP3 Establish Corrective Actions

\section{Measures}

\begin{tabular}{|c|c|c|c|c|c|}
\hline ID & Measure & $\begin{array}{l}\text { Type of } \\
\text { Information }\end{array}$ & $\begin{array}{l}\text { Measure } \\
\text { Type }\end{array}$ & $\begin{array}{l}\text { Base or } \\
\text { Derived }\end{array}$ & $\begin{array}{l}\text { Applicable } \\
\text { SG.SP }\end{array}$ \\
\hline EF-M1 & $\begin{array}{l}\text { percentage of critical success factors that are } \\
\text { attainable per their key performance indicators }\end{array}$ & CSF status & impl & derived & EF:SG1.SP2 \\
\hline EF-M2 & $\begin{array}{l}\text { percentage of services for which a complete } \\
\text { service profile has been documented in the } \\
\text { service repository }\end{array}$ & services & impl & derived & EF:SG1.SP3 \\
\hline EF-M3 & $\begin{array}{l}\text { percentage of services determined to be high- } \\
\text { value }\end{array}$ & services & impl & derived & EF:SG1.SP3 \\
\hline EF-M4 & $\begin{array}{l}\text { percentage of service profiles and service } \\
\text { levels that have been reviewed within their } \\
\text { review time frame }\end{array}$ & services & impl & derived & EF:SG1.SP3 \\
\hline EF-M5 & $\begin{array}{l}\text { percentage of resilience objectives that are } \\
\text { being achieved according to plan }\end{array}$ & plan status & impl & derived & EF:SG2.SP1 \\
\hline EF-M6 & $\begin{array}{l}\text { percentage of operational resilience manage- } \\
\text { ment plan commitments that are being met } \\
\text { according to plan }\end{array}$ & plan status & impl & derived & EF:SG2.SP1 \\
\hline EF-M7 & $\begin{array}{l}\text { percentage of operational resilience manage- } \\
\text { ment program and process activities for which } \\
\text { adequate funds have been allocated }\end{array}$ & $\begin{array}{l}\text { resources } \\
\text { (funding) }\end{array}$ & impl & derived & $\begin{array}{l}\text { EF:SG2.SP2 } \\
\text { EF:SG3.SP1 }\end{array}$ \\
\hline EF-M8 & $\begin{array}{l}\text { percentage of operational resilience manage- } \\
\text { ment program and process activities for which } \\
\text { adequate staff have been allocated }\end{array}$ & $\begin{array}{l}\text { resources } \\
\text { (staff) }\end{array}$ & impl & derived & EF:SG2.SP2 \\
\hline
\end{tabular}




\begin{tabular}{|c|c|c|c|c|c|}
\hline ID & Measure & $\begin{array}{l}\text { Type of } \\
\text { Information }\end{array}$ & $\begin{array}{l}\text { Measure } \\
\text { Type }\end{array}$ & $\begin{array}{l}\text { Base or } \\
\text { Derived }\end{array}$ & $\begin{array}{l}\text { Applicable } \\
\text { SG.SP }\end{array}$ \\
\hline EF-M9 & $\begin{array}{l}\text { percentage of staff demonstrating resilience } \\
\text { awareness commensurate with job descrip- } \\
\text { tions, as measured by the presence of stated } \\
\text { resilience performance goals and objectives } \\
\text { and regular review of these for satisfaction or } \\
\text { correction }\end{array}$ & $\begin{array}{l}\text { cultural aware- } \\
\text { ness }\end{array}$ & impl & derived & EF:SG3.SP2 \\
\hline $\begin{array}{l}\text { EF- } \\
\text { M10 }\end{array}$ & $\begin{array}{l}\text { percentage of external entity relationships for } \\
\text { which resilience requirements have been } \\
\text { specified in the agreements with these entities } \\
\text { (see also EXD) }\end{array}$ & $\begin{array}{l}\text { cultural aware- } \\
\text { ness; candidate } \\
\text { key indicator }\end{array}$ & impl & derived & $\begin{array}{l}E F: S G 3 . S P 2 \\
E F: S G 4 . S P 2\end{array}$ \\
\hline $\begin{array}{l}\text { EF- } \\
\text { M11 }\end{array}$ & $\begin{array}{l}\text { percentage of external entity relationships for } \\
\text { which resilience requirements have been } \\
\text { implemented per the agreements with these } \\
\text { entities (see also EXD) }\end{array}$ & $\begin{array}{l}\text { cultural aware- } \\
\text { ness; candidate } \\
\text { key indicator }\end{array}$ & impl & derived & $\begin{array}{l}E F: S G 3 . S P 2 \\
E F: S G 4 . S P 2\end{array}$ \\
\hline $\begin{array}{l}\text { EF- } \\
\text { M12 }\end{array}$ & $\begin{array}{l}\text { percentage of higher-level managers with } \\
\text { explicit resilience goals }\end{array}$ & sponsorship & impl & derived & EF:SG3.SP2 \\
\hline $\begin{array}{l}\text { EF- } \\
\text { M13 }\end{array}$ & $\begin{array}{l}\text { percentage of higher-level managers who are } \\
\text { promoting and communicating resilience as } \\
\text { measured by satisfactory performance evalua- } \\
\text { tions }\end{array}$ & sponsorship & impl & derived & EF:SG3.SP2 \\
\hline $\begin{array}{l}\text { EF- } \\
\text { M14 }\end{array}$ & $\begin{array}{l}\text { percentage of acculturation of resilience } \\
\text { awareness that is the direct result of sponsor- } \\
\text { ship (by staff group, by organizational unit) }\end{array}$ & sponsorship & impl & derived & EF:SG3.SP2 \\
\hline $\begin{array}{l}\text { EF- } \\
\text { M15 }\end{array}$ & $\begin{array}{l}\text { percentage of higher-level managers that are } \\
\text { fulfilling their commitments to manage resi- } \\
\text { lience per policy as measured by satisfactory } \\
\text { performance evaluations }\end{array}$ & sponsorship & impl & derived & $\begin{array}{l}\text { EF:SG3.SP3 } \\
\text { EF:SG4.SP1 }\end{array}$ \\
\hline $\begin{array}{l}\text { EF- } \\
\text { M16 }\end{array}$ & $\begin{array}{l}\text { percentage of committee charters that include } \\
\text { resilience responsibilities }\end{array}$ & oversight & impl & derived & EF:SG4.SP1 \\
\hline $\begin{array}{l}\text { EF- } \\
\text { M17 }\end{array}$ & $\begin{array}{l}\text { percentage of key operational resilience man- } \\
\text { agement roles for which responsibilities, ac- } \\
\text { countabilities, and authority are assigned and } \\
\text { required skills identified, including key gover- } \\
\text { nance stakeholders }\end{array}$ & oversight & impl & derived & EF:SG4.SP1 \\
\hline $\begin{array}{l}\text { EF- } \\
\text { M18 }\end{array}$ & $\begin{array}{l}\text { percentage of board meetings and/or desig- } \\
\text { nated committee meetings for which opera- } \\
\text { tional resilience management is on the agen- } \\
\text { da }\end{array}$ & oversight & impl & derived & EF:SG4.SP1 \\
\hline $\begin{array}{l}\text { EF- } \\
\text { M19 }\end{array}$ & $\begin{array}{l}\text { percentage of key indicators (KPIs, KRIs, } \\
\mathrm{KCls} \text { ) that are within acceptable ranges }\end{array}$ & oversight & impl & derived & EF:SG4.SP2 \\
\hline $\begin{array}{l}\text { EF- } \\
\text { M20 }\end{array}$ & $\begin{array}{l}\text { percentage of key indicators that are outside } \\
\text { of acceptable ranges and for which a correc- } \\
\text { tive action plan exists }\end{array}$ & oversight & impl & derived & $\begin{array}{l}E F: S G 4 . S P 2 \\
E F: S G 4 . S P 3\end{array}$ \\
\hline $\begin{array}{l}\text { EF- } \\
\text { M21 }\end{array}$ & $\begin{array}{l}\text { percentage of key indicators with corrective } \\
\text { action plans where actions taken were suc- } \\
\text { cessful in bringing indicators within acceptable } \\
\text { ranges }\end{array}$ & oversight & impl & derived & EF:SG4.SP3 \\
\hline $\begin{array}{l}\text { EF- } \\
\text { M22 }\end{array}$ & $\begin{array}{l}\text { elapsed calendar time since key indicators } \\
\text { were reported to governance stakeholders }\end{array}$ & oversight & impl & $\begin{array}{l}\text { base of } \\
\text { type sche- } \\
\text { dule }\end{array}$ & EF:SG4.SP2 \\
\hline $\begin{array}{l}\text { EF- } \\
\text { M23 }\end{array}$ & $\begin{array}{l}\text { percentage of required internal and external } \\
\text { audits completed and reviewed by the board } \\
\text { or other designated oversight body }\end{array}$ & oversight & impl & derived & EF:SG4.SP2 \\
\hline $\begin{array}{l}\text { EF- } \\
\text { M24 }\end{array}$ & $\begin{array}{l}\text { percentage of audit findings that have been } \\
\text { resolved }\end{array}$ & oversight & impl & derived & EF:SG4.SP2 \\
\hline
\end{tabular}




\begin{tabular}{|c|c|c|c|c|c|}
\hline ID & Measure & $\begin{array}{l}\text { Type of } \\
\text { Information }\end{array}$ & $\begin{array}{l}\text { Measure } \\
\text { Type }\end{array}$ & $\begin{array}{l}\text { Base or } \\
\text { Derived }\end{array}$ & $\begin{array}{l}\text { Applicable } \\
\text { SG.SP }\end{array}$ \\
\hline $\begin{array}{l}\text { EF- } \\
\text { M25 }\end{array}$ & $\begin{array}{l}\text { percentage of incidents that caused damage, } \\
\text { compromise, or loss beyond established thre- } \\
\text { sholds to the organization's assets and ser- } \\
\text { vices (categorized by asset, by service, by } \\
\text { incident type, etc.) }\end{array}$ & $\begin{array}{l}\text { candidate key } \\
\text { indicator }\end{array}$ & impl & derived & EF:SG4.SP2 \\
\hline $\begin{array}{l}\text { EF- } \\
\text { M26 }\end{array}$ & $\begin{array}{l}\text { dollar amount of estimated damage or loss } \\
\text { resulting from all incidents (categorized by } \\
\text { asset, by service, by incident type, etc.) }\end{array}$ & $\begin{array}{l}\text { candidate key } \\
\text { indicator }\end{array}$ & impl & $\begin{array}{l}\text { base of } \\
\text { type cost }\end{array}$ & EF:SG4.SP2 \\
\hline $\begin{array}{l}\text { EF- } \\
\text { M27 }\end{array}$ & $\begin{array}{l}\text { percentage of organizational units with estab- } \\
\text { lished service continuity plan(s) for the servic- } \\
\text { es that require such a plan where the unit is } \\
\text { the designated owner }\end{array}$ & $\begin{array}{l}\text { candidate key } \\
\text { indicator }\end{array}$ & impl & derived & $\begin{array}{l}\text { EF:SG4.SP2 } \\
\text { SC:SG3.SP1 }\end{array}$ \\
\hline $\begin{array}{l}\text { EF- } \\
\text { M28 }\end{array}$ & $\begin{array}{l}\text { percentage of key external resilience require- } \\
\text { ments (laws, regulations, standards, etc.) for } \\
\text { which the organization has been deemed by } \\
\text { objective audit to be in compliance (see also } \\
\text { COMP) }\end{array}$ & $\begin{array}{l}\text { candidate key } \\
\text { indicator }\end{array}$ & impl & derived & EF:SG4.SP2 \\
\hline $\begin{array}{l}\text { EF- } \\
\text { M29 }\end{array}$ & $\begin{array}{l}\text { level of capability achieved in other operation- } \\
\text { al resilience management process areas }\end{array}$ & $\begin{array}{l}\text { candidate key } \\
\text { indicator }\end{array}$ & impl & $\begin{array}{l}\text { base of } \\
\text { type or- } \\
\text { dinal/ratio }\end{array}$ & EF:SG4.SP2 \\
\hline $\begin{array}{l}\text { EF- } \\
\text { M30 }\end{array}$ & $\begin{array}{l}\text { percentage of operational resilience manage- } \\
\text { ment policies that are met }\end{array}$ & $\begin{array}{l}\text { candidate key } \\
\text { indicator }\end{array}$ & impl & derived & EF:SG4.SP2 \\
\hline $\begin{array}{l}\text { EF- } \\
\text { M31 }\end{array}$ & $\begin{array}{l}\text { number of policy violations for policies related } \\
\text { to each operational resilience management } \\
\text { process area }\end{array}$ & $\begin{array}{l}\text { candidate key } \\
\text { indicator }\end{array}$ & impl & $\begin{array}{l}\text { base of } \\
\text { type count }\end{array}$ & EF:SG4.SP2 \\
\hline $\begin{array}{l}\text { EF- } \\
\text { M32 }\end{array}$ & $\begin{array}{l}\text { percentage of high-value assets (by asset } \\
\text { type) for which a comprehensive strategy and } \\
\text { internal control system have been imple- } \\
\text { mented to mitigate risks as necessary and to } \\
\text { maintain these risks within acceptable thre- } \\
\text { sholds }\end{array}$ & $\begin{array}{l}\text { candidate key } \\
\text { indicator }\end{array}$ & impl & derived & EF:SG4.SP2 \\
\hline $\begin{array}{l}\text { EF- } \\
\text { M33 }\end{array}$ & $\begin{array}{l}\text { number of enterprise-level risks referred to the } \\
\text { risk management process }\end{array}$ & $\begin{array}{l}\text { risk identifica- } \\
\text { tion }\end{array}$ & impl & $\begin{array}{l}\text { base of } \\
\text { type count }\end{array}$ & EF:SG4.SP2 \\
\hline $\begin{array}{l}\text { EF- } \\
\text { M34 }\end{array}$ & $\begin{array}{l}\text { percentage of CERT-RMM practices (based } \\
\text { on a specific model scope) that are addressed } \\
\text { by governance (EF) activities }\end{array}$ & $\begin{array}{l}\text { governance } \\
\text { scope }\end{array}$ & $\begin{array}{l}\text { impl; } \\
\text { possibly } \\
\text { effec- } \\
\text { tiveness }\end{array}$ & derived & none \\
\hline
\end{tabular}

\section{External Dependencies Management (EXD)}

The purpose of External Dependencies Management is to establish and manage an appropriate level of controls to ensure the resilience of services and assets that are dependent on the actions of external entities.

\section{Summary of Specific Goals and Practices}

EXD:SG1 Identify and Prioritize External Dependencies

EXD:SG1.SP1 Identify External Dependencies

EXD:SG1.SP2 Prioritize External Dependencies

EXD:SG2 Manage Risks Due to External Dependencies

EXD:SG2.SP1 Identify and Assess Risks Due to External Dependencies

EXD:SG2.SP2 Mitigate Risks Due to External Dependencies 
EXD:SG3 Establish Formal Relationships

EXD:SG3.SP1 Establish Enterprise Specifications for External Dependencies

EXD:SG3.SP2 Establish Resilience Specifications for External Dependencies

EXD:SG3.SP3 Evaluate and Select External Entities

EXD:SG3.SP4 Formalize Relationships

EXD:SG4 Manage External Entity Performance

EXD:SG4.SP1 Monitor External Entity Performance

EXD:SG4.SP2 Correct External Entity Performance

\section{Measures}

\begin{tabular}{|c|c|c|c|c|c|}
\hline ID & Measure & $\begin{array}{l}\text { Type of } \\
\text { Information }\end{array}$ & $\begin{array}{l}\text { Measure } \\
\text { Type }\end{array}$ & $\begin{array}{l}\text { Base or } \\
\text { Derived }\end{array}$ & $\begin{array}{l}\text { Applicable } \\
\text { SG.SP }\end{array}$ \\
\hline $\begin{array}{l}\text { EXD- } \\
\text { M1 }\end{array}$ & $\begin{array}{l}\text { by external dependency, in priority order: } \\
\text { percentage of services that rely on the exter- } \\
\text { nal dependency } \\
\text { percentage of assets that rely on the external } \\
\text { dependency }\end{array}$ & $\begin{array}{l}\text { definition of } \\
\text { external } \\
\text { dependen- } \\
\text { cies }\end{array}$ & impl & derived & $\begin{array}{l}\text { EXD:SG1.S } \\
\text { P1 } \\
\text { EXD:SG1.S } \\
\text { P2 }\end{array}$ \\
\hline $\begin{array}{l}\text { EXD- } \\
\text { M2 }\end{array}$ & $\begin{array}{l}\text { by external entity, in priority order: } \\
\text { number of services that rely on }{ }^{27} \text { the external } \\
\text { entity, by type of service (if applicable) } \\
\text { number of assets that rely on the external } \\
\text { entity, by type of asset } \\
\text { number of external dependencies which rely } \\
\text { on the external entity } \\
\text { number of compliance obligations that rely on } \\
\text { or apply to the external entity } \\
\text { monetary value of the relationship with the } \\
\text { external entity } \\
\text { number of agreement changes by change } \\
\text { type } \\
\text { number of entities external to itself upon which } \\
\text { the external entity relies to meet its obligations }\end{array}$ & $\begin{array}{l}\text { identification } \\
\text { of external } \\
\text { entities }\end{array}$ & impl & $\begin{array}{l}\text { base of } \\
\text { type count }\end{array}$ & $\begin{array}{l}\text { EXD:SG1.S } \\
\text { P1 } \\
\text { EXD:SG1.S } \\
\text { P2 }^{29}\end{array}$ \\
\hline $\begin{array}{l}\text { EXD- } \\
\text { M3 }\end{array}$ & $\begin{array}{l}\text { percentage of assets that rely on external } \\
\text { entities }\end{array}$ & $\begin{array}{l}\text { identification } \\
\text { of external } \\
\text { entities }\end{array}$ & impl & derived & $\begin{array}{l}\text { EXD:SG1.S } \\
\text { P1 } \\
\text { EXD:SG1.S } \\
\text { P2 }\end{array}$ \\
\hline $\begin{array}{l}\text { EXD- } \\
\text { M4 }\end{array}$ & $\begin{array}{l}\text { percentage of services that rely on external } \\
\text { entities }\end{array}$ & $\begin{array}{l}\text { identification } \\
\text { of external } \\
\text { entities }\end{array}$ & impl & derived & $\begin{array}{l}\text { EXD:SG1.S } \\
\text { P1 } \\
\text { EXD:SG1.S } \\
\text { P2 }\end{array}$ \\
\hline
\end{tabular}

27 "Rely on" includes accessed, owned, responsible for, developed, controlled, used, operated, or otherwise influenced by the external entity.

28 This should be supported by some type of visual traceability mapping that shows the relationships between external entities and external dependencies.

29 Prioritization of external entities not explicitly addressed in SG1.SP2 but can be inferred 


\begin{tabular}{|c|c|c|c|c|c|}
\hline ID & Measure & $\begin{array}{l}\text { Type of } \\
\text { Information }\end{array}$ & $\begin{array}{l}\text { Measure } \\
\text { Type }\end{array}$ & $\begin{array}{l}\text { Base or } \\
\text { Derived }\end{array}$ & $\begin{array}{l}\text { Applicable } \\
\text { SG.SP }\end{array}$ \\
\hline $\begin{array}{l}\text { EXD- } \\
\text { M5 }\end{array}$ & $\begin{array}{l}\text { number of external entities by relationship } \\
\text { status (RFP, source selection, awarded, } \\
\text { agreement/contract executed, performing as } \\
\text { expected, out of compliance, in dispute or } \\
\text { litigation, terminated, renewed, etc.) }\end{array}$ & $\begin{array}{l}\text { identification } \\
\text { of external } \\
\text { entities }\end{array}$ & impl & $\begin{array}{l}\text { base of } \\
\text { type count }\end{array}$ & $\begin{array}{l}\text { EXD:SG1.S } \\
\text { P1 } \\
\text { EXD:SG3.S } \\
\text { P4 } \\
\text { EXD:SG4.S } \\
\text { P1 } \\
\text { EXD:SG4.S } \\
\text { P2 }\end{array}$ \\
\hline $\begin{array}{l}\text { EXD- } \\
\text { M6 }\end{array}$ & $\begin{array}{l}\text { number of external entities at each CERT- } \\
\text { RMM capability level by process area }\end{array}$ & $\begin{array}{l}\text { definition of } \\
\text { external } \\
\text { entities }\end{array}$ & impl & $\begin{array}{l}\text { base of } \\
\text { type count }\end{array}$ & none \\
\hline $\begin{array}{l}\text { EXD- } \\
\text { M7 }\end{array}$ & $\begin{array}{l}\text { percentage of external dependencies without } \\
\text { a designated owner }\end{array}$ & $\begin{array}{l}\text { definition of } \\
\text { external } \\
\text { dependen- } \\
\text { cies }\end{array}$ & impl & derived & $\begin{array}{l}\text { EXD:SG1.S } \\
\text { P1 }\end{array}$ \\
\hline $\begin{array}{l}\text { EXD- } \\
\text { M8 }\end{array}$ & $\begin{array}{l}\text { percentage of external entities without a des- } \\
\text { ignated owner }\end{array}$ & $\begin{array}{l}\text { identification } \\
\text { of external } \\
\text { entities }\end{array}$ & impl & derived & $\begin{array}{l}\text { EXD:SG1.S } \\
\text { P1 }\end{array}$ \\
\hline $\begin{array}{l}\text { EXD- } \\
\text { M9 }\end{array}$ & $\begin{array}{l}\text { percentage of external dependencies involved } \\
\text { in meeting compliance obligations }\end{array}$ & $\begin{array}{l}\text { definition of } \\
\text { external } \\
\text { dependen- } \\
\text { cies }\end{array}$ & impl & derived & $\begin{array}{l}\text { EXD:SG1.S } \\
\text { P1 }\end{array}$ \\
\hline $\begin{array}{l}\text { EXD- } \\
\text { M10 }\end{array}$ & $\begin{array}{l}\text { percentage of external entities involved in } \\
\text { meeting compliance obligations }\end{array}$ & $\begin{array}{l}\text { identification } \\
\text { of external } \\
\text { entities }\end{array}$ & impl & derived & $\begin{array}{l}\text { EXD:SG1.S } \\
\text { P1 }\end{array}$ \\
\hline $\begin{array}{l}\text { EXD- } \\
\text { M11 }\end{array}$ & $\begin{array}{l}\text { number of external entities that are providing } \\
\text { "commodity" services (easily replaced) }\end{array}$ & $\begin{array}{l}\text { identification } \\
\text { of external } \\
\text { entities }\end{array}$ & impl & $\begin{array}{l}\text { base of } \\
\text { type count }\end{array}$ & $\begin{array}{l}\text { EXD:SG1.S } \\
\text { P1 }\end{array}$ \\
\hline $\begin{array}{l}\text { EXD- } \\
\text { M12 }\end{array}$ & $\begin{array}{l}\text { number of external entities that are providing } \\
\text { "specialized" services (difficult to replace) }\end{array}$ & $\begin{array}{l}\text { identification } \\
\text { of external } \\
\text { entities }\end{array}$ & impl & $\begin{array}{l}\text { base of } \\
\text { type count }\end{array}$ & $\begin{array}{l}\text { EXD:SG1.S } \\
\text { P1 }\end{array}$ \\
\hline $\begin{array}{l}\text { EXD- } \\
\text { M13 }\end{array}$ & $\begin{array}{l}\text { number of external entities in the same geo- } \\
\text { graphic region (for assessing geographic and } \\
\text { socio-political risk) }\end{array}$ & $\begin{array}{l}\text { identification } \\
\text { of external } \\
\text { entities }\end{array}$ & impl & $\begin{array}{l}\text { base of } \\
\text { type count }\end{array}$ & $\begin{array}{l}\text { EXD:SG1.S } \\
\text { P1 }\end{array}$ \\
\hline $\begin{array}{l}\text { EXD- } \\
\text { M14 }\end{array}$ & $\begin{array}{l}\text { number of external entities for which the rela- } \\
\text { tionship is managed by another part of the } \\
\text { organization than the one owning the relation- } \\
\text { ship }\end{array}$ & $\begin{array}{l}\text { identification } \\
\text { of external } \\
\text { entities }\end{array}$ & impl & $\begin{array}{l}\text { base of } \\
\text { type count }\end{array}$ & $\begin{array}{l}\text { EXD:SG1.S } \\
\text { P1 }\end{array}$ \\
\hline $\begin{array}{l}\text { EXD- } \\
\text { M15 }\end{array}$ & $\begin{array}{l}\text { percentage of external dependencies that } \\
\text { have not been reviewed and updated as } \\
\text { scheduled }\end{array}$ & $\begin{array}{l}\text { update of } \\
\text { external } \\
\text { dependen- } \\
\text { cies }\end{array}$ & impl & derived & $\begin{array}{l}\text { EXD:SG1.S } \\
\text { P1 } \\
\text { EXD:SG3.S } \\
\text { P1 } \\
\text { EXD:SG3.S } \\
\text { P2 }\end{array}$ \\
\hline $\begin{array}{l}\text { EXD- } \\
\text { M16 }\end{array}$ & $\begin{array}{l}\text { elapsed time since risk assessment of exter- } \\
\text { nal dependencies }\end{array}$ & $\begin{array}{l}\text { external } \\
\text { dependency } \\
\text { risk }\end{array}$ & impl & $\begin{array}{l}\text { base of } \\
\text { type sche- } \\
\text { dule }\end{array}$ & $\begin{array}{l}\text { EXD:SG2.S } \\
\text { P1 }\end{array}$ \\
\hline $\begin{array}{l}\text { EXD- } \\
\text { M17 }\end{array}$ & $\begin{array}{l}\text { percentage of external dependencies for } \\
\text { which a risk assessment has not been per- } \\
\text { formed and documented (per policy or other } \\
\text { guidelines) according to plan }\end{array}$ & $\begin{array}{l}\text { external } \\
\text { dependency } \\
\text { risk }\end{array}$ & impl & derived & $\begin{array}{l}\text { EXD:SG2.S } \\
\text { P1 }\end{array}$ \\
\hline $\begin{array}{l}\text { EXD- } \\
\text { M18 }\end{array}$ & $\begin{array}{l}\text { percentage of external dependency risks that } \\
\text { have not been assigned to a responsible party } \\
\text { for action, tracking, and closure }\end{array}$ & $\begin{array}{l}\text { external } \\
\text { dependency } \\
\text { risk }\end{array}$ & impl & derived & $\begin{array}{l}\text { EXD:SG2.S } \\
\text { P2 }\end{array}$ \\
\hline
\end{tabular}

30 A CERT-RMM class A appraisal is required to assign a capability level. All external entities may not have performed such an appraisal. 


\begin{tabular}{|c|c|c|c|c|c|}
\hline ID & Measure & $\begin{array}{l}\text { Type of } \\
\text { Information }\end{array}$ & $\begin{array}{l}\text { Measure } \\
\text { Type }\end{array}$ & $\begin{array}{l}\text { Base or } \\
\text { Derived }\end{array}$ & $\begin{array}{l}\text { Applicable } \\
\text { SG.SP }\end{array}$ \\
\hline $\begin{array}{l}\text { EXD- } \\
\text { M19 }\end{array}$ & $\begin{array}{l}\text { percentage of external dependency risks }{ }^{31} \\
\text { with a disposition of "mitigate or control" that } \\
\text { do not have a defined mitigation plan }\end{array}$ & $\begin{array}{l}\text { external } \\
\text { dependency } \\
\text { risk }\end{array}$ & impl & derived & $\begin{array}{l}\text { EXD:SG2.S } \\
\text { P2 }\end{array}$ \\
\hline $\begin{array}{l}\text { EXD- } \\
\text { M20 }\end{array}$ & $\begin{array}{l}\text { percentage of external dependency risks with } \\
\text { a "mitigate or control" disposition that are not } \\
\text { effectively mitigated by their mitigation plans }\end{array}$ & $\begin{array}{l}\text { external } \\
\text { dependency } \\
\text { risk }\end{array}$ & impl & derived & $\begin{array}{l}\text { EXD:SG2.S } \\
\text { P2 }\end{array}$ \\
\hline $\begin{array}{l}\text { EXD- } \\
\text { M21 }\end{array}$ & $\begin{array}{l}\text { percentage of realized risks for external de- } \\
\text { pendencies that exceed established risk pa- } \\
\text { rameters }\end{array}$ & $\begin{array}{l}\text { external } \\
\text { dependency } \\
\text { risk }\end{array}$ & effectiveness & derived & $\begin{array}{l}\text { EXD:SG2.S } \\
\text { P2 }\end{array}$ \\
\hline $\begin{array}{l}\text { EXD- } \\
\text { M22 }\end{array}$ & $\begin{array}{l}\text { percentage of RFPs for external entities that } \\
\text { do not include resilience specifications }\end{array}$ & $\begin{array}{l}\text { external } \\
\text { entity selec- } \\
\text { tion }\end{array}$ & impl & derived & $\begin{array}{l}\text { EXD:SG3.S } \\
\text { P3 }\end{array}$ \\
\hline $\begin{array}{l}\text { EXD- } \\
\text { M23 }\end{array}$ & $\begin{array}{l}\text { percentage of candidate external entities } \\
\text { whose due diligence process is on track per } \\
\text { plan }\end{array}$ & $\begin{array}{l}\text { external } \\
\text { entity selec- } \\
\text { tion }\end{array}$ & impl & derived & $\begin{array}{l}\text { EXD:SG3.S } \\
\text { P3 }\end{array}$ \\
\hline $\begin{array}{l}\text { EXD- } \\
\text { M24 }\end{array}$ & $\begin{array}{l}\text { percentage of selected external entities with- } \\
\text { out documented selection and decision ratio- } \\
\text { nale (this should be zero) }\end{array}$ & $\begin{array}{l}\text { external } \\
\text { entity selec- } \\
\text { tion }\end{array}$ & impl & derived & $\begin{array}{l}\text { EXD:SG3.S } \\
\text { P3 }\end{array}$ \\
\hline $\begin{array}{l}\text { EXD- } \\
\text { M25 }\end{array}$ & $\begin{array}{l}\text { number of resilience specifications unmet by } \\
\text { the selected external entity }\end{array}$ & $\begin{array}{l}\text { external } \\
\text { entity selec- } \\
\text { tion }\end{array}$ & impl & $\begin{array}{l}\text { base of } \\
\text { type count }\end{array}$ & $\begin{array}{l}\text { EXD:SG3.S } \\
\text { P3 }\end{array}$ \\
\hline $\begin{array}{l}\text { EXD- } \\
\text { M26 }\end{array}$ & $\begin{array}{l}\text { number of resilience specifications unmet by } \\
\text { the selected external entity that are identified } \\
\text { as risks to be managed (ranked) }\end{array}$ & $\begin{array}{l}\text { external } \\
\text { entity selec- } \\
\text { tion }\end{array}$ & impl & $\begin{array}{l}\text { base of } \\
\text { type count }\end{array}$ & $\begin{array}{l}\text { EXD:SG3.S } \\
\text { P3 }\end{array}$ \\
\hline $\begin{array}{l}\text { EXD- } \\
\text { M27 }\end{array}$ & $\begin{array}{l}\text { percentage of agreements/contracts with } \\
\text { external entities with specifications that have } \\
\text { been waived as a result of negotiations }\end{array}$ & $\begin{array}{l}\text { external } \\
\text { entity } \\
\text { agreements }\end{array}$ & impl & derived & $\begin{array}{l}\text { EXD:SG3.S } \\
\text { P4 }\end{array}$ \\
\hline $\begin{array}{l}\text { EXD- } \\
\text { M28 }\end{array}$ & $\begin{array}{l}\text { percentage of external entities that are achiev- } \\
\text { ing all specifications as defined in the agree- } \\
\text { ment }\end{array}$ & $\begin{array}{l}\text { external } \\
\text { entity } \\
\text { agreements }\end{array}$ & impl & derived & $\begin{array}{l}\text { EXD:SG4.S } \\
\text { P1 }\end{array}$ \\
\hline $\begin{array}{l}\text { EXD- } \\
\text { M29 }\end{array}$ & $\begin{array}{l}\text { percentage of external entity agreements that } \\
\text { have not been reviewed as scheduled (includ- } \\
\text { ing in response to changes in enterprise and } \\
\text { resilience specifications) }\end{array}$ & $\begin{array}{l}\text { external } \\
\text { entity status }\end{array}$ & impl & derived & $\begin{array}{l}\text { EXD:SG3.S } \\
\text { P1 } \\
\text { EXD:SG2.S } \\
\text { P2 }\end{array}$ \\
\hline $\begin{array}{l}\text { EXD- } \\
\text { M30 }\end{array}$ & $\begin{array}{l}\text { percentage of external entities whose status } \\
\text { (monitoring and inspection activities) has not } \\
\text { been reviewed as scheduled }\end{array}$ & $\begin{array}{l}\text { external } \\
\text { entity status }\end{array}$ & impl & derived & $\begin{array}{l}\text { EXD:SG4.S } \\
\text { P1 }\end{array}$ \\
\hline $\begin{array}{l}\text { EXD- } \\
\text { M31 }\end{array}$ & $\begin{array}{l}\text { percentage of external entities that have un- } \\
\text { dergone, as required by agreement/contract: } \\
\text { - reviews } \\
\text { - risk assessments } \\
\text { - testing, evaluations } \\
\text { - inspections } \\
\text { - audits }\end{array}$ & $\begin{array}{l}\text { external } \\
\text { entity status }\end{array}$ & impl & derived & $\begin{array}{l}\text { EXD:SG4.S } \\
\text { P1 }\end{array}$ \\
\hline $\begin{array}{l}\text { EXD- } \\
\text { M32 }\end{array}$ & $\begin{array}{l}\text { percentage of external entities with corrective } \\
\text { actions that have not been implemented as } \\
\text { scheduled }\end{array}$ & $\begin{array}{l}\text { external } \\
\text { entity status }\end{array}$ & impl & derived & $\begin{array}{l}\text { EXD:SG4.S } \\
\text { P2 }\end{array}$ \\
\hline $\begin{array}{l}\text { EXD- } \\
\text { M33 }\end{array}$ & $\begin{array}{l}\text { percentage of external entities whose delive- } \\
\text { rables have failed to pass inspection }\end{array}$ & $\begin{array}{l}\text { external } \\
\text { entity status }\end{array}$ & impl & derived & $\begin{array}{l}\text { EXD:SG4.S } \\
\text { P1 }\end{array}$ \\
\hline
\end{tabular}

31 This measure also appears in RISK M4-1. For ease of use of an individual PA (vs. ease of maintenance and consistency), we have decided to replicate some (but not all) risk-related measures in the individual asset PAs that are identified generally in the list of RISK PA measures. 


\begin{tabular}{|c|c|c|c|c|c|}
\hline ID & Measure & $\begin{array}{l}\text { Type of } \\
\text { Information }\end{array}$ & $\begin{array}{l}\text { Measure } \\
\text { Type }\end{array}$ & $\begin{array}{l}\text { Base or } \\
\text { Derived }\end{array}$ & $\begin{array}{l}\text { Applicable } \\
\text { SG.SP }\end{array}$ \\
\hline $\begin{array}{l}\text { EXD- } \\
\text { M34 }\end{array}$ & $\begin{array}{l}\text { for all or specific external entities, elapsed } \\
\text { time since last: } \\
\text { - risk assessment } \\
\text { - performance review } \\
\text { - compliance audit } \\
\text { - joint service continuity exercise }\end{array}$ & $\begin{array}{l}\text { external } \\
\text { entity status; } \\
\text { external } \\
\text { entity risk }\end{array}$ & impl & $\begin{array}{l}\text { base of } \\
\text { type sche- } \\
\text { dule }\end{array}$ & $\begin{array}{l}\text { EXD:SG4.S } \\
\text { P1 }\end{array}$ \\
\hline $\begin{array}{l}\text { EXD- } \\
\text { M35 }\end{array}$ & $\begin{array}{l}\text { for all applicable external entities, elapsed } \\
\text { time since source code was last updated in } \\
\text { source code escrow }\end{array}$ & $\begin{array}{l}\text { external } \\
\text { entity status }\end{array}$ & impl & $\begin{array}{l}\text { base of } \\
\text { type sche- } \\
\text { dule }\end{array}$ & $\begin{array}{l}\text { EXD:SG4.S } \\
\text { P1 }\end{array}$ \\
\hline $\begin{array}{l}\text { EXD- } \\
\text { M36 }\end{array}$ & $\begin{array}{l}\text { percentage of external entity risks that have } \\
\text { not been assigned to a responsible party for } \\
\text { action, tracking, and closure }\end{array}$ & $\begin{array}{l}\text { external } \\
\text { entity risk }\end{array}$ & impl & derived & $\begin{array}{l}\text { EXD:SG4.S } \\
\text { P1 }\end{array}$ \\
\hline $\begin{array}{l}\text { EXD- } \\
\text { M37 }\end{array}$ & $\begin{array}{l}\text { percentage of realized risks for external enti- } \\
\text { ties that exceed established risk parameters }\end{array}$ & $\begin{array}{l}\text { external } \\
\text { entity risk }\end{array}$ & effectiveness & derived & $\begin{array}{l}\text { EXD:SG4.S } \\
\text { P1 }\end{array}$ \\
\hline $\begin{array}{l}\text { EXD- } \\
\text { M38 }\end{array}$ & $\begin{array}{l}\text { percentage of external entities whose financial } \\
\text { health is at risk (beyond risk parameters) }\end{array}$ & $\begin{array}{l}\text { external } \\
\text { entity risk }\end{array}$ & impl & derived & $\begin{array}{l}\text { EXD:SG4ss } \\
\text { sss.SP1 }\end{array}$ \\
\hline $\begin{array}{l}\text { EXD- } \\
\text { M39 }\end{array}$ & $\begin{array}{l}\text { percentage of external entities whose perfor- } \\
\text { mance deviates sufficiently from specifications } \\
\text { (beyond risk parameters) to cause a risk to be } \\
\text { referred to the risk management process }\end{array}$ & $\begin{array}{l}\text { external } \\
\text { entity risk }\end{array}$ & impl & derived & $\begin{array}{l}\text { EXD:SG4.S } \\
\text { P1 }\end{array}$ \\
\hline $\begin{array}{l}\text { EXD- } \\
\text { M40 }\end{array}$ & $\begin{array}{l}\text { percentage of external entities that play a key } \\
\text { role in fulfilling service continuity plans during } \\
\text { disruptive events }\end{array}$ & $\begin{array}{l}\text { external } \\
\text { entity service } \\
\text { continuity }\end{array}$ & impl & derived & none \\
\hline $\begin{array}{l}\text { EXD- } \\
\text { M41 }\end{array}$ & $\begin{array}{l}\text { percentage of external entities that have } \\
\text { tested their service continuity plans, including } \\
\text { participating in tests conducted of organiza- } \\
\text { tion's service continuity plans }\end{array}$ & $\begin{array}{l}\text { external } \\
\text { entity service } \\
\text { continuity }\end{array}$ & impl & derived & none \\
\hline $\begin{array}{l}\text { EXD- } \\
\text { M42 }\end{array}$ & $\begin{array}{l}\text { percentage of external entities that failed to } \\
\text { perform as expected during a disruptive event }\end{array}$ & $\begin{array}{l}\text { external } \\
\text { entity service } \\
\text { continuity }\end{array}$ & impl & derived & none \\
\hline
\end{tabular}

\section{Financial Resource Management (FRM)}

The purpose of Financial Resource Management is to request, receive, manage, and apply financial resources to support resilience objectives and requirements.

\section{Summary of Specific Goals and Practices}

FRM:SG1 Establish Financial Commitment

FRM:SG1.SP1 Commit Funding for Operational Resilience Management

FRM:SG1.SP2 Establish Structure to Support Financial Management

FRM:SG2 Perform Financial Planning

FRM:SG2.SP1 Define Funding Needs

FRM:SG2.SP2 Establish Resilience Budgets

FRM:SG2.SP3 Resolve Funding Gaps

FRM:SG3 Fund Resilience Activities

FRM:SG3.SP1 Fund Resilience Activities

FRM:SG4 Account for Resilience Activities

FRM:SG4.SP1 Track and Document Costs

FRM:SG4.SP2 Perform Cost and Performance Analysis 
FRM:SG5 Optimize Resilience Expenditures and Investments

FRM:SG5.SP1 Optimize Resilience Expenditures

FRM:SG5.SP2 Determine Return on Resilience Investments

FRM:SG5.SP3 Identify Cost Recovery Opportunities

\section{Measures}

\begin{tabular}{|c|c|c|c|c|c|}
\hline ID & Measure & $\begin{array}{l}\text { Type of } \\
\text { Information }\end{array}$ & $\begin{array}{l}\text { Measure } \\
\text { Type }\end{array}$ & $\begin{array}{l}\text { Base or } \\
\text { Derived }\end{array}$ & $\begin{array}{l}\text { Applicable } \\
\text { SG.SP }\end{array}$ \\
\hline $\begin{array}{l}\text { FRM- } \\
\text { M1 }\end{array}$ & $\begin{array}{l}\text { elapsed time since the business case for } \\
\text { the operational resilience management } \\
\text { (ORM) system was reviewed and updated }\end{array}$ & $\begin{array}{l}\text { resilience } \\
\text { business } \\
\text { case }\end{array}$ & impl & $\begin{array}{l}\text { base of type } \\
\text { schedule }\end{array}$ & FRM:SG1.SP1 \\
\hline $\begin{array}{l}\text { FRM- } \\
\text { M2 }\end{array}$ & $\begin{array}{l}\text { elapsed time since ORM system funding } \\
\text { was reviewed }\end{array}$ & $\begin{array}{l}\text { resilience } \\
\text { funding }\end{array}$ & impl & $\begin{array}{l}\text { base of type } \\
\text { schedule }\end{array}$ & FRM:SG1.SP1 \\
\hline $\begin{array}{l}\text { FRM- } \\
\text { M3 }\end{array}$ & $\begin{array}{l}\text { elapsed time since ORM system funding } \\
\text { was reviewed as part of the organization's } \\
\text { strategic plan budgeting exercise }\end{array}$ & $\begin{array}{l}\text { resilience } \\
\text { funding }\end{array}$ & impl & $\begin{array}{l}\text { base of type } \\
\text { schedule }\end{array}$ & FRM:SG1.SP1 \\
\hline $\begin{array}{l}\text { FRM- } \\
\text { M4 }\end{array}$ & $\begin{array}{l}\text { difference in planned versus actual fund- } \\
\text { ing for the ORM system }\end{array}$ & $\begin{array}{l}\text { resilience } \\
\text { funding }\end{array}$ & $\begin{array}{l}\text { impl; pos- } \\
\text { sibly effec- } \\
\text { tiveness }\end{array}$ & derived & FRM:SG1.SP1 \\
\hline $\begin{array}{l}\text { FRM- } \\
\text { M5 }\end{array}$ & $\begin{array}{l}\text { elapsed time since responsibility and ac- } \\
\text { countability for resilience budgeting, fund- } \\
\text { ing, and accounting activities were re- } \\
\text { viewed }\end{array}$ & $\begin{array}{l}\text { resilience } \\
\text { financial } \\
\text { structure }\end{array}$ & impl & $\begin{array}{l}\text { base of type } \\
\text { schedule }\end{array}$ & FRM:SG1.SP2 \\
\hline $\begin{array}{l}\text { FRM- } \\
\text { M6 }\end{array}$ & $\begin{array}{l}\text { percentage of resilience activities for which } \\
\text { historical financial cost data is used as the } \\
\text { basis for developing funding requirements }\end{array}$ & $\begin{array}{l}\text { resilience } \\
\text { funding }\end{array}$ & impl & derived & FRM:SG2.SP1 \\
\hline $\begin{array}{l}\text { FRM- } \\
\text { M7 }\end{array}$ & $\begin{array}{l}\text { percentage of resilience funding assump- } \\
\text { tions that have been validated by compari- } \\
\text { son to resilience requirements }\end{array}$ & $\begin{array}{l}\text { resilience } \\
\text { funding }\end{array}$ & impl & derived & FRM:SG2.SP1 \\
\hline $\begin{array}{l}\text { FRM- } \\
\text { M8 }\end{array}$ & cost of resilience (COR) calculations & $\begin{array}{l}\text { resilience } \\
\text { cost }\end{array}$ & impl & derived & $\begin{array}{l}\text { FRM:SG4.SP1 } \\
\text { FRM:SG4.SP2 } \\
\text { FRM:SG5.SP2 }\end{array}$ \\
\hline $\begin{array}{l}\text { FRM- } \\
\text { M9 }\end{array}$ & $\begin{array}{l}\text { return on resilience investment (RORI) } \\
\text { calculations }\end{array}$ & $\begin{array}{l}\text { resilience } \\
\text { cost; resi- } \\
\text { lience benefit }\end{array}$ & impl & derived & $\begin{array}{l}\text { FRM:SG4.SP1 } \\
\text { FRM:SG4.SP2 } \\
\text { FRM:SG5.SP2 }\end{array}$ \\
\hline $\begin{array}{l}\text { FRM- } \\
\text { M10 }\end{array}$ & $\begin{array}{l}\text { percentage of resilience costs that are } \\
\text { included as part of standard costs for ser- } \\
\text { vices and products (chargebacks) }\end{array}$ & $\begin{array}{l}\text { resilience } \\
\text { cost }\end{array}$ & impl & derived & FRM:SG5.SP3 \\
\hline $\begin{array}{l}\text { FRM- } \\
\text { M11 }\end{array}$ & $\begin{array}{l}\text { percentage of assets and services for } \\
\text { which optimization }{ }^{32} \text { calculations have } \\
\text { been performed }\end{array}$ & $\begin{array}{l}\text { resilience } \\
\text { cost; resi- } \\
\text { lience benefit }\end{array}$ & impl & derived & FRM:SG5.SP1 \\
\hline $\begin{array}{l}\text { FRM- } \\
\text { M12 }\end{array}$ & $\begin{array}{l}\text { percentage of optimization opportunities } \\
\text { for which no action has been taken }\end{array}$ & $\begin{array}{l}\text { resilience } \\
\text { cost; resi- } \\
\text { lience benefit }\end{array}$ & impl & derived & $\begin{array}{l}\text { FRM:SG5.SP1 } \\
\text { FRM:SG5.SP2 }\end{array}$ \\
\hline $\begin{array}{l}\text { FRM- } \\
\text { M13 }\end{array}$ & $\begin{array}{l}\text { percentage of resilience activities with } \\
\text { required budgets assigned, allocated, and } \\
\text { applied, organized by organizational unit, } \\
\text { project, asset, and service or other mea- } \\
\text { ningful categorization scheme }\end{array}$ & $\begin{array}{l}\text { resilience } \\
\text { budgeting }\end{array}$ & impl & derived & FRM:SG2.SP2 \\
\hline $\begin{array}{l}\text { FRM- } \\
\text { M14 }\end{array}$ & $\begin{array}{l}\text { elapsed time since resilience budgets } \\
\text { were reviewed and updated }\end{array}$ & $\begin{array}{l}\text { resilience } \\
\text { budgeting }\end{array}$ & impl & $\begin{array}{l}\text { base of type } \\
\text { schedule }\end{array}$ & $\begin{array}{l}\text { FRM:SG2.SP2 } \\
\text { FRM:SG4.SP1 }\end{array}$ \\
\hline
\end{tabular}

32 The costs of attaining and sustaining an adequate level of operational resilience for an asset or service must be optimized against the value of the asset or service in order to rationalize and maximize the organization's investment in resilience. 


\begin{tabular}{|c|c|c|c|c|c|}
\hline ID & Measure & $\begin{array}{l}\text { Type of } \\
\text { Information }\end{array}$ & $\begin{array}{l}\text { Measure } \\
\text { Type }\end{array}$ & $\begin{array}{l}\text { Base or } \\
\text { Derived }\end{array}$ & $\begin{array}{l}\text { Applicable } \\
\text { SG.SP }\end{array}$ \\
\hline $\begin{array}{l}\text { FRM- } \\
\text { M15 }\end{array}$ & $\begin{array}{l}\text { elapsed time since resilience budgets } \\
\text { were reviewed to confirm their adequacy } \\
\text { to meet resilience performance measures }\end{array}$ & $\begin{array}{l}\text { resilience } \\
\text { budgeting }\end{array}$ & impl & $\begin{array}{l}\text { base of type } \\
\text { schedule }\end{array}$ & FRM:SG2.SP2 \\
\hline $\begin{array}{l}\text { FRM- } \\
\text { M16 }\end{array}$ & $\begin{array}{l}\text { percentage of resilience activities subject } \\
\text { to off-cycle or off-budget funding requests }\end{array}$ & $\begin{array}{l}\text { resilience } \\
\text { budgeting }\end{array}$ & $\begin{array}{l}\text { effective- } \\
\text { ness }\end{array}$ & derived & FRM:SG3.SP1 \\
\hline $\begin{array}{l}\text { FRM- } \\
\text { M17 }\end{array}$ & $\begin{array}{l}\text { percentage of resilience activities tracking } \\
\text { to planned budgets }\end{array}$ & $\begin{array}{l}\text { resilience } \\
\text { budgeting }\end{array}$ & $\begin{array}{l}\text { effective- } \\
\text { ness }\end{array}$ & derived & FRM:SG3.SP1 \\
\hline $\begin{array}{l}\text { FRM- } \\
\text { M18 }\end{array}$ & $\begin{array}{l}\text { difference in planned versus actual cost for } \\
\text { the ORM system }\end{array}$ & $\begin{array}{l}\text { resilience } \\
\text { cost }\end{array}$ & $\begin{array}{l}\text { effective- } \\
\text { ness }\end{array}$ & derived & FRM:SG4.SP1 \\
\hline $\begin{array}{l}\text { FRM- } \\
\text { M19 }\end{array}$ & $\begin{array}{l}\text { percentage of resilience activities with } \\
\text { budget variances outside of established } \\
\text { thresholds for which resolution plans have } \\
\text { been developed to reduce or eliminate } \\
\text { these variances }\end{array}$ & $\begin{array}{l}\text { resilience } \\
\text { budgeting }\end{array}$ & impl & derived & $\begin{array}{l}\text { FRM:SG4.SP1 } \\
\text { FRM:SG4.SP2 }\end{array}$ \\
\hline $\begin{array}{l}\text { FRM- } \\
\text { M20 }\end{array}$ & $\begin{array}{l}\text { percentage of financial exceptions re- } \\
\text { ported to oversight managers and commit- } \\
\text { tees }\end{array}$ & $\begin{array}{l}\text { resilience } \\
\text { budgeting }\end{array}$ & impl & derived & FRM:SG4.SP2 \\
\hline $\begin{array}{l}\text { FRM- } \\
\text { M21 }\end{array}$ & $\begin{array}{l}\text { percentage of resilience activities without } \\
\text { required budget allocations for which gap } \\
\text { and risk analysis has been performed }\end{array}$ & $\begin{array}{l}\text { resilience } \\
\text { budgeting; } \\
\text { risk identifi- } \\
\text { cation }\end{array}$ & impl & derived & FRM:SG2.SP3 \\
\hline $\begin{array}{l}\text { FRM- } \\
\text { M22 }\end{array}$ & $\begin{array}{l}\text { number of budget shorfall risks referred to } \\
\text { the risk management process }\end{array}$ & $\begin{array}{l}\text { risk identifi- } \\
\text { cation }\end{array}$ & impl & $\begin{array}{l}\text { base of type } \\
\text { count }\end{array}$ & FRM:SG2.SP3 \\
\hline
\end{tabular}

\section{Human Resource Management (HRM)}

The purpose of Human Resource Management is to manage the employment life cycle and performance of staff in a manner that contributes to the organization's ability to manage operational resilience.

\section{Summary of Specific Goals and Practices}

HRM:SG1 Establish Resource Needs

HRM:SG1.SP1 Establish Baseline Competencies

HRM:SG1.SP2 Inventory Skills and Identify Gaps

HRM:SG1.SP3 Address Skill Deficiencies

HRM:SG2 Manage Staff Acquisition

HRM:SG2.SP1 Verify Suitability of Candidate Staff

HRM:SG2.SP2 Establish Terms and Conditions of Employment

HRM:SG3 Manage Staff Performance

HRM:SG3.SP1 Establish Resilience as a Job Responsibility

HRM:SG3.SP2 Establish Resilience Performance Goals and Objectives

HRM:SG3.SP3 Measure and Assess Performance

HRM:SG3.SP4 Establish Disciplinary Process

HRM:SG4 Manage Changes to Employment Status

HRM:SG4.SP1 Manage Impact of Position Changes 
HRM:SG4.SP2 Manage Access to Assets

HRM:SG4.SP3 Manage Involuntary Terminations

\section{Measures}

\begin{tabular}{|c|c|c|c|c|c|}
\hline ID & Measure & $\begin{array}{l}\text { Type of } \\
\text { Information }\end{array}$ & $\begin{array}{l}\text { Measure } \\
\text { Type }\end{array}$ & $\begin{array}{l}\text { Base or } \\
\text { Derived }\end{array}$ & $\begin{array}{l}\text { Applicable } \\
\text { SG.SP }\end{array}$ \\
\hline $\begin{array}{l}\text { HRM- } \\
\text { M1 }\end{array}$ & $\begin{array}{l}\text { percentage of job descriptions in which } \\
\text { resilience competencies and skills are iden- } \\
\text { tified }\end{array}$ & $\begin{array}{l}\text { resilience skill } \\
\text { needs }\end{array}$ & impl & derived & $\begin{array}{l}\text { HRM:SG1. } \\
\text { SP1 }\end{array}$ \\
\hline $\begin{array}{l}\text { HRM- } \\
\text { M2 }\end{array}$ & $\begin{array}{l}\text { percentage of job descriptions with docu- } \\
\text { mented terms and conditions }\end{array}$ & job descriptions & impl & derived & $\begin{array}{l}\text { HRM:SG2. } \\
\text { SP2 }\end{array}$ \\
\hline $\begin{array}{l}\text { HRM- } \\
\text { M3 }\end{array}$ & $\begin{array}{l}\text { percentage of job descriptions with docu- } \\
\text { mented resilience obligations }\end{array}$ & job descriptions & impl & derived & $\begin{array}{l}\text { HRM:SG2. } \\
\text { SP2HRM:S } \\
\text { G3.SP1 }\end{array}$ \\
\hline $\begin{array}{l}\text { HRM- } \\
\text { M4 }\end{array}$ & $\begin{array}{l}\text { percentage of vital staff with resilience skill } \\
\text { deficiencies }\end{array}$ & $\begin{array}{l}\text { resilience skill } \\
\text { needs }\end{array}$ & impl & derived & $\begin{array}{l}\text { HRM:SG1. } \\
\text { SP2 }\end{array}$ \\
\hline $\begin{array}{l}\text { HRM- } \\
\text { M5 }\end{array}$ & $\begin{array}{l}\text { cost required to address resilience skill } \\
\text { gaps }\end{array}$ & $\begin{array}{l}\text { resilience skill } \\
\text { needs; resilience } \\
\text { cost }\end{array}$ & impl & $\begin{array}{l}\text { base of } \\
\text { type cost }\end{array}$ & $\begin{array}{l}\text { HRM:SG1. } \\
\text { SP3 }\end{array}$ \\
\hline $\begin{array}{l}\text { HRM- } \\
\text { M6 }\end{array}$ & $\begin{array}{l}\text { schedule required to address resilience skill } \\
\text { gaps }\end{array}$ & $\begin{array}{l}\text { resilience skill } \\
\text { needs }\end{array}$ & impl & $\begin{array}{l}\text { base of } \\
\text { type sche- } \\
\text { dule }\end{array}$ & $\begin{array}{l}\text { HRM:SG1. } \\
\text { SP3 }\end{array}$ \\
\hline $\begin{array}{l}\text { HRM- } \\
\text { M7 }\end{array}$ & $\begin{array}{l}\text { effort required to address resilience skill } \\
\text { gaps }\end{array}$ & $\begin{array}{l}\text { resilience skill } \\
\text { needs }\end{array}$ & impl & $\begin{array}{l}\text { base of } \\
\text { type effort }\end{array}$ & $\begin{array}{l}\text { HRM:SG1. } \\
\text { SP3 }\end{array}$ \\
\hline $\begin{array}{l}\text { HRM- } \\
\text { M8 }\end{array}$ & $\begin{array}{l}\text { percentage of resilience training delivered } \\
\text { as scheduled }\end{array}$ & $\begin{array}{l}\text { resilience skill } \\
\text { needs; resilience } \\
\text { training }\end{array}$ & impl & derived & $\begin{array}{l}\text { HRM:SG1. } \\
\text { SP3 }\end{array}$ \\
\hline $\begin{array}{l}\text { HRM- } \\
\text { M9 }\end{array}$ & $\begin{array}{l}\text { rate of changes to the resilience skills in- } \\
\text { ventory }\end{array}$ & skills inventory & impl & derived & $\begin{array}{l}\text { HRM:SG1. } \\
\text { SP2 }\end{array}$ \\
\hline $\begin{array}{l}\text { HRM- } \\
\text { M10 }\end{array}$ & $\begin{array}{l}\text { elapsed time since the resilience skills in- } \\
\text { ventory was compared to baseline resi- } \\
\text { lience competencies and skills }\end{array}$ & skills inventory & impl & $\begin{array}{l}\text { base of } \\
\text { type sche- } \\
\text { dule }\end{array}$ & $\begin{array}{l}\text { HRM:SG1. } \\
\text { SP2 }\end{array}$ \\
\hline $\begin{array}{l}\text { HRM- } \\
\text { M11 }\end{array}$ & $\begin{array}{l}\text { percentage of acquired vital staff that have } \\
\text { met pre-employment verification criteria } \\
\text { (baseline and job-specific) }\end{array}$ & staff suitability & impl & derived & $\begin{array}{l}\text { HRM:SG2. } \\
\text { SP1 }\end{array}$ \\
\hline $\begin{array}{l}\text { HRM- } \\
\text { M12 }\end{array}$ & $\begin{array}{l}\text { percentage of acquired staff that have } \\
\text { signed agreements to acknowledge and } \\
\text { consent to employment terms and condi- } \\
\text { tions }\end{array}$ & $\begin{array}{l}\text { terms and condi- } \\
\text { tions of employ- } \\
\text { ment }\end{array}$ & impl & derived & $\begin{array}{l}\text { HRM:SG2. } \\
\text { SP2 }\end{array}$ \\
\hline $\begin{array}{l}\text { HRM- } \\
\text { M13 }\end{array}$ & $\begin{array}{l}\text { percentage of confidentiality and non- } \\
\text { compete agreements executed for people in } \\
\text { sensitive positions }\end{array}$ & $\begin{array}{l}\text { terms and condi- } \\
\text { tions of employ- } \\
\text { ment }\end{array}$ & impl & derived & $\begin{array}{l}\text { HRM:SG2. } \\
\text { SP2 }\end{array}$ \\
\hline $\begin{array}{l}\text { HRM- } \\
\text { M14 }\end{array}$ & $\begin{array}{l}\text { number of performance reviews performed } \\
\text { (by type) }\end{array}$ & $\begin{array}{l}\text { performance } \\
\text { evaluation }\end{array}$ & impl & $\begin{array}{l}\text { base of } \\
\text { type count }\end{array}$ & $\begin{array}{l}\text { HRM:SG3. } \\
\text { SP3 }\end{array}$ \\
\hline $\begin{array}{l}\text { HRM- } \\
\text { M15 }\end{array}$ & $\begin{array}{l}\text { percentage of staff that have resilience } \\
\text { performance goals and objectives }\end{array}$ & $\begin{array}{l}\text { performance } \\
\text { evaluation }\end{array}$ & impl & derived & $\begin{array}{l}\text { HRM:SG3. } \\
\text { SP2 }\end{array}$ \\
\hline $\begin{array}{l}\text { HRM- } \\
\text { M16 }\end{array}$ & $\begin{array}{l}\text { percentage of staff that have met/not met } \\
\text { their resilience performance goals and ob- } \\
\text { jectives }\end{array}$ & $\begin{array}{l}\text { performance } \\
\text { evaluation }\end{array}$ & impl & derived & $\begin{array}{l}\text { HRM:SG3. } \\
\text { SP2 }\end{array}$ \\
\hline $\begin{array}{l}\text { HRM- } \\
\text { M17 }\end{array}$ & $\begin{array}{l}\text { number of infractions referred to the inci- } \\
\text { dent management process }\end{array}$ & disciplinary action & impl & $\begin{array}{l}\text { base of } \\
\text { type count }\end{array}$ & $\begin{array}{l}\text { HRM:SG3. } \\
\text { SP4 }\end{array}$ \\
\hline $\begin{array}{l}\text { HRM- } \\
\text { M18 }\end{array}$ & $\begin{array}{l}\text { number of infractions requiring coordination } \\
\text { with public authorities }\end{array}$ & disciplinary action & impl & $\begin{array}{l}\text { base of } \\
\text { type count }\end{array}$ & $\begin{array}{l}\text { HRM:SG3. } \\
\text { SP4 }\end{array}$ \\
\hline
\end{tabular}




\begin{tabular}{|c|c|c|c|c|c|}
\hline ID & Measure & $\begin{array}{l}\text { Type of } \\
\text { Information }\end{array}$ & $\begin{array}{l}\text { Measure } \\
\text { Type }\end{array}$ & $\begin{array}{l}\text { Base or } \\
\text { Derived }\end{array}$ & $\begin{array}{l}\text { Applicable } \\
\text { SG.SP }\end{array}$ \\
\hline $\begin{array}{l}\text { HRM- } \\
\text { M19 }\end{array}$ & $\begin{array}{l}\text { number of violations of resilience policies } \\
\text { subject to disciplinary action }\end{array}$ & $\begin{array}{l}\text { disciplinary action; } \\
\text { resilience policy } \\
\text { compliance }\end{array}$ & impl & $\begin{array}{l}\text { base of } \\
\text { type count }\end{array}$ & $\begin{array}{l}\text { HRM:SG3. } \\
\text { SP4 }\end{array}$ \\
\hline $\begin{array}{l}\text { HRM- } \\
\text { M20 }\end{array}$ & $\begin{array}{l}\text { elapsed time since measures of resilience } \\
\text { policy compliance were collected and re- } \\
\text { viewed }\end{array}$ & $\begin{array}{l}\text { resilience policy } \\
\text { compliance }\end{array}$ & impl & $\begin{array}{l}\text { base of } \\
\text { type sche- } \\
\text { dule }\end{array}$ & $\begin{array}{l}\text { HRM:SG3. } \\
\text { SP4 }\end{array}$ \\
\hline $\begin{array}{l}\text { HRM- } \\
\text { M21 }\end{array}$ & $\begin{array}{l}\text { number of skill gaps referred to the risk } \\
\text { management process }\end{array}$ & risk identification & impl & $\begin{array}{l}\text { base of } \\
\text { type count }\end{array}$ & $\begin{array}{l}\text { HRM:SG1. } \\
\text { SP3 }\end{array}$ \\
\hline $\begin{array}{l}\text { HRM- } \\
\text { M22 }\end{array}$ & $\begin{array}{l}\text { percentage of departing staff (from a posi- } \\
\text { tion, from the organization) that participate } \\
\text { in an exit interview }\end{array}$ & $\begin{array}{l}\text { changes of em- } \\
\text { ployment status }\end{array}$ & impl & derived & $\begin{array}{l}\text { HRM;SG4. } \\
\text { SP1 }\end{array}$ \\
\hline $\begin{array}{l}\text { HRM- } \\
\text { M23 }\end{array}$ & $\begin{array}{l}\text { percentage of departing staff (from a posi- } \\
\text { tion, from the organization) that have re- } \\
\text { turned all organizational assets, property, } \\
\text { and information }\end{array}$ & $\begin{array}{l}\text { changes of em- } \\
\text { ployment status }\end{array}$ & impl & derived & $\begin{array}{l}\text { HRM;SG4. } \\
\text { SP2 }\end{array}$ \\
\hline $\begin{array}{l}\text { HRM- } \\
\text { M24 }\end{array}$ & $\begin{array}{l}\text { percentage of departing staff (from a posi- } \\
\text { tion, from the organization) whose access } \\
\text { rights have been discontinued as scheduled }\end{array}$ & $\begin{array}{l}\text { changes of em- } \\
\text { ployment status }\end{array}$ & impl & derived & $\begin{array}{l}\text { HRM;SG4. } \\
\text { SP2 }\end{array}$ \\
\hline $\begin{array}{l}\text { HRM- } \\
\text { M25 }\end{array}$ & $\begin{array}{l}\text { percentage of involuntary terminations that } \\
\text { are processed in accordance with estab- } \\
\text { lished criteria and procedures }\end{array}$ & $\begin{array}{l}\text { changes of em- } \\
\text { ployment status }\end{array}$ & impl & derived & $\begin{array}{l}\text { HRM;SG4. } \\
\text { SP3 }\end{array}$ \\
\hline
\end{tabular}

\section{Identity Management (ID)}

The purpose of Identity Management is to create, maintain, and deactivate identities that may need some level of trusted access to organizational assets and to manage their associated attributes.

\section{Summary of Specific Goals and Practices}

ID:SG1 Establish Identities

ID:SG1.SP1 Create Identities

ID:SG1.SP2 Establish Identity Community

ID:SG1.SP3 Assign Roles to Identities

ID:SG2 Manage Identities

ID:SG2.SP1 Monitor and Manage Identity Changes

ID:SG2.SP2 Periodically Review and Maintain Identities

ID:SG2.SP3 Correct Inconsistencies

ID:SG2.SP4 Deprovision Identities

\section{Measures}

\begin{tabular}{|l|l|l|l|l|l|}
\hline ID & Measure & $\begin{array}{l}\text { Type of } \\
\text { Information }\end{array}$ & $\begin{array}{l}\text { Measure } \\
\text { Type }\end{array}$ & $\begin{array}{l}\text { Base or } \\
\text { Derived }\end{array}$ & $\begin{array}{l}\text { Applicable } \\
\text { SG.SP }\end{array}$ \\
\hline ID-M1 & $\begin{array}{l}\text { elapsed time from identity request to grant- } \\
\text { ing of identity credentials }\end{array}$ & identity requests & $\begin{array}{l}\text { effective- } \\
\text { ness }\end{array}$ & $\begin{array}{l}\text { base of } \\
\text { type } \\
\text { schedule }\end{array}$ & ID:SG1.SP1 \\
\hline ID-M2 & $\begin{array}{l}\text { percentage of identity requests denied } \\
\text { (based on policy) }\end{array}$ & identity requests & impl & derived & ID:SG1.SP1 \\
\hline
\end{tabular}




\begin{tabular}{|c|c|c|c|c|c|}
\hline ID & Measure & $\begin{array}{l}\text { Type of } \\
\text { Information }\end{array}$ & $\begin{array}{l}\text { Measure } \\
\text { Type }\end{array}$ & $\begin{array}{l}\text { Base or } \\
\text { Derived }\end{array}$ & $\begin{array}{l}\text { Applicable } \\
\text { SG.SP }\end{array}$ \\
\hline ID-M3 & $\begin{array}{l}\text { percentage of identity requests approved } \\
\text { that, on further investigation, should have } \\
\text { been denied based on, for example, a mis- } \\
\text { match with designated roles }\end{array}$ & identity requests & $\begin{array}{l}\text { effective- } \\
\text { ness }\end{array}$ & derived & ID:SG2.SP2 \\
\hline ID-M4 & $\begin{array}{l}\text { percentage of identity requests that dupli- } \\
\text { cate previous or current requests }\end{array}$ & identity requests & impl & derived & ID:SG1.SP1 \\
\hline ID-M5 & $\begin{array}{l}\text { percentage of identities for which roles have } \\
\text { been authorized and justified by identity } \\
\text { owners }\end{array}$ & identity roles & impl & derived & ID:SG1.SP3 \\
\hline ID-M6 & $\begin{array}{l}\text { rate of change requests to current identity } \\
\text { profiles }\end{array}$ & identity profiles & impl & derived & ID:SG2.SP1 \\
\hline ID-M7 & $\begin{array}{l}\text { number of inconsistencies between identity } \\
\text { profiles and their associated persons, ob- } \\
\text { jects, and entities }\end{array}$ & $\begin{array}{l}\text { identity profiles; } \\
\text { identity commu- } \\
\text { nity }\end{array}$ & $\begin{array}{l}\text { effective- } \\
\text { ness }\end{array}$ & $\begin{array}{l}\text { base of } \\
\text { type } \\
\text { count }\end{array}$ & $\begin{array}{l}\text { ID:SG1.SP1 } \\
\text { ID:SG2.SP1 }\end{array}$ \\
\hline ID-M8 & $\begin{array}{l}\text { percentage of identity profiles that are inac- } \\
\text { curate }\end{array}$ & identity profiles & $\begin{array}{l}\text { effective- } \\
\text { ness }\end{array}$ & derived & ID:SG2.SP2 \\
\hline ID-M9 & $\begin{array}{l}\text { percentage of identity profiles that are va- } \\
\text { cant or invalid }\end{array}$ & identity profiles & $\begin{array}{l}\text { effective- } \\
\text { ness }\end{array}$ & derived & ID:SG2.SP2 \\
\hline ID-M10 & $\begin{array}{l}\text { percentage of identity profiles that are re- } \\
\text { dundant }\end{array}$ & identity profiles & $\begin{array}{l}\text { effective- } \\
\text { ness }\end{array}$ & derived & ID:SG2.SP2 \\
\hline ID-M11 & $\begin{array}{l}\text { percentage of identity community inconsis- } \\
\text { tencies for which corrective action is pend- } \\
\text { ing beyond schedule }\end{array}$ & $\begin{array}{l}\text { identity profiles; } \\
\text { identity commu- } \\
\text { nity }\end{array}$ & impl & derived & $\begin{array}{l}\text { ID:SG2.SP2 } \\
\text { ID:SG2.SP3 }\end{array}$ \\
\hline ID-M12 & $\begin{array}{l}\text { percentage of identities belonging to exter- } \\
\text { nal entities }\end{array}$ & $\begin{array}{l}\text { identity commu- } \\
\text { nity }\end{array}$ & impl & derived & ID:SG1.SP1 \\
\hline ID-M13 & $\begin{array}{l}\text { percentage of deprovisioned identities } \\
\text { whose deprovisioning is pending beyond } \\
\text { schedule }\end{array}$ & deprovisioning & impl & derived & ID:SG2.SP4 \\
\hline ID-M14 & $\begin{array}{l}\text { number of incidents involving the identity } \\
\text { repository }\end{array}$ & $\begin{array}{l}\text { identity reposito- } \\
\text { ry; incident anal- } \\
\text { ysis }\end{array}$ & impl & $\begin{array}{l}\text { base of } \\
\text { type } \\
\text { count }\end{array}$ & ID:SG1.SP2 \\
\hline ID-M15 & $\begin{array}{l}\text { number of incidents involving the identity } \\
\text { repository for which resolution is pending } \\
\text { beyond schedule }\end{array}$ & $\begin{array}{l}\text { identity reposito- } \\
\text { ry; incident anal- } \\
\text { ysis }\end{array}$ & impl & $\begin{array}{l}\text { base of } \\
\text { type } \\
\text { defect }\end{array}$ & $\begin{array}{l}\text { ID:SG1.SP2 } \\
\text { IMC:SG4.SP } \\
2\end{array}$ \\
\hline ID-M16 & $\begin{array}{l}\text { number of identity-related risks referred to } \\
\text { the risk management process }\end{array}$ & risk identification & impl & $\begin{array}{l}\text { base of } \\
\text { type } \\
\text { count }\end{array}$ & ID:SG2.SP3 \\
\hline
\end{tabular}

\section{Incident Management and Control (IMC)}

The purpose of Incident Management and Control is to establish processes to identify and analyze events, detect incidents, and determine an appropriate organizational response.

\section{Summary of Specific Goals and Practices}

IMC:SG1 Establish the Incident Management and Control Process

IMC:SG1.SP1 Plan for Incident Management

IMC:SG1.SP2 Assign Staff to the Incident Management Plan

\section{IMC:SG2 Detect Events}

IMC:SG2.SP1 Detect and Report Events

IMC:SG2.SP2 Log and Track Events 
IMC:SG2.SP3 Collect, Document, and Preserve Event Evidence

IMC:SG2.SP4 Analyze and Triage Events

IMC:SG3 Declare Incidents

IMC:SG3.SP1 Define and Maintain Incident Declaration Criteria

IMC:SG3.SP2 Analyze Incidents

IMC:SG4 Respond to and Recover from Incidents

IMC:SG4.SP1 Escalate Incidents

IMC:SG4.SP2 Develop Incident Response

IMC:SG4.SP3 Communicate Incidents

IMC:SG4.SP4 Close Incidents

IMC:SG5 Establish Incident Learning

IMC:SG5.SP1 Perform Post-Incident Review

IMC:SG5.SP2 Integrate with the Problem Management Process

IMC:SG5.SP3 Translate Experience to Strategy

\section{Measures}

\begin{tabular}{|c|c|c|c|c|c|}
\hline ID & Measure & $\begin{array}{l}\text { Type of } \\
\text { Information }\end{array}$ & $\begin{array}{l}\text { Measure } \\
\text { Type }\end{array}$ & $\begin{array}{l}\text { Base or } \\
\text { Derived }\end{array}$ & $\begin{array}{l}\text { Applicable } \\
\text { SG.SP }\end{array}$ \\
\hline $\begin{array}{l}\text { IMC- } \\
\text { M1 }\end{array}$ & $\begin{array}{l}\text { percentage of coverage of IM plan (extent to } \\
\text { which IM management plan includes all organi- } \\
\text { zational units and functions that require cover- } \\
\text { age; aka IM plan scope) }\end{array}$ & IM planning & $\begin{array}{l}\text { impl; possi- } \\
\text { bly effec- } \\
\text { tiveness }\end{array}$ & derived & IMC:SG1.SP1 \\
\hline $\begin{array}{l}\text { IMC- } \\
\text { M2 }\end{array}$ & $\begin{array}{l}\text { percentage of IM roles/responsibilities assigned } \\
\text { to staff roles/members (extent to which IM plan } \\
\text { roles and tasks are assigned to specific staff } \\
\text { roles/members) }\end{array}$ & IM roles & impl & derived & $\begin{array}{l}\text { IMC:SG1.SP2 } \\
\text { IMC:SG2.SP1 }\end{array}$ \\
\hline $\begin{array}{l}\text { IMC- } \\
\text { M3 }\end{array}$ & $\begin{array}{l}\text { percentage of staff who have not been trained } \\
\text { on their roles and responsibilities as defined in } \\
\text { IM plans }\end{array}$ & IM training & impl & derived & IMC:SG1.SP2 \\
\hline $\begin{array}{l}\text { IMC- } \\
\text { M4 }\end{array}$ & $\begin{array}{l}\text { percentage of staff (managers, users) who } \\
\text { have not completed training and awareness to } \\
\text { identify anomalies and report them in the re- } \\
\text { quired timeframe (initial, refresher) }\end{array}$ & IM training & impl & derived & IMC:SG2.SP1 \\
\hline $\begin{array}{l}\text { IMC- } \\
\text { M5 }\end{array}$ & $\begin{array}{l}\text { percentage of events triaged (events reported } \\
\text { vs. events analyzed) }\end{array}$ & $\begin{array}{l}\text { event analy- } \\
\text { sis }\end{array}$ & impl & derived & IMC:SG2.SP4 \\
\hline $\begin{array}{l}\text { IMC- } \\
\text { M6 }\end{array}$ & $\begin{array}{l}\text { percentage of events that are stalled or await- } \\
\text { ing activity beyond threshold }\end{array}$ & $\begin{array}{l}\text { event analy- } \\
\text { sis }\end{array}$ & $\begin{array}{l}\text { impl; possi- } \\
\text { bly effec- } \\
\text { tiveness }\end{array}$ & derived & $\begin{array}{l}\text { IMC:SG2.SP1 } \\
\text { IMC:SG2.SP2 }\end{array}$ \\
\hline $\begin{array}{l}\text { IMC- } \\
\text { M7 }\end{array}$ & $\begin{array}{l}\text { percentage of events whose documentation } \\
\text { does not meet rules, laws, regulations, policies, } \\
\text { or other requirements for forensic purposes }\end{array}$ & $\begin{array}{l}\text { event analy- } \\
\text { sis }\end{array}$ & impl & derived & IMC:SG2.SP3 \\
\hline $\begin{array}{l}\text { IMC- } \\
\text { M8 }\end{array}$ & percentage of events without a disposition & $\begin{array}{l}\text { event analy- } \\
\text { sis }\end{array}$ & impl & derived & IMC:SG2.SP4 \\
\hline $\begin{array}{l}\text { IMC- } \\
\text { M9 }\end{array}$ & $\begin{array}{l}\text { percentage of events open beyond scheduled } \\
\text { threshold (such as specified number of days for } \\
\text { closure) }\end{array}$ & $\begin{array}{l}\text { event analy- } \\
\text { sis }\end{array}$ & impl & derived & IMC:SG2.SP4 \\
\hline $\begin{array}{l}\text { IMC- } \\
\text { M10 }\end{array}$ & $\begin{array}{l}\text { mean, median time to close an event, catego- } \\
\text { rized in some meaningful manner }\end{array}$ & $\begin{array}{l}\text { event analy- } \\
\text { sis }\end{array}$ & $\begin{array}{l}\text { impl; possi- } \\
\text { bly effec- } \\
\text { tiveness }\end{array}$ & derived & IMC:SG2.SP4 \\
\hline
\end{tabular}




\begin{tabular}{|c|c|c|c|c|c|}
\hline ID & Measure & $\begin{array}{l}\text { Type of } \\
\text { Information }\end{array}$ & $\begin{array}{l}\text { Measure } \\
\text { Type }\end{array}$ & $\begin{array}{l}\text { Base or } \\
\text { Derived }\end{array}$ & $\begin{array}{l}\text { Applicable } \\
\text { SG.SP }\end{array}$ \\
\hline $\begin{array}{l}\text { IMC- } \\
\text { M11 }\end{array}$ & $\begin{array}{l}\text { percentage change in the number of logged } \\
\text { events }\end{array}$ & $\begin{array}{l}\text { event analy- } \\
\text { sis }\end{array}$ & $\begin{array}{l}\text { impl; possi- } \\
\text { bly effec- } \\
\text { tiveness }\end{array}$ & derived & IMC:SG2.SP2 \\
\hline $\begin{array}{l}\text { IMC- } \\
\text { M12 }\end{array}$ & $\begin{array}{l}\text { percentage of events that recur and result in } \\
\text { declared incidents }\end{array}$ & $\begin{array}{l}\text { incident } \\
\text { analysis }\end{array}$ & $\begin{array}{l}\text { impl; possi- } \\
\text { bly effec- } \\
\text { tiveness }\end{array}$ & derived & $\begin{array}{l}\text { IMC:SG3.SP2 } \\
\text { IMC:SG5.SP1 }\end{array}$ \\
\hline $\begin{array}{l}\text { IMC- } \\
\text { M13 }\end{array}$ & $\begin{array}{l}\text { percentage of events (or sets of related events) } \\
\text { declared as incidents }\end{array}$ & $\begin{array}{l}\text { incident } \\
\text { analysis }\end{array}$ & impl & derived & $\begin{array}{l}\text { IMC:SG3.SP2 } \\
\text { IMC:SG5.SP1 }\end{array}$ \\
\hline $\begin{array}{l}\text { IMC- } \\
\text { M14 }\end{array}$ & $\begin{array}{l}\text { percentage of events declared as incidents that } \\
\text { do not match the current incident declaration } \\
\text { criteria }\end{array}$ & $\begin{array}{l}\text { incident } \\
\text { analysis }\end{array}$ & impl & derived & IMC:SG3.SP1 \\
\hline $\begin{array}{l}\text { IMC- } \\
\text { M15 }\end{array}$ & number of incidents by incident type & $\begin{array}{l}\text { incident } \\
\text { analysis }\end{array}$ & impl & $\begin{array}{l}\text { base of } \\
\text { type } \\
\text { count }\end{array}$ & IMC:SG3.SP2 \\
\hline $\begin{array}{l}\text { IMC- } \\
\text { M16 }\end{array}$ & $\begin{array}{l}\text { percentage of incidents that have been de- } \\
\text { clared but not closed }\end{array}$ & $\begin{array}{l}\text { incident } \\
\text { analysis }\end{array}$ & impl & derived & $\begin{array}{l}\text { IMC:SG3.SP2 } \\
\text { IMC:SG4.SP4 }\end{array}$ \\
\hline $\begin{array}{l}\text { IMC- } \\
\text { M17 }\end{array}$ & $\begin{array}{l}\text { percentage of incidents that exploited existing } \\
\text { vulnerabilities with known solutions, patches, or } \\
\text { workarounds }\end{array}$ & $\begin{array}{l}\text { incident } \\
\text { analysis }\end{array}$ & impl & derived & $\begin{array}{l}\text { IMC:SG3.SP2 } \\
\text { IMC:SG5.SP1 }\end{array}$ \\
\hline $\begin{array}{l}\text { IMC- } \\
\text { M18 }\end{array}$ & $\begin{array}{l}\text { percentage of operational time that services } \\
\text { and assets were unavailable (as seen by users } \\
\text { and customers) due to incidents }\end{array}$ & $\begin{array}{l}\text { incident } \\
\text { analysis }\end{array}$ & $\begin{array}{l}\text { effective- } \\
\text { ness }\end{array}$ & derived & IMC:SG5.SP1 \\
\hline $\begin{array}{l}\text { IMC- } \\
\text { M19 }\end{array}$ & $\begin{array}{l}\text { number of incidents by incident type and im- } \\
\text { pact }^{33}\end{array}$ & $\begin{array}{l}\text { incident } \\
\text { analysis }\end{array}$ & impl & $\begin{array}{l}\text { base of } \\
\text { type } \\
\text { count }\end{array}$ & IMC:SG5.SP1 \\
\hline $\begin{array}{l}\text { IMC- } \\
\text { M20 }\end{array}$ & $\begin{array}{l}\text { number of incidents by incident type and root } \\
\text { cause }\end{array}$ & $\begin{array}{l}\text { incident } \\
\text { analysis }\end{array}$ & impl & $\begin{array}{l}\text { base of } \\
\text { type } \\
\text { count }\end{array}$ & IMC:SG5.SP1 \\
\hline $\begin{array}{l}\text { IMC- } \\
\text { M21 }\end{array}$ & impact due to incidents by incident type & $\begin{array}{l}\text { incident } \\
\text { analysis }\end{array}$ & impl & derived & IMC:SG5.SP1 \\
\hline $\begin{array}{l}\text { IMC- } \\
\text { M22 }\end{array}$ & $\begin{array}{l}\text { change in impact due to incidents by incident } \\
\text { type }\end{array}$ & $\begin{array}{l}\text { incident } \\
\text { analysis }\end{array}$ & $\begin{array}{l}\text { impl; possi- } \\
\text { bly effec- } \\
\text { tiveness }\end{array}$ & derived & IMC:SG5.SP1 \\
\hline $\begin{array}{l}\text { IMC- } \\
\text { M23 }\end{array}$ & percentage of incidents that recur & $\begin{array}{l}\text { incident } \\
\text { analysis }\end{array}$ & $\begin{array}{l}\text { impl; possi- } \\
\text { bly effec- } \\
\text { tiveness }\end{array}$ & derived & $\begin{array}{l}\text { IMC:SG3.SP2 } \\
\text { IMC:SG5.SP1 }\end{array}$ \\
\hline $\begin{array}{l}\text { IMC- } \\
\text { M24 }\end{array}$ & $\begin{array}{l}\text { percentage change in the number of incidents } \\
\text { by incident type }\end{array}$ & $\begin{array}{l}\text { incident } \\
\text { analysis }\end{array}$ & $\begin{array}{l}\text { impl; possi- } \\
\text { bly effec- } \\
\text { tiveness }\end{array}$ & derived & IMC:SG5.SP1 \\
\hline $\begin{array}{l}\text { IMC- } \\
\text { M25 }\end{array}$ & $\begin{array}{l}\text { time (mean, median, range) between event } \\
\text { detection and related incident declaration }\end{array}$ & $\begin{array}{l}\text { incident } \\
\text { analysis }\end{array}$ & $\begin{array}{l}\text { impl; possi- } \\
\text { bly effec- } \\
\text { tiveness }\end{array}$ & derived & IMC:SG5.SP1 \\
\hline $\begin{array}{l}\text { IMC- } \\
\text { M26 }\end{array}$ & $\begin{array}{l}\text { time (mean, median, range) between event } \\
\text { detection and related incident response }\end{array}$ & $\begin{array}{l}\text { incident } \\
\text { analysis }\end{array}$ & $\begin{array}{l}\text { impl; possi- } \\
\text { bly effec- } \\
\text { tiveness }\end{array}$ & derived & IMC:SG5.SP1 \\
\hline $\begin{array}{l}\text { IMC- } \\
\text { M27 }\end{array}$ & $\begin{array}{l}\text { time (mean, median, range) between event } \\
\text { detection and related incident closure }\end{array}$ & $\begin{array}{l}\text { incident } \\
\text { analysis }\end{array}$ & $\begin{array}{l}\text { impl; possi- } \\
\text { bly effec- } \\
\text { tiveness }\end{array}$ & derived & IMC:SG5.SP1 \\
\hline $\begin{array}{l}\text { IMC- } \\
\text { M28 }\end{array}$ & $\begin{array}{l}\text { percentage change in the elapsed time of the } \\
\text { incident life cycle by incident type (mean, me- } \\
\text { dian, ranges) }\end{array}$ & $\begin{array}{l}\text { incident } \\
\text { analysis }\end{array}$ & $\begin{array}{l}\text { impl; possi- } \\
\text { bly effec- } \\
\text { tiveness }\end{array}$ & derived & IMC:SG5.SP1 \\
\hline
\end{tabular}
33 Impact (i.e., the magnitude or consequences due to incidents) can be represented as monetary cost, productivi-
ty cost, loss of revenue due to unavailability of services, etc. 


\begin{tabular}{|c|c|c|c|c|c|}
\hline ID & Measure & $\begin{array}{l}\text { Type of } \\
\text { Information }\end{array}$ & $\begin{array}{l}\text { Measure } \\
\text { Type }\end{array}$ & $\begin{array}{l}\text { Base or } \\
\text { Derived }\end{array}$ & $\begin{array}{l}\text { Applicable } \\
\text { SG.SP }\end{array}$ \\
\hline $\begin{array}{l}\text { IMC- } \\
\text { M29 }\end{array}$ & $\begin{array}{l}\text { percentage of incidents that result in realized } \\
\text { risks that exceed established risk parameters }\end{array}$ & incident risk & $\begin{array}{l}\text { impl; possi- } \\
\text { bly effec- } \\
\text { tiveness }\end{array}$ & derived & none \\
\hline $\begin{array}{l}\text { IMC- } \\
\text { M30 }\end{array}$ & percentage of incidents that require escalation & $\begin{array}{l}\text { incident } \\
\text { escalation }\end{array}$ & impl & derived & IMC:SG4.SP1 \\
\hline $\begin{array}{l}\text { IMC- } \\
\text { M31 }\end{array}$ & $\begin{array}{l}\text { percentage of incidents that require involve- } \\
\text { ment of law enforcement }\end{array}$ & $\begin{array}{l}\text { incident } \\
\text { escalation }\end{array}$ & $\begin{array}{l}\text { impl; possi- } \\
\text { bly effec- } \\
\text { tiveness }\end{array}$ & derived & IMC:SG4.SP3 \\
\hline $\begin{array}{l}\text { IMC- } \\
\text { M32 }\end{array}$ & $\begin{array}{l}\text { percentage of incidents that require the in- } \\
\text { volvement of regulatory and governing agen- } \\
\text { cies }\end{array}$ & $\begin{array}{l}\text { incident } \\
\text { escalation }\end{array}$ & $\begin{array}{l}\text { impl; possi- } \\
\text { bly effec- } \\
\text { tiveness }\end{array}$ & derived & IMC:SG4.SP3 \\
\hline $\begin{array}{l}\text { IMC- } \\
\text { M33 }\end{array}$ & $\begin{array}{l}\text { percentage of post-incident review recommen- } \\
\text { dations that result in control changes or im- } \\
\text { provements to the process }\end{array}$ & $\begin{array}{l}\text { process } \\
\text { improvement }\end{array}$ & impl & derived & IMC:SG5.SP1 \\
\hline $\begin{array}{l}\text { IMC- } \\
\text { M34 }\end{array}$ & $\begin{array}{l}\text { number of problem reports referred to the prob- } \\
\text { lem management system }\end{array}$ & $\begin{array}{l}\text { process } \\
\text { improvement }\end{array}$ & impl & $\begin{array}{l}\text { base of } \\
\text { type } \\
\text { count }\end{array}$ & IMC:SG5.SP2 \\
\hline $\begin{array}{l}\text { IMC- } \\
\text { M35 }\end{array}$ & $\begin{array}{l}\text { extent to which incident occurrence (prevent) is } \\
\text { reduced as a result of implementing RMM ap- } \\
\text { praisal findings }\end{array}$ & $\begin{array}{l}\text { potential } \\
\text { element of } \\
\text { resilience } \\
\text { posture }\end{array}$ & $\begin{array}{l}\text { effective- } \\
\text { ness }\end{array}$ & derived & none \\
\hline $\begin{array}{l}\text { IMC- } \\
\text { M36 }\end{array}$ & $\begin{array}{l}\text { reduction in incident occurrence and impact } \\
\text { (detect, respond, recover) as a result of imple- } \\
\text { menting CERT-RMM appraisal findings }\end{array}$ & $\begin{array}{l}\text { potential } \\
\text { element of } \\
\text { resilience } \\
\text { posture }\end{array}$ & $\begin{array}{l}\text { effective- } \\
\text { ness }\end{array}$ & derived & none \\
\hline
\end{tabular}

\section{Knowledge and Information Management (KIM)}

The purpose of Knowledge and Information Management is to establish and manage an appropriate level of controls to support the confidentiality, integrity, and availability of the organization's information, vital records, and intellectual property.

\section{Summary of Specific Goals and Practices}

KIM:SG1 Establish and Prioritize Information Assets

KIM:SG1.SP1 Prioritize Information Assets

KIM:SG1.SP2 Categorize Information Assets

KIM:SG2 Protect Information Assets

KIM:SG2.SP1 Assign Resilience Requirements to Information Assets

KIM:SG2.SP2 Establish and Implement Controls

KIM:SG3 Manage Information Asset Risk

KIM:SG3.SP1 Identify and Assess Information Asset Risk

KIM:SG3.SP2 Mitigate Information Asset Risk

KIM:SG4 Manage Information Asset Confidentiality and Privacy

KIM:SG4.SP1 Encrypt High-Value Information

KIM:SG4.SP2 Control Access to Information Assets

KIM:SG4.SP3 Control Information Asset Disposition

34 Could include additional measures here for any of the roles listed in IMC:SG4.SP3 
KIM:SG5 Manage Information Asset Integrity

KIM:SG5.SP1 Control Modification of Information Assets

KIM:SG5.SP2 Manage Information Asset Configuration

KIM:SG5.SP3 Verify Validity of Information

KIM:SG6 Manage Information Asset Availability

KIM:SG6.SP1 Perform Information Duplication and Retention

KIM:SG6.SP2 Manage Organizational Knowledge

\section{Measures}

\begin{tabular}{|c|c|c|c|c|c|}
\hline ID & Measure & $\begin{array}{l}\text { Type of } \\
\text { Information }\end{array}$ & $\begin{array}{l}\text { Measure } \\
\text { Type }\end{array}$ & $\begin{array}{l}\text { Base or } \\
\text { Derived }\end{array}$ & $\begin{array}{l}\text { Applicable } \\
\text { SG.SP }\end{array}$ \\
\hline KIM-M1 & $\begin{array}{l}\text { percentage of information assets that } \\
\text { have been inventoried }\end{array}$ & $\begin{array}{l}\text { asset inven- } \\
\text { tory }\end{array}$ & impl & derived & $\begin{array}{l}\text { ADM:SG1.SP1 } \\
\text { KIM:SG1.SP1 }\end{array}$ \\
\hline KIM-M2 & $\begin{array}{l}\text { percentage of information assets } \\
\text { with/without a complete asset profile (such } \\
\text { as no stated resilience requirements) }\end{array}$ & $\begin{array}{l}\text { asset inven- } \\
\text { tory }\end{array}$ & impl & derived & $\begin{array}{l}\text { ADM:SG1.SP2 } \\
\text { KIM:SG2.SP1 }\end{array}$ \\
\hline KIM-M3 & $\begin{array}{l}\text { percentage of information assets } \\
\text { with/without a designated owner }\end{array}$ & $\begin{array}{l}\text { asset inven- } \\
\text { tory }\end{array}$ & impl & derived & ADM:SG1.SP3 \\
\hline KIM-M4 & $\begin{array}{l}\text { percentage of information assets } \\
\text { with/without a designated custodian (if } \\
\text { applicable) }\end{array}$ & $\begin{array}{l}\text { asset inven- } \\
\text { tory }\end{array}$ & impl & derived & ADM:SG1.SP3 \\
\hline KIM-M5 & $\begin{array}{l}\text { percentage of information assets that } \\
\text { have designated owners but no custo- } \\
\text { dians (if applicable) }\end{array}$ & $\begin{array}{l}\text { asset inven- } \\
\text { tory }\end{array}$ & impl & derived & ADM:SG1.SP3 \\
\hline KIM-M6 & $\begin{array}{l}\text { percentage of information assets that } \\
\text { have designated custodians but no own- } \\
\text { ers }\end{array}$ & $\begin{array}{l}\text { asset inven- } \\
\text { tory }\end{array}$ & impl & derived & ADM:SG1.SP3 \\
\hline KIM-M7 & $\begin{array}{l}\text { percentage of information assets that } \\
\text { have been inventoried, by service }\end{array}$ & $\begin{array}{l}\text { asset inven- } \\
\text { tory }\end{array}$ & impl & derived & ADM:SG2.SP1 \\
\hline KIM-M8 & $\begin{array}{l}\text { percentage of information assets that are } \\
\text { not associated with one or more services }\end{array}$ & $\begin{array}{l}\text { asset inven- } \\
\text { tory }\end{array}$ & impl & derived & ADM:SG2.SP1 \\
\hline KIM-M9 & $\begin{array}{l}\text { elapsed time since the information asset } \\
\text { inventory was reviewed }\end{array}$ & $\begin{array}{l}\text { asset inven- } \\
\text { tory }\end{array}$ & impl & $\begin{array}{l}\text { base of } \\
\text { type sche- } \\
\text { dule }\end{array}$ & $\begin{array}{l}\text { ADM:SG1.SP1 } \\
\text { ADM:SG3.SP1 }\end{array}$ \\
\hline KIM-M10 & $\begin{array}{l}\text { percentage of information asset-service } \\
\text { dependency conflicts with unimplemented } \\
\text { or incomplete mitigation plans }\end{array}$ & $\begin{array}{l}\text { asset-service } \\
\text { dependen- } \\
\text { cies }\end{array}$ & impl & derived & ADM:SG2.SP2 \\
\hline KIM-M11 & $\begin{array}{l}\text { percentage of information asset-service } \\
\text { dependency conflicts with no mitigation } \\
\text { plan }\end{array}$ & $\begin{array}{l}\text { asset-service } \\
\text { dependen- } \\
\text { cies }\end{array}$ & impl & derived & ADM:SG2.SP2 \\
\hline KIM-M12 & $\begin{array}{l}\text { number of discrepancies between the } \\
\text { current inventory and the previous inven- } \\
\text { tory }\end{array}$ & $\begin{array}{l}\text { asset inven- } \\
\text { tory }\end{array}$ & impl & $\begin{array}{l}\text { base of } \\
\text { type count }\end{array}$ & ADM:SG3.SP1 \\
\hline KIM-M13 & $\begin{array}{l}\text { number of changes made to asset profiles } \\
\text { in the information asset inventory }\end{array}$ & $\begin{array}{l}\text { asset inven- } \\
\text { tory }\end{array}$ & impl & $\begin{array}{l}\text { base of } \\
\text { type count }\end{array}$ & ADM:SG3.SP2 \\
\hline KIM-M14 & $\begin{array}{l}\text { number of changes to resilience require- } \\
\text { ments as a result of information asset } \\
\text { changes }\end{array}$ & $\begin{array}{l}\text { asset change } \\
\text { management }\end{array}$ & impl & $\begin{array}{l}\text { base of } \\
\text { type count }\end{array}$ & ADM:SG3.SP2 \\
\hline KIM-M15 & $\begin{array}{l}\text { number of changes to service continuity } \\
\text { plans as a result of information asset } \\
\text { changes }\end{array}$ & $\begin{array}{l}\text { asset change } \\
\text { management }\end{array}$ & impl & $\begin{array}{l}\text { base of } \\
\text { type count }\end{array}$ & ADM:SG3.SP2 \\
\hline KIM-M1 & $\begin{array}{l}\text { percentage of information assets that are } \\
\text { designated as high-value assets }\end{array}$ & $\begin{array}{l}\text { asset inven- } \\
\text { tory }\end{array}$ & impl & derived & KIM:SG1.SP1 \\
\hline
\end{tabular}




\begin{tabular}{|c|c|c|c|c|c|}
\hline ID & Measure & $\begin{array}{l}\text { Type of } \\
\text { Information }\end{array}$ & $\begin{array}{l}\text { Measure } \\
\text { Type }\end{array}$ & $\begin{array}{l}\text { Base or } \\
\text { Derived }\end{array}$ & $\begin{array}{l}\text { Applicable } \\
\text { SG.SP }\end{array}$ \\
\hline KIM-M16 & $\begin{array}{l}\text { elapsed time since review and validation } \\
\text { of high-value information assets and their } \\
\text { priorities }\end{array}$ & $\begin{array}{l}\text { asset inven- } \\
\text { tory }\end{array}$ & impl & derived & KIM:SG1.SP1 \\
\hline KIM-M17 & $\begin{array}{l}\text { number of information assets categorized } \\
\text { by service (includes number of assets that } \\
\text { support } 2 \text { or more, } 3 \text { or more, etc., servic- } \\
\text { es) }\end{array}$ & $\begin{array}{l}\text { asset inven- } \\
\text { tory }\end{array}$ & impl & $\begin{array}{l}\text { base of } \\
\text { type count }\end{array}$ & KIM:SG1.SP1 \\
\hline KIM-M18 & $\begin{array}{l}\text { percentage of information assets that } \\
\text { have not been categorized as to level of } \\
\text { sensitivity }\end{array}$ & $\begin{array}{l}\text { asset inven- } \\
\text { tory }\end{array}$ & impl & derived & KIM:SG1.SP2 \\
\hline KIM-M19 & $\begin{array}{l}\text { percentage of information assets without } \\
\text { assigned/defined resilience requirements }\end{array}$ & $\begin{array}{l}\text { asset re- } \\
\text { quirements }\end{array}$ & impl & derived & KIM:SG2.SP1 \\
\hline KIM-M20 & $\begin{array}{l}\text { percentage of information assets with } \\
\text { assigned/defined resilience requirements } \\
\text { that are undocumented }\end{array}$ & $\begin{array}{l}\text { asset re- } \\
\text { quirements }\end{array}$ & impl & derived & KIM:SG2.SP1 \\
\hline KIM-M21 & $\begin{array}{l}\text { percentage of information assets with no } \\
\text { (or missing) protection controls }\end{array}$ & $\begin{array}{l}\text { asset con- } \\
\text { trols }\end{array}$ & $\begin{array}{l}\text { impl; pos- } \\
\text { sibly effec- } \\
\text { tiveness }\end{array}$ & derived & KIM:SG2.SP2 \\
\hline KIM-M22 & $\begin{array}{l}\text { percentage of information assets with no } \\
\text { (or missing) sustainment controls }\end{array}$ & $\begin{array}{l}\text { asset con- } \\
\text { trols }\end{array}$ & $\begin{array}{l}\text { impl; pos- } \\
\text { sibly effec- } \\
\text { tiveness }\end{array}$ & derived & KIM:SG2.SP2 \\
\hline KIM-M23 & $\begin{array}{l}\text { percentage of information asset controls } \\
\text { (protection and sustainment) that are inef- } \\
\text { fective or inadequate as demonstrated by: } \\
\text { unsatisfied control objectives } \\
\text { unmet resilience requirements } \\
\text { outstanding control assessment problem } \\
\text { areas above established thresholds and } \\
\text { without remediation plans }\end{array}$ & $\begin{array}{l}\text { asset con- } \\
\text { trols }\end{array}$ & $\begin{array}{l}\text { impl; pos- } \\
\text { sibly effec- } \\
\text { tiveness }\end{array}$ & derived & KIM:SG2.SP2 \\
\hline KIM-M24 & $\begin{array}{l}\text { percentage of information asset control } \\
\text { deficiencies not resolved by scheduled } \\
\text { due date (refer to CTRL measures for } \\
\text { categories of control deficiencies ) }\end{array}$ & $\begin{array}{l}\text { asset con- } \\
\text { trols }\end{array}$ & impl & derived & KIM:SG2.SP2 \\
\hline KIM-M25 & $\begin{array}{l}\text { elapsed time since review of the effective- } \\
\text { ness of information asset controls }\end{array}$ & $\begin{array}{l}\text { asset con- } \\
\text { trols }\end{array}$ & impl & $\begin{array}{l}\text { base of } \\
\text { type sche- } \\
\text { dule }\end{array}$ & KIM:SG2.SP2 \\
\hline KIM-M26 & $\begin{array}{l}\text { elapsed time since risk assessment of } \\
\text { information assets performed }\end{array}$ & asset risk & impl & $\begin{array}{l}\text { base of } \\
\text { type sche- } \\
\text { dule }\end{array}$ & KIM:SG3.SP1 \\
\hline KIM-M27 & $\begin{array}{l}\text { elapsed time since business impact analy- } \\
\text { sis of information assets performed }\end{array}$ & asset risk & impl & $\begin{array}{l}\text { base of } \\
\text { type sche- } \\
\text { dule }\end{array}$ & KIM:SG3.SP1 \\
\hline KIM-M28 & $\begin{array}{l}\text { percentage of information assets for which } \\
\text { business impact valuation }{ }^{35} \text { has not been } \\
\text { performed }\end{array}$ & asset risk & impl & derived & KIM:SG3.SP1 \\
\hline KIM-M29 & $\begin{array}{l}\text { percentage of information assets for which } \\
\text { a risk assessment has not been performed } \\
\text { and documented (per policy or other } \\
\text { guideline) and according to plan }\end{array}$ & asset risk & impl & derived & KIM:SG3.SP1 \\
\hline KIM-M30 & $\begin{array}{l}\text { percentage of information asset risks that } \\
\text { have not been assigned to a responsible } \\
\text { party for action, tracking, and closure }\end{array}$ & asset risk & impl & derived & KIM:SG3.SP2 \\
\hline
\end{tabular}

35 Business impact valuation can be either qualitative (high, medium, low) or quantitative (based on levels of loss or damage, fines, number of customers lost, disruption in access, disclosure, alteration, destruction, etc.). 


\begin{tabular}{|c|c|c|c|c|c|}
\hline ID & Measure & $\begin{array}{l}\text { Type of } \\
\text { Information }\end{array}$ & $\begin{array}{l}\text { Measure } \\
\text { Type }\end{array}$ & $\begin{array}{l}\text { Base or } \\
\text { Derived }\end{array}$ & $\begin{array}{l}\text { Applicable } \\
\text { SG.SP }\end{array}$ \\
\hline KIM-M31 & $\begin{array}{l}\text { percentage of information asset risks } \\
\text { with a disposition of "mitigate or control" } \\
\text { that do not have a defined mitigation plan }\end{array}$ & asset risk & impl & derived & KIM:SG3.SP2 ${ }^{37}$ \\
\hline KIM-M32 & $\begin{array}{l}\text { percentage of information asset risks with } \\
\text { a "mitigate or control" disposition that are } \\
\text { not effectively mitigated by their mitigation } \\
\text { plans }\end{array}$ & asset risk & $\begin{array}{l}\text { effective- } \\
\text { ness }\end{array}$ & derived & KIM:SG3.SP2 \\
\hline KIM-M33 & $\begin{array}{l}\text { percentage of realized risks for information } \\
\text { assets that exceed established risk para- } \\
\text { meters }\end{array}$ & asset risk & $\begin{array}{l}\text { effective- } \\
\text { ness }\end{array}$ & derived & KIM:SG3.SP2 \\
\hline KIM-M34 & $\begin{array}{l}\text { number of violations of access control } \\
\text { policies for information assets } \\
\text { as a result, number of successful intru- } \\
\text { sions to technology assets (digital informa- } \\
\text { tion assets) or facility assets (physical } \\
\text { information assets) where information } \\
\text { assets "live" } \\
\text { as a result, number of information assets } \\
\text { that have been accessed in an unautho- } \\
\text { rized manner } \\
\text { as a result, number of incidents declared } \\
\text { as a result, number of breaches of confi- } \\
\text { dentiality and privacy }\end{array}$ & $\begin{array}{l}\text { asset intru- } \\
\text { sions; asset } \\
\text { integrity }\end{array}$ & $\begin{array}{l}\text { impl; pos- } \\
\text { sibly effec- } \\
\text { tiveness }\end{array}$ & $\begin{array}{l}\text { base of } \\
\text { type count }\end{array}$ & $\begin{array}{l}\text { KIM:SG4.SP2 } \\
\text { KIM:SG5.SP1 }\end{array}$ \\
\hline KIM-M35 & $\begin{array}{l}\text { percentage of information assets for which } \\
\text { encryption is required and not imple- } \\
\text { mented }\end{array}$ & $\begin{array}{l}\text { asset inven- } \\
\text { tory; asset } \\
\text { confidentiali- } \\
\text { ty }\end{array}$ & impl & derived & KIM:SG4.SP1 \\
\hline KIM-M36 & $\begin{array}{l}\text { percentage of retired information assets } \\
\text { that are not disposed of in accordance } \\
\text { with information asset disposition guide- } \\
\text { lines }\end{array}$ & $\begin{array}{l}\text { asset confi- } \\
\text { dentiality }\end{array}$ & impl & derived & KIM:SG4.SP3 \\
\hline KIM-M37 & $\begin{array}{l}\text { percentage of retired information assets } \\
\text { that have not been disposed according to } \\
\text { plan }\end{array}$ & $\begin{array}{l}\text { asset confi- } \\
\text { dentiality }\end{array}$ & impl & derived & KIM:SG4.SP3 \\
\hline KIM-M38 & $\begin{array}{l}\text { percentage of anomalies in information } \\
\text { asset modification logs that have not been } \\
\text { addressed as scheduled }\end{array}$ & $\begin{array}{l}\text { asset integri- } \\
\text { ty }\end{array}$ & impl & derived & KIM:SG5.SP1 \\
\hline KIM-M39 & $\begin{array}{l}\text { percentage of anomalies in information } \\
\text { asset configuration control logs that have } \\
\text { not been addressed as scheduled }\end{array}$ & $\begin{array}{l}\text { asset integri- } \\
\text { ty }\end{array}$ & impl & derived & KIM:SG5.SP2 \\
\hline KIM-M40 & $\begin{array}{l}\text { percentage of information asset logs } \\
\text { which are not validated and placed under } \\
\text { configuration control as scheduled }\end{array}$ & $\begin{array}{l}\text { asset integri- } \\
\text { ty }\end{array}$ & impl & derived & $\begin{array}{l}\text { KIM:SG5. SP1 } \\
\text { KIM:SG5.SP2 } \\
\text { KIM:SG5.SP3 }\end{array}$ \\
\hline KIM-M41 & $\begin{array}{l}\text { percentage of information assets with } \\
\text { accuracy and completeness controls that } \\
\text { have not been reviewed as scheduled }\end{array}$ & $\begin{array}{l}\text { asset integri- } \\
\text { ty }\end{array}$ & impl & derived & KIM:SG5.SP3 \\
\hline KIM-M42 & $\begin{array}{l}\text { percentage of information assets that } \\
\text { have not been backed up as scheduled }\end{array}$ & $\begin{array}{l}\text { asset availa- } \\
\text { bility }\end{array}$ & impl & derived & KIM:SG6.SP1 \\
\hline
\end{tabular}

36 This measure also appears in RISK M4-1. For ease of use of an individual PA (vs. ease of maintenance and consistency), we have decided to replicate some (but not all) risk-related measures in the individual asset PAs that are identified generally in the list of RISK PA measures.

37 SG3.SP2 subpractice 7 states, "Collect performance measures on the risk management process." No such measures are included here in KIM; refer to the RISK PA. 


\begin{tabular}{|c|c|c|c|c|c|}
\hline ID & Measure & $\begin{array}{l}\text { Type of } \\
\text { Information }\end{array}$ & $\begin{array}{l}\text { Measure } \\
\text { Type }\end{array}$ & $\begin{array}{l}\text { Base or } \\
\text { Derived }\end{array}$ & $\begin{array}{l}\text { Applicable } \\
\text { SG.SP }\end{array}$ \\
\hline KIM-M43 & $\begin{array}{l}\text { percentage of information assets that } \\
\text { have not been tested to verify that they } \\
\text { can be accurately restored from backups } \\
\text { as scheduled }\end{array}$ & $\begin{array}{l}\text { asset availa- } \\
\text { bility }\end{array}$ & impl & derived & KIM:SG6.SP1 \\
\hline KIM-M44 & $\begin{array}{l}\text { percentage of vital staff with institutional } \\
\text { knowledge where such knowledge has not } \\
\text { been captured/transferred (via such me- } \\
\text { thods as cross training) }\end{array}$ & $\begin{array}{l}\text { asset availa- } \\
\text { bility }\end{array}$ & impl & derived & KIM:SG6.SP2 \\
\hline KIM-M45 & $\begin{array}{l}\text { percentage of information assets that do } \\
\text { not satisfy their resilience requirements }\end{array}$ & $\begin{array}{l}\text { asset evalua- } \\
\text { tion }\end{array}$ & $\begin{array}{l}\text { impl; pos- } \\
\text { sibly effec- } \\
\text { tiveness }\end{array}$ & derived & $\begin{array}{l}\text { KIM:SG4, SG5, } \\
\text { SG6 }\end{array}$ \\
\hline KIM-M46 & $\begin{array}{l}\text { number of policy violations related to con- } \\
\text { fidentiality, integrity, availability, privacy, } \\
\text { and access control of information assets }\end{array}$ & $\begin{array}{l}\text { asset evalua- } \\
\text { tion }\end{array}$ & impl & $\begin{array}{l}\text { base of } \\
\text { type count }\end{array}$ & none \\
\hline KIM-M47 & $\begin{array}{l}\text { percentage of external entities that are not } \\
\text { meeting service level agreements for in- } \\
\text { formation assets subject to external entity } \\
\text { services }\end{array}$ & $\begin{array}{l}\text { asset evalua- } \\
\text { tion }\end{array}$ & impl & derived & none \\
\hline KIM-M48 & $\begin{array}{l}\text { percentage of information assets that are } \\
\text { not maintained at required maintenance } \\
\text { levels (for information assets subject to } \\
\text { maintenance agreements) }\end{array}$ & $\begin{array}{l}\text { asset evalua- } \\
\text { tion }\end{array}$ & impl & derived & none \\
\hline
\end{tabular}

\section{Measurement and Analysis (MA)}

The purpose of Measurement and Analysis is to develop and sustain a measurement capability that is used to support management information needs for managing the operational resilience management system.

\section{Summary of Specific Goals and Practices}

MA:SG1 Align Measurement and Analysis Activities

MA:SG1.SP1 Establish Measurement Objectives

\section{MA:SG1.SP2 Specify Measures}

MA:SG1.SP3 Specify Data Collection and Storage Procedures

MA:SG1.SP4 Specify Analysis Procedures

MA:SG2 Provide Measurement Results

MA:SG2.SP1 Collect Measurement Data

MA:SG2.SP2 Analyze Measurement Data

MA:SG2.SP3 Store Data and Results

MA:SG2.SP4 Communicate Results

Measures

\begin{tabular}{|l|l|l|l|l|l|}
\hline ID & Measure & $\begin{array}{l}\text { Type of } \\
\text { Information }\end{array}$ & $\begin{array}{l}\text { Measure } \\
\text { Type }\end{array}$ & $\begin{array}{l}\text { Base or } \\
\text { Derived }\end{array}$ & $\begin{array}{l}\text { Applicable } \\
\text { SG.SP }\end{array}$ \\
\hline MA-M1 & $\begin{array}{l}\text { percentage of measurement objectives that } \\
\text { can be traced to information needs and objec- } \\
\text { tives }\end{array}$ & $\begin{array}{l}\text { measurement } \\
\text { objectives }\end{array}$ & impl & derived & MA:SG1.SP1 \\
\hline MA-M2 & $\begin{array}{l}\text { percentage of measures for which operational } \\
\text { definitions have been specified }\end{array}$ & measures & impl & derived & MA:SG1.SP2 \\
\hline
\end{tabular}




\begin{tabular}{|l|l|l|l|l|l|}
\hline ID & Measure & $\begin{array}{l}\text { Type of } \\
\text { Information }\end{array}$ & $\begin{array}{l}\text { Measure } \\
\text { Type }\end{array}$ & $\begin{array}{l}\text { Base or } \\
\text { Derived }\end{array}$ & $\begin{array}{l}\text { Applicable } \\
\text { SG.SP }\end{array}$ \\
\hline MA-M3 & $\begin{array}{l}\text { percentage of measurement objectives } \\
\text { achieved (against defined targets, if relevant) }\end{array}$ & $\begin{array}{l}\text { measurement } \\
\text { objectives }\end{array}$ & $\begin{array}{l}\text { effective- } \\
\text { ness }\end{array}$ & derived & MA:SG2.SP2 \\
\hline MA-M4 & $\begin{array}{l}\text { percentage of operational resilience manage- } \\
\text { ment system performance goals for which } \\
\text { measurement data is collected, analyzed, and } \\
\text { communicated }\end{array}$ & $\begin{array}{l}\text { ORMS mea- } \\
\text { surement }\end{array}$ & impl & derived & $\begin{array}{l}\text { MA:SG2.SP1 } \\
\text { MA:SG2.SP2 } \\
\text { MA:SG2.SP4 }\end{array}$ \\
\hline MA-M5 & $\begin{array}{l}\text { percentage of organizational units, services, } \\
\text { and activities using operational resilience } \\
\text { management measures to assess the perfor- } \\
\text { mance of operational resilience management } \\
\text { processes }\end{array}$ & $\begin{array}{l}\text { ORMS mea- } \\
\text { surement }\end{array}$ & $\begin{array}{l}\text { impl; pos- } \\
\text { sibly effec- } \\
\text { tiveness }\end{array}$ & derived & MA:SG2.SP4 \\
\hline MA-M6 & $\begin{array}{l}\text { elapsed time between collection, analysis, and } \\
\text { communication of measurement data }\end{array}$ & $\begin{array}{l}\text { measurement } \\
\text { process }\end{array}$ & impl & $\begin{array}{l}\text { base of } \\
\text { type } \\
\text { schedule }\end{array}$ & $\begin{array}{l}\text { MA:SG2.SP1 } \\
\text { MA:SG2.SP2 } \\
\text { MA:SG2.SP4 }\end{array}$ \\
\hline MA-M7 & $\begin{array}{l}\text { percentage of measures that can be traced to } \\
\text { measurement objectives }\end{array}$ & $\begin{array}{l}\text { measurement } \\
\text { process }\end{array}$ & impl & derived & $\begin{array}{l}\text { MA:SG1.SP1 } \\
\text { MA.SG2.SP1 }\end{array}$ \\
\hline MA-M8 & $\begin{array}{l}\text { percentage of measures whose collection, } \\
\text { analysis, and reporting is automated }\end{array}$ & $\begin{array}{l}\text { measurement } \\
\text { process }\end{array}$ & impl & derived & $\begin{array}{l}\text { MA.SG2.SPI } \\
\text { MA.SG2.SP2 } \\
\text { MA.SG2.SP3 }\end{array}$ \\
\hline MA-M9 & $\begin{array}{l}\text { percentage of specified measures that are } \\
\text { collected, analyzed, and stored }\end{array}$ & measures & impl & derived & $\begin{array}{l}\text { MA:SG1.SP3 } \\
\text { MA:SG1.SP4 } \\
\text { MA:SG2.SP1 } \\
\text { MA:SG2.SP2 } \\
\text { MA:SG2.SP3 }\end{array}$ \\
\hline
\end{tabular}

\section{Monitoring (MON)}

The purpose of Monitoring is to collect, record, and distribute information about the operational resilience management system to the organization on a timely basis.

\section{Summary of Specific Goals and Practices}

MON:SG1 Establish and Maintain a Monitoring Program

MON:SG1.SP1 Establish a Monitoring Program

MON:SG1.SP2 Identify Stakeholders

MON:SG1.SP3 Establish Monitoring Requirements

MON:SG1.SP4 Analyze and Prioritize Monitoring Requirements

MON:SG2 Perform Monitoring

MON:SG2.SP1 Establish and Maintain Monitoring Infrastructure

MON:SG2.SP2 Establish Collection Standards and Guidelines

MON:SG2.SP3 Collect and Record Information

MON:SG2.SP4 Distribute Information

\section{Measures}

\begin{tabular}{|l|l|l|l|l|l|}
\hline ID & Measure & $\begin{array}{l}\text { Type of } \\
\text { Information }\end{array}$ & $\begin{array}{l}\text { Measure } \\
\text { Type }\end{array}$ & $\begin{array}{l}\text { Base or } \\
\text { Derived }\end{array}$ & $\begin{array}{l}\text { Applicable } \\
\text { SG.SP }\end{array}$ \\
\hline $\begin{array}{l}\text { MON- } \\
\text { M1 }\end{array}$ & $\begin{array}{l}\text { percentage of operational resilience man- } \\
\text { agement system performance goals for which } \\
\text { monitoring data is collected, recorded, and } \\
\text { distributed }\end{array}$ & $\begin{array}{l}\text { ORMS as- } \\
\text { sessment }\end{array}$ & impl & derived & $\begin{array}{l}\text { MON:SG2.SP3 } \\
\text { MON:SG2.SP4 }\end{array}$ \\
\hline
\end{tabular}




\begin{tabular}{|c|c|c|c|c|c|}
\hline ID & Measure & $\begin{array}{l}\text { Type of } \\
\text { Information }\end{array}$ & $\begin{array}{l}\text { Measure } \\
\text { Type }\end{array}$ & $\begin{array}{l}\text { Base or } \\
\text { Derived }\end{array}$ & $\begin{array}{l}\text { Applicable } \\
\text { SG.SP }\end{array}$ \\
\hline $\begin{array}{l}\text { MON- } \\
\text { M2 }\end{array}$ & $\begin{array}{l}\text { percentage of organizational units, services, } \\
\text { and activities using monitoring data to assess } \\
\text { the performance of operational resilience } \\
\text { management processes }\end{array}$ & $\begin{array}{l}\text { ORMS as- } \\
\text { sessment }\end{array}$ & $\begin{array}{l}\text { process } \\
\text { perfor- } \\
\text { mance }\end{array}$ & derived & none \\
\hline $\begin{array}{l}\text { MON- } \\
\text { M3 }\end{array}$ & $\begin{array}{l}\text { percentage of monitoring requirements ac- } \\
\text { cepted (accepted requirements divided by } \\
\text { total requirements) }\end{array}$ & $\begin{array}{l}\text { monitoring } \\
\text { coverage }\end{array}$ & impl & derived & $\begin{array}{l}\text { MON:SG1.SP3 } \\
\text { MON:SG1.SP4 }\end{array}$ \\
\hline $\begin{array}{l}\text { MON- } \\
\text { M4 }\end{array}$ & $\begin{array}{l}\text { number of requirements gaps (total require- } \\
\text { ments minus accepted requirements) }\end{array}$ & $\begin{array}{l}\text { monitoring } \\
\text { coverage }\end{array}$ & impl & $\begin{array}{l}\text { base of } \\
\text { type } \\
\text { count }\end{array}$ & $\begin{array}{l}\text { MON:SG1.SP3 } \\
\text { MON:SG1.SP4 }\end{array}$ \\
\hline $\begin{array}{l}\text { MON- } \\
\text { M5 }\end{array}$ & $\begin{array}{l}\text { number of ranked risks resulting from unsatis- } \\
\text { fied monitoring requirements }\end{array}$ & $\begin{array}{l}\text { risk identifi- } \\
\text { cation }\end{array}$ & impl & $\begin{array}{l}\text { base of } \\
\text { type } \\
\text { count }\end{array}$ & MON:SG1.SP4 \\
\hline $\begin{array}{l}\text { MON- } \\
\text { M6 }\end{array}$ & $\begin{array}{l}\text { elapsed time from high-value data collection } \\
\text { to data distribution to key stakeholders }\end{array}$ & $\begin{array}{l}\text { monitoring } \\
\text { communica- } \\
\text { tion }\end{array}$ & $\begin{array}{l}\text { effective- } \\
\text { ness }\end{array}$ & $\begin{array}{l}\text { base of } \\
\text { type } \\
\text { schedule }\end{array}$ & MON:SG2.SP4 \\
\hline $\begin{array}{l}\text { MON- } \\
\text { M7 }\end{array}$ & $\begin{array}{l}\text { number of new, changed, and retired monitor- } \\
\text { ing requirements }\end{array}$ & $\begin{array}{l}\text { monitoring } \\
\text { variability }\end{array}$ & impl & $\begin{array}{l}\text { base of } \\
\text { type } \\
\text { count }\end{array}$ & MON:SG1.SP3 \\
\hline $\begin{array}{l}\text { MON- } \\
\text { M8 }\end{array}$ & $\begin{array}{l}\text { number of times monitoring plan has been } \\
\text { revised }\end{array}$ & $\begin{array}{l}\text { monitoring } \\
\text { variability }\end{array}$ & impl & $\begin{array}{l}\text { base of } \\
\text { type } \\
\text { count }\end{array}$ & MON:SG1.SP1 \\
\hline $\begin{array}{l}\text { MON- } \\
\text { M9 }\end{array}$ & $\begin{array}{l}\text { percentage of data collection activities that } \\
\text { are automated }\end{array}$ & $\begin{array}{l}\text { monitoring } \\
\text { process }\end{array}$ & impl & derived & MON:SG2.SP3 \\
\hline
\end{tabular}

\section{Organization Process Definition (OPD)}

The purpose of Organizational Process Definition is to establish and maintain a usable set of organizational process assets and work environment standards for operational resilience.

\section{Summary of Specific Goals and Practices}

OPD:SG1 Establish Organizational Process Assets

OPD:SG1.SP1 Establish Standard Processes

OPD:SG1.SP2 Establish Tailoring Criteria and Guidelines

OPD:SG1.SP3 Establish the Organization's Measurement Repository

OPD:SG1.SP4 Establish the Organization's Process Asset Library

OPD:SG1.SP5 Establish Work Environment Standards

OPD:SG1.SP6 Establish Rules and Guidelines for Integrated Teams

\section{Measures}

\begin{tabular}{|l|l|l|l|l|l|}
\hline ID & Measure & $\begin{array}{l}\text { Type of } \\
\text { Information }\end{array}$ & $\begin{array}{l}\text { Measure } \\
\text { Type }\end{array}$ & $\begin{array}{l}\text { Base or } \\
\text { Derived }\end{array}$ & $\begin{array}{l}\text { Applicable } \\
\text { SG.SP }\end{array}$ \\
\hline $\begin{array}{l}\text { OPD- } \\
\text { M1 }\end{array}$ & $\begin{array}{l}\text { percentage of organizational units (including } \\
\text { projects) using the organization's standard } \\
\text { processes }\end{array}$ & $\begin{array}{l}\text { standard } \\
\text { process } \\
\text { deployment }\end{array}$ & impl & derived & OPD:SG1.SP1 \\
\hline $\begin{array}{l}\text { OPD- } \\
\text { M2 }\end{array}$ & $\begin{array}{l}\text { percentage of standard processes that map to } \\
\text { process policies, standards, or models }\end{array}$ & $\begin{array}{l}\text { standard } \\
\text { process } \\
\text { develop- } \\
\text { ment }\end{array}$ & impl & derived & OPD:SG1.SP1 \\
\hline
\end{tabular}




\begin{tabular}{|c|c|c|c|c|c|}
\hline ID & Measure & $\begin{array}{l}\text { Type of } \\
\text { Information }\end{array}$ & $\begin{array}{l}\text { Measure } \\
\text { Type }\end{array}$ & $\begin{array}{l}\text { Base or } \\
\text { Derived }\end{array}$ & $\begin{array}{l}\text { Applicable } \\
\text { SG.SP }\end{array}$ \\
\hline $\begin{array}{l}\text { OPD- } \\
\text { M3 }\end{array}$ & $\begin{array}{l}\text { percentage of standard processes that satisfy } \\
\text { process needs and objectives }\end{array}$ & $\begin{array}{l}\text { standard } \\
\text { process } \\
\text { develop- } \\
\text { ment }\end{array}$ & impl & derived & OPD:SG1.SP1 \\
\hline $\begin{array}{l}\text { OPD- } \\
\text { M4 }\end{array}$ & $\begin{array}{l}\text { percentage of standard processes that have } \\
\text { been peer reviewed }\end{array}$ & $\begin{array}{l}\text { standard } \\
\text { process } \\
\text { develop- } \\
\text { ment }\end{array}$ & impl & derived & OPD:SG1.SP1 \\
\hline $\begin{array}{l}\text { OPD- } \\
\text { M5 }\end{array}$ & $\begin{array}{l}\text { percentage of standard processes that have } \\
\text { been tailored, by organizational unit }\end{array}$ & $\begin{array}{l}\text { standard } \\
\text { process use }\end{array}$ & impl & derived & OPD:SG1.SP2 \\
\hline $\begin{array}{l}\text { OPD- } \\
\text { M6 }\end{array}$ & $\begin{array}{l}\text { number of times a standard process has been } \\
\text { tailored }\end{array}$ & $\begin{array}{l}\text { standard } \\
\text { process use }\end{array}$ & impl & $\begin{array}{l}\text { base of } \\
\text { type } \\
\text { count }\end{array}$ & OPD:SG1.SP2 \\
\hline $\begin{array}{l}\text { OPD- } \\
\text { M7 }\end{array}$ & number of waivers by standard process & $\begin{array}{l}\text { standard } \\
\text { process } \\
\text { deployment }\end{array}$ & impl & $\begin{array}{l}\text { base of } \\
\text { type } \\
\text { count }\end{array}$ & OPD:SG1.SP2 \\
\hline $\begin{array}{l}\text { OPD- } \\
\text { M8 }\end{array}$ & $\begin{array}{l}\text { percentage of tailoring guidelines that have } \\
\text { been peer reviewed }\end{array}$ & $\begin{array}{l}\text { tailoring } \\
\text { guideline } \\
\text { develop- } \\
\text { ment }\end{array}$ & impl & derived & OPD:SG1.SP2 \\
\hline $\begin{array}{l}\text { OPD- } \\
\text { M9 }\end{array}$ & $\begin{array}{l}\text { defect density of each process element of the } \\
\text { organization's set of standard processes }\end{array}$ & $\begin{array}{l}\text { standard } \\
\text { process } \\
\text { develop- } \\
\text { ment }\end{array}$ & $\begin{array}{l}\text { effective- } \\
\text { ness }\end{array}$ & derived & OPD:SG1.SP1 \\
\hline $\begin{array}{l}\text { OPD- } \\
\text { M10 }\end{array}$ & $\begin{array}{l}\text { elapsed time for development of a standard } \\
\text { process (mean, median) }\end{array}$ & $\begin{array}{l}\text { standard } \\
\text { process } \\
\text { develop- } \\
\text { ment } \\
\end{array}$ & impl & $\begin{array}{l}\text { base of } \\
\text { type } \\
\text { schedule }\end{array}$ & OPD:SG1.SP1 \\
\hline $\begin{array}{l}\text { OPD- } \\
\text { M11 }\end{array}$ & $\begin{array}{l}\text { elapsed time for changes to a standard } \\
\text { process (mean, median) }\end{array}$ & $\begin{array}{l}\text { standard } \\
\text { process } \\
\text { mainten- } \\
\text { ance }\end{array}$ & impl & $\begin{array}{l}\text { base of } \\
\text { type } \\
\text { schedule }\end{array}$ & OPD:SG1.SP1 \\
\hline $\begin{array}{l}\text { OPD- } \\
\text { M12 }\end{array}$ & $\begin{array}{l}\text { number of unapproved changes to the } \\
\text { process asset library }\end{array}$ & $\begin{array}{l}\text { process } \\
\text { asset main- } \\
\text { tenance }\end{array}$ & impl & $\begin{array}{l}\text { base of } \\
\text { type } \\
\text { count }\end{array}$ & OPD:SG1.SP4 \\
\hline $\begin{array}{l}\text { OPD- } \\
\text { M13 }\end{array}$ & $\begin{array}{l}\text { number of times each item in the process } \\
\text { assets library is accessed }\end{array}$ & $\begin{array}{l}\text { process } \\
\text { asset library } \\
\text { use }\end{array}$ & impl & $\begin{array}{l}\text { base of } \\
\text { type } \\
\text { count }\end{array}$ & OPD:SG1.SP4 \\
\hline $\begin{array}{l}\text { OPD- } \\
\text { M14 }\end{array}$ & $\begin{array}{l}\text { percentage of product and process measures } \\
\text { residing in the measurement repository that } \\
\text { are used in status reports }\end{array}$ & $\begin{array}{l}\text { measure- } \\
\text { ment reposi- } \\
\text { tory }\end{array}$ & impl & derived & OPD:SG1.SP3 \\
\hline $\begin{array}{l}\text { OPD- } \\
\text { M15 }\end{array}$ & $\begin{array}{l}\text { number of waivers by work environment stan- } \\
\text { dard }\end{array}$ & $\begin{array}{l}\text { work envi- } \\
\text { ronment } \\
\text { standards }\end{array}$ & impl & $\begin{array}{l}\text { base of } \\
\text { type } \\
\text { count }\end{array}$ & OPD:SG1.SP5 \\
\hline $\begin{array}{l}\text { OPD- } \\
\text { M16 }\end{array}$ & $\begin{array}{l}\text { number of worker's compensation claims due } \\
\text { to work environment }\end{array}$ & $\begin{array}{l}\text { work envi- } \\
\text { ronment } \\
\text { standards }\end{array}$ & impl & $\begin{array}{l}\text { base of } \\
\text { type } \\
\text { count }\end{array}$ & OPD:SG1.SP5 \\
\hline
\end{tabular}

\section{Organizational Process Focus (OPF)}

The purpose of Organizational Process Focus is to plan, implement, and deploy organizational process improvements based on a thorough understanding of current strengths and weaknesses of the organization's operational resilience processes and process assets. 


\section{Summary of Specific Goals and Practices}

OPF:SG1 Determine Process Improvement Opportunities

OPF:SG1.SP1 Establish Organizational Process Needs

OPF:SG1.SP2 Appraise the Organization's Processes

OPF:SG1.SP3 Identify the Organization's Process Improvements

OPF:SG2 Plan and Implement Process Actions

OPF:SG2.SP1 Establish Process Action Plans

OPF:SG2.SP2 Implement Process Action Plans

OPF:SG3 Deploy Organizational Process Assets and Incorporate Experiences

OPF:SG3.SP1 Deploy Organizational Process Assets

OPF:SG3.SP2 Deploy Standard Processes

OPF:SG3.SP3 Monitor the Implementation

OPF:SG3.SP4 Incorporate Experiences into Organizational Process Assets

\section{Measures}

\begin{tabular}{|c|c|c|c|c|c|}
\hline ID & Measure & $\begin{array}{l}\text { Type of } \\
\text { Information }\end{array}$ & $\begin{array}{l}\text { Measure } \\
\text { Type }\end{array}$ & $\begin{array}{l}\text { Base or } \\
\text { Derived }\end{array}$ & $\begin{array}{l}\text { Applicable } \\
\text { SG.SP }\end{array}$ \\
\hline $\begin{array}{l}\text { OPF- } \\
\text { M1 }\end{array}$ & $\begin{array}{l}\text { percentage of process improvement propos- } \\
\text { als accepted }\end{array}$ & $\begin{array}{l}\text { process } \\
\text { improve- } \\
\text { ment }\end{array}$ & impl & derived & OPF:SG1.SP3 \\
\hline $\begin{array}{l}\text { OPF- } \\
\text { M2 }\end{array}$ & $\begin{array}{l}\text { percentage of planned process improvements } \\
\text { implemented }\end{array}$ & $\begin{array}{l}\text { process } \\
\text { improve- } \\
\text { ment }\end{array}$ & impl & derived & OPF:SG2.SP2 \\
\hline $\begin{array}{l}\text { OPF- } \\
\text { M3 }\end{array}$ & $\begin{array}{l}\text { percentage of improvements resulting from } \\
\text { appraisals }\end{array}$ & $\begin{array}{l}\text { process } \\
\text { improve- } \\
\text { ment }\end{array}$ & impl & derived & $\begin{array}{l}\text { OPF:SG1.SP2 } \\
\text { OPF:SG1.SP3 }\end{array}$ \\
\hline $\begin{array}{l}\text { OPF- } \\
\text { M4 }\end{array}$ & $\begin{array}{l}\text { percentage of improvements resulting from } \\
\text { experience reports and lessons learned }\end{array}$ & $\begin{array}{l}\text { process } \\
\text { improve- } \\
\text { ment }\end{array}$ & impl & derived & $\begin{array}{l}\text { OPF:SG1.SP3 } \\
\text { OPF:SG3.SP4 }\end{array}$ \\
\hline $\begin{array}{l}\text { OPF- } \\
\text { M5 }\end{array}$ & $\begin{array}{l}\text { CERT Resilience Management Model capa- } \\
\text { bility levels }\end{array}$ & $\begin{array}{l}\text { process } \\
\text { capability }\end{array}$ & $\begin{array}{l}\text { effective- } \\
\text { ness }\end{array}$ & derived & OPF:SG1.SP2 \\
\hline $\begin{array}{l}\text { OPF- } \\
\text { M6 }\end{array}$ & $\begin{array}{l}\text { elapsed time for deployment of an organiza- } \\
\text { tional process asset }\end{array}$ & $\begin{array}{l}\text { process } \\
\text { asset dep- } \\
\text { loyment }\end{array}$ & impl & $\begin{array}{l}\text { base of } \\
\text { type } \\
\text { schedule }\end{array}$ & OPF:SG3.SP1 \\
\hline $\begin{array}{l}\text { OPF- } \\
\text { M7 }\end{array}$ & $\begin{array}{l}\text { status against schedule for deployment of an } \\
\text { organizational process asset (i.e., met or } \\
\text { exceeded and by how much) }\end{array}$ & $\begin{array}{l}\text { process } \\
\text { asset dep- } \\
\text { loyment }\end{array}$ & $\begin{array}{l}\text { effective- } \\
\text { ness }\end{array}$ & derived & OPF:SG3.SP1 \\
\hline $\begin{array}{l}\text { OPF- } \\
\text { M8 }\end{array}$ & $\begin{array}{l}\text { percentage of organizational units using the } \\
\text { organization's current set of standard } \\
\text { processes (or tailored versions of same) }\end{array}$ & $\begin{array}{l}\text { standard } \\
\text { process } \\
\text { deployment }\end{array}$ & impl & derived & OPF:SG3.SP2 \\
\hline $\begin{array}{l}\text { OPF- } \\
\text { M9 }\end{array}$ & $\begin{array}{l}\text { issue trends associated with implementing the } \\
\text { organization's set of standard processes (i.e., } \\
\text { number of issues identified and number } \\
\text { closed) }\end{array}$ & $\begin{array}{l}\text { standard } \\
\text { process } \\
\text { deployment }\end{array}$ & $\begin{array}{l}\text { effective- } \\
\text { ness }\end{array}$ & derived & OPF:SG3.SP3 \\
\hline $\begin{array}{l}\text { OPF- } \\
\text { M10 }\end{array}$ & $\begin{array}{l}\text { percentage of waivers approved/rejected by } \\
\text { standard process }\end{array}$ & $\begin{array}{l}\text { standard } \\
\text { process } \\
\text { deployment }\end{array}$ & impl & derived & OPF:SG3.SP4 \\
\hline $\begin{array}{l}\text { OPF- } \\
\text { M11 }\end{array}$ & $\begin{array}{l}\text { percentage of standard processes that have } \\
\text { been tailored, by organizational unit }\end{array}$ & $\begin{array}{l}\text { standard } \\
\text { process } \\
\text { tailoring }\end{array}$ & impl & derived & OPF:SG3.SP2 \\
\hline
\end{tabular}




\begin{tabular}{|c|c|c|c|c|c|}
\hline ID & Measure & $\begin{array}{l}\text { Type of } \\
\text { Information }\end{array}$ & $\begin{array}{l}\text { Measure } \\
\text { Type }\end{array}$ & $\begin{array}{l}\text { Base or } \\
\text { Derived }\end{array}$ & $\begin{array}{l}\text { Applicable } \\
\text { SG.SP }\end{array}$ \\
\hline $\begin{array}{l}\text { OPF- } \\
\text { M12 }\end{array}$ & $\begin{array}{l}\text { number of times a standard process has been } \\
\text { tailored }\end{array}$ & $\begin{array}{l}\text { standard } \\
\text { process use }\end{array}$ & impl & $\begin{array}{l}\text { base of } \\
\text { type } \\
\text { count }\end{array}$ & OPF:SG3.SP4 \\
\hline $\begin{array}{l}\text { OPF- } \\
\text { M13 }\end{array}$ & $\begin{array}{l}\text { progress toward achievement of process } \\
\text { needs and objectives }\end{array}$ & $\begin{array}{l}\text { process } \\
\text { objectives }\end{array}$ & $\begin{array}{l}\text { effective- } \\
\text { ness }\end{array}$ & derived & OPF:SG1.SP1 \\
\hline $\begin{array}{l}\text { OPF- } \\
\text { M14 }\end{array}$ & $\begin{array}{l}\text { percentage of processes that can be mapped } \\
\text { directly to documented critical success factors } \\
\text { or an enterprise strategy }\end{array}$ & $\begin{array}{l}\text { process } \\
\text { objectives }\end{array}$ & impl & derived & OPF:SG1.SP1 \\
\hline
\end{tabular}

\section{Organization Training and Awareness (OTA)}

The purpose of Organizational Training and Awareness is to promote awareness in and develop skills and knowledge of people in support of their roles in attaining and sustaining operational resilience.

\section{Summary of Specific Goals and Practices}

OTA:SG1 Establish Awareness Program

OTA:SG1.SP1 Establish Awareness Needs

OTA:SG1.SP2 Establish Awareness Plan

OTA:SG1.SP3 Establish Awareness Delivery Capability

OTA:SG2 Conduct Awareness Activities

OTA:SG2.SP1 Perform Awareness Activities

OTA:SG2.SP2 Establish Awareness Records

OTA:SG2.SP3 Assess Awareness Program Effectiveness

OTA:SG3 Establish Training Capability

OTA:SG3.SP1 Establish Training Needs

OTA:SG3.SP2 Establish Training Plan

OTA:SG3.SP3 Establish Training Capability

OTA:SG4 Conduct Training

OTA:SG4.SP1 Deliver Training

OTA:SG4.SP2 Establish Training Records

OTA:SG4.SP3 Assess Training Effectiveness

\section{Measures}

\begin{tabular}{|l|l|l|l|l|l|}
\hline ID & Measure & $\begin{array}{l}\text { Type of } \\
\text { Information }\end{array}$ & $\begin{array}{l}\text { Measure } \\
\text { Type }\end{array}$ & $\begin{array}{l}\text { Base or } \\
\text { Derived }\end{array}$ & $\begin{array}{l}\text { Applicable } \\
\text { SG.SP }\end{array}$ \\
\hline $\begin{array}{l}\text { OTA- } \\
\text { M1 }\end{array}$ & $\begin{array}{l}\text { percentage of awareness needs for each } \\
\text { staff group that are addressed in the } \\
\text { awareness plan }\end{array}$ & $\begin{array}{l}\text { awareness } \\
\text { needs; aware- } \\
\text { ness activities }\end{array}$ & impl & derived & $\begin{array}{l}\text { OTA:SG1.SP1 } \\
\text { OTA:SG1.SP2 }\end{array}$ \\
\hline $\begin{array}{l}\text { OTA- } \\
\text { M2 }\end{array}$ & $\begin{array}{l}\text { difference in planned versus actual } \\
\text { awareness sessions delivered }\end{array}$ & $\begin{array}{l}\text { awareness } \\
\text { activities }\end{array}$ & impl & derived & $\begin{array}{l}\text { OTA:SG1.SP2 } \\
\text { OTA:SG2.SP1 }\end{array}$ \\
\hline $\begin{array}{l}\text { OTA- } \\
\text { M3 }\end{array}$ & $\begin{array}{l}\text { schedule of delivery of awareness ses- } \\
\text { sions (planned frequency versus actual } \\
\text { frequency) }\end{array}$ & $\begin{array}{l}\text { awareness } \\
\text { activities }\end{array}$ & impl & derived & $\begin{array}{l}\text { OTA:SG1.SP2 } \\
\text { OTA:SG2.SP1 }\end{array}$ \\
\hline
\end{tabular}




\begin{tabular}{|c|c|c|c|c|c|}
\hline ID & Measure & $\begin{array}{l}\text { Type of } \\
\text { Information }\end{array}$ & $\begin{array}{l}\text { Measure } \\
\text { Type }\end{array}$ & $\begin{array}{l}\text { Base or } \\
\text { Derived }\end{array}$ & $\begin{array}{l}\text { Applicable } \\
\text { SG.SP }\end{array}$ \\
\hline $\begin{array}{l}\text { OTA- } \\
\text { M4 }\end{array}$ & $\begin{array}{l}\text { elapsed time since awareness materials } \\
\text { were reviewed and updated }\end{array}$ & $\begin{array}{l}\text { awareness } \\
\text { activities }\end{array}$ & impl & $\begin{array}{l}\text { base of } \\
\text { type sche- } \\
\text { dule }\end{array}$ & OTA:SG1.SP3 \\
\hline $\begin{array}{l}\text { OTA- } \\
\text { M5 }\end{array}$ & $\begin{array}{l}\text { percentage of new users (internal and } \\
\text { external) who have satisfactorily com- } \\
\text { pleted awareness sessions before being } \\
\text { granted network access }\end{array}$ & $\begin{array}{l}\text { awareness } \\
\text { activities; } \\
\text { awareness } \\
\text { requirements }\end{array}$ & impl & derived & OTA:SG2.SP2 \\
\hline $\begin{array}{l}\text { OTA- } \\
\text { M6 }\end{array}$ & $\begin{array}{l}\text { percentage of users (internal and exter- } \\
\text { nal) who have satisfactorily completed } \\
\text { periodic awareness refresher sessions as } \\
\text { required by policy }\end{array}$ & $\begin{array}{l}\text { awareness } \\
\text { activities; } \\
\text { awareness } \\
\text { requirements }\end{array}$ & impl & derived & OTA:SG2.SP2 \\
\hline $\begin{array}{l}\text { OTA- } \\
\text { M7 }\end{array}$ & $\begin{array}{l}\text { percentage of awareness activities that } \\
\text { include a mechanism for evaluating the } \\
\text { effectiveness of the awareness activity }\end{array}$ & $\begin{array}{l}\text { awareness } \\
\text { activities }\end{array}$ & impl & derived & OTA:SG2.SP3 \\
\hline $\begin{array}{l}\text { OTA- } \\
\text { M8 }\end{array}$ & $\begin{array}{l}\text { percentage of passing scores (by partici- } \\
\text { pants) on awareness assessments }\end{array}$ & $\begin{array}{l}\text { awareness } \\
\text { assessments }\end{array}$ & effectiveness & derived & OTA:SG2.SP3 \\
\hline $\begin{array}{l}\text { OTA- } \\
\text { M9 }\end{array}$ & $\begin{array}{l}\text { percentage of staff who have been as- } \\
\text { sessed to determine if their level of } \\
\text { awareness is commensurate with their } \\
\text { job responsibilities }\end{array}$ & $\begin{array}{l}\text { awareness } \\
\text { assessments }\end{array}$ & effectiveness & derived & OTA:SG2.SP3 \\
\hline $\begin{array}{l}\text { OTA- } \\
\text { M10 }\end{array}$ & $\begin{array}{l}\text { percentage of staff waived from aware- } \\
\text { ness activities }\end{array}$ & $\begin{array}{l}\text { awareness } \\
\text { waivers }\end{array}$ & impl & derived & OTA:SG2.SP2 \\
\hline $\begin{array}{l}\text { OTA- } \\
\text { M11 }\end{array}$ & $\begin{array}{l}\text { percentage of training needs for each } \\
\text { role and responsibility that are addressed } \\
\text { in the training plan }\end{array}$ & $\begin{array}{l}\text { training needs; } \\
\text { training courses }\end{array}$ & impl & derived & $\begin{array}{l}\text { OTA:SG3.SP1 } \\
\text { OTA:SG3.SP2 }\end{array}$ \\
\hline $\begin{array}{l}\text { OTA- } \\
\text { M12 }\end{array}$ & $\begin{array}{l}\text { difference in planned versus actual train- } \\
\text { ing courses delivered }\end{array}$ & training courses & impl & derived & $\begin{array}{l}\text { OTA:SG3.SP2 } \\
\text { OTA:SG4.SP1 }\end{array}$ \\
\hline $\begin{array}{l}\text { OTA- } \\
\text { M13 }\end{array}$ & $\begin{array}{l}\text { schedule of delivery of training sessions } \\
\text { (planned frequency versus actual fre- } \\
\text { quency) }\end{array}$ & training courses & impl & derived & $\begin{array}{l}\text { OTA:SG3.SP2 } \\
\text { OTA:SG4.SP1 }\end{array}$ \\
\hline $\begin{array}{l}\text { OTA- } \\
\text { M14 }\end{array}$ & $\begin{array}{l}\text { percentage of favorable post-training } \\
\text { evaluation ratings, including instructor } \\
\text { ratings }\end{array}$ & training courses & effectiveness & derived & OTA:SG4.SP3 \\
\hline $\begin{array}{l}\text { OTA- } \\
\text { M15 }\end{array}$ & $\begin{array}{l}\text { elapsed time since training materials } \\
\text { were reviewed and updated }\end{array}$ & $\begin{array}{l}\text { training mate- } \\
\text { rials }\end{array}$ & impl & $\begin{array}{l}\text { base of } \\
\text { type sche- } \\
\text { dule }\end{array}$ & OTA:SG3.SP3 \\
\hline $\begin{array}{l}\text { OTA- } \\
\text { M16 }\end{array}$ & $\begin{array}{l}\text { number of internal staff members for } \\
\text { whom training was planned versus num- } \\
\text { ber trained (percentage) }\end{array}$ & staff training & impl & derived & OTA:SG4.SP1 \\
\hline $\begin{array}{l}\text { OTA- } \\
\text { M17 }\end{array}$ & $\begin{array}{l}\text { number of external staff members for } \\
\text { whom training was expected or con- } \\
\text { tracted versus number trained (percen- } \\
\text { tage) }\end{array}$ & staff training & impl & derived & OTA:SG4.SP1 \\
\hline $\begin{array}{l}\text { OTA- } \\
\text { M18 }\end{array}$ & $\begin{array}{l}\text { percentage of favorable training program } \\
\text { quality survey ratings }\end{array}$ & $\begin{array}{l}\text { training pro- } \\
\text { gram }\end{array}$ & effectiveness & derived & OTA:SG4.SP3 \\
\hline $\begin{array}{l}\text { OTA- } \\
\text { M19 }\end{array}$ & $\begin{array}{l}\text { percentage of passing scores (by partici- } \\
\text { pants) on training examinations }\end{array}$ & $\begin{array}{l}\text { training exami- } \\
\text { nations }\end{array}$ & effectiveness & derived & OTA:SG4.SP2 \\
\hline $\begin{array}{l}\text { OTA- } \\
\text { M20 }\end{array}$ & $\begin{array}{l}\text { percentage of staff who have been as- } \\
\text { sessed to determine if training has been } \\
\text { effective }{ }^{38} \text { commensurate with their job } \\
\text { responsibilities }\end{array}$ & $\begin{array}{l}\text { training as- } \\
\text { sessment }\end{array}$ & effectiveness & derived & OTA:SG4.SP3 \\
\hline $\begin{array}{l}\text { OTA- } \\
\text { M21 }\end{array}$ & percentage of staff waived from training & training waivers & impl & derived & OTA:SG4.SP2 \\
\hline
\end{tabular}

38 OTA:SG4.SP3 provides several approaches for assessing training effectiveness. 


\section{People Management (PM)}

The purpose of People Management is to establish and manage the contributions and availability of people to support the resilient operation of organizational services.

\section{Summary of Specific Goals and Practices}

PM:SG1 Establish Vital Staff

PM:SG1.SP1 Identify Vital Staff

PM:SG2 Manage Risks Associated with Staff Availability

PM:SG2.SP1 Identify and Assess Staff Risk

PM:SG2.SP2 Mitigate Staff Risk

PM:SG3 Manage the Availability of Staff

PM:SG3.SP1 Establish Redundancy for Vital Staff

PM:SG3.SP2 Perform Succession Planning

PM:SG3.SP3 Prepare for Redeployment

PM:SG3.SP4 Plan to Support Staff During Disruptive Events

PM:SG3.SP5 Plan for Return-to-Work Considerations

\section{Measures}

\begin{tabular}{|c|c|c|c|c|c|}
\hline ID & Measure & $\begin{array}{l}\text { Type of } \\
\text { Information }\end{array}$ & $\begin{array}{l}\text { Measure } \\
\text { Type }\end{array}$ & $\begin{array}{l}\text { Base or } \\
\text { Derived }\end{array}$ & $\begin{array}{l}\text { Applicable } \\
\text { SG.SP }\end{array}$ \\
\hline PM-M1 & $\begin{array}{l}\text { percentage of staff-service dependency } \\
\text { conflicts with unimplemented or incom- } \\
\text { plete mitigation plans }\end{array}$ & $\begin{array}{l}\text { asset-service } \\
\text { dependencies; } \\
\text { risk mitigation }\end{array}$ & impl & derived & $\begin{array}{l}\text { ADM:SG2.SP2 } \\
\text { PM:SG2.SP1 } \\
\text { PM:SG2.SP2 }\end{array}$ \\
\hline PM-M2 & $\begin{array}{l}\text { percentage of staff-service dependency } \\
\text { conflicts with no mitigation plan }\end{array}$ & $\begin{array}{l}\text { asset-service } \\
\text { dependencies; } \\
\text { risk mitigation }\end{array}$ & impl & derived & $\begin{array}{l}\text { ADM:SG2.SP2 } \\
\text { PM:SG2.SP1 } \\
\text { PM:SG2.SP2 }\end{array}$ \\
\hline PM-M3 & $\begin{array}{l}\text { number of changes to service continuity } \\
\text { plans as a result of staff changes }\end{array}$ & $\begin{array}{l}\text { asset change } \\
\text { management; } \\
\text { SC plans }\end{array}$ & impl & $\begin{array}{l}\text { base of } \\
\text { type } \\
\text { count }\end{array}$ & ADM:SG3.SP2 \\
\hline PM-M4 & $\begin{array}{l}\text { percentage of staff and managers that } \\
\text { are designated as vital }\end{array}$ & $\begin{array}{l}\text { asset inventory; } \\
\text { vital staff; vital } \\
\text { managers }\end{array}$ & impl & derived & $\begin{array}{l}\text { ADM:SG1.SP1 } \\
\text { PM:SG1.SP1 }\end{array}$ \\
\hline PM-M5 & $\begin{array}{l}\text { elapsed time since the list of vital staff } \\
\text { has been reviewed and reconciled with } \\
\text { service continuity plans }\end{array}$ & $\begin{array}{l}\text { asset inventory; } \\
\text { vital staff }\end{array}$ & impl & $\begin{array}{l}\text { base of } \\
\text { type } \\
\text { schedule }\end{array}$ & PM:SG1.SP1 \\
\hline PM-M6 & $\begin{array}{l}\text { percentage of vital staff for which some } \\
\text { form of risk assessment of staff availabili- } \\
\text { ty has not been performed and docu- } \\
\text { mented (per policy or other guideline) } \\
\text { within the specified timeframe }\end{array}$ & asset risk & impl & derived & PM:SG2.SP1 \\
\hline PM-M7 & $\begin{array}{l}\text { percentage of vital staff availability risks } \\
\text { that have not been assigned to a respon- } \\
\text { sible party for action, tracking, and clo- } \\
\text { sure }\end{array}$ & asset risk & impl & derived & PM:SG2.SP2 \\
\hline PM-M8 & $\begin{array}{l}\text { percentage of vital staff availability risks } \\
\text { with a disposition of "mitigate or control" } \\
\text { that do not have a defined mitigation plan }\end{array}$ & asset risk & impl & derived & PM:SG2.SP2 ${ }^{39}$ \\
\hline
\end{tabular}

$39 \quad$ SG3.SP2 subpractice 7 states, "Collect performance measures on the risk management process." No such measures are included here in PM; refer to the RISK PA. 


\begin{tabular}{|c|c|c|c|c|c|}
\hline ID & Measure & $\begin{array}{l}\text { Type of } \\
\text { Information }\end{array}$ & $\begin{array}{l}\text { Measure } \\
\text { Type }\end{array}$ & $\begin{array}{l}\text { Base or } \\
\text { Derived }\end{array}$ & $\begin{array}{l}\text { Applicable } \\
\text { SG.SP }\end{array}$ \\
\hline PM-M9 & $\begin{array}{l}\text { percentage of vital staff availability risks } \\
\text { with a "mitigate or control" disposition } \\
\text { that are not effectively mitigated by their } \\
\text { mitigation plans }\end{array}$ & asset risk & impl & derived & PM:SG2.SP2 \\
\hline $\begin{array}{l}\text { PM- } \\
\text { M10 }\end{array}$ & $\begin{array}{l}\text { percentage of realized risks on the avail- } \\
\text { ability of vital staff that have exceeded } \\
\text { established risk parameters }\end{array}$ & asset risk & effectiveness & derived & PM:SG2.SP2 \\
\hline $\begin{array}{l}\text { PM- } \\
\text { M11 }\end{array}$ & $\begin{array}{l}\text { percentage of vital staff who do not have } \\
\text { redundancy plans }\end{array}$ & $\begin{array}{l}\text { vital staff; re- } \\
\text { dundancy plans }\end{array}$ & impl & derived & PM:SG3.SP1 \\
\hline $\begin{array}{l}\text { PM- } \\
\text { M12 }\end{array}$ & $\begin{array}{l}\text { cost required to address training gaps for } \\
\text { those designated as backups and re- } \\
\text { placements for vital staff }\end{array}$ & $\begin{array}{l}\text { vital staff; train- } \\
\text { ing gaps }\end{array}$ & impl & $\begin{array}{l}\text { base of } \\
\text { type cost }\end{array}$ & PM:SG3.SP1 \\
\hline $\begin{array}{l}\text { PM- } \\
\text { M13 }\end{array}$ & $\begin{array}{l}\text { elapsed time required to address training } \\
\text { gaps for those designated as backups } \\
\text { and replacements for vital staff }\end{array}$ & $\begin{array}{l}\text { vital staff; train- } \\
\text { ing gaps }\end{array}$ & impl & $\begin{array}{l}\text { base of } \\
\text { type } \\
\text { schedule }\end{array}$ & PM:SG3.SP1 \\
\hline $\begin{array}{l}\text { PM- } \\
\text { M14 }\end{array}$ & $\begin{array}{l}\text { effort required to address training gaps } \\
\text { for those designated as backups and } \\
\text { replacements for vital staff }\end{array}$ & $\begin{array}{l}\text { vital staff; train- } \\
\text { ing gaps }\end{array}$ & impl & $\begin{array}{l}\text { base of } \\
\text { type } \\
\text { effort }\end{array}$ & PM:SG3.SP1 \\
\hline $\begin{array}{l}\text { PM- } \\
\text { M15 }\end{array}$ & $\begin{array}{l}\text { percentage of vital staff available (on } \\
\text { hand) to conduct service continuity } \\
\text { planned exercises and tests (versus } \\
\text { those needed) }\end{array}$ & $\begin{array}{l}\text { vital staff; SC } \\
\text { tests }\end{array}$ & impl & derived & SC:SG5.SP3 \\
\hline $\begin{array}{l}\text { PM- } \\
\text { M16 }\end{array}$ & $\begin{array}{l}\text { percentage of vital staff not covered by a } \\
\text { service continuity plan }\end{array}$ & $\begin{array}{l}\text { vital staff; SC } \\
\text { plans }\end{array}$ & impl & derived & PM:SG3.SP3 \\
\hline $\begin{array}{l}\text { PM- } \\
\text { M17 }\end{array}$ & $\begin{array}{l}\text { percentage of vital staff who have not } \\
\text { been trained for redeployment }\end{array}$ & $\begin{array}{l}\text { vital staff; SC } \\
\text { plans }\end{array}$ & impl & derived & PM:SG3.SP3 \\
\hline $\begin{array}{l}\text { PM- } \\
\text { M18 }\end{array}$ & $\begin{array}{l}\text { percentage of vital managers who do not } \\
\text { have succession plans }\end{array}$ & $\begin{array}{l}\text { vital managers; } \\
\text { succession } \\
\text { plans }\end{array}$ & impl & derived & PM:SG3.SP2 \\
\hline $\begin{array}{l}\text { PM- } \\
\text { M19 }\end{array}$ & $\begin{array}{l}\text { number of reports to public authorities } \\
\text { regarding the loss of a vital higher level } \\
\text { manager }\end{array}$ & vital managers & impl & $\begin{array}{l}\text { base of } \\
\text { type } \\
\text { count }\end{array}$ & none \\
\hline $\begin{array}{l}\text { PM- } \\
\text { M20 }\end{array}$ & $\begin{array}{l}\text { percentage of first responders who do } \\
\text { not have appropriate credentials }\end{array}$ & first responders & impl & derived & PM:SG3.SP3 \\
\hline $\begin{array}{l}\text { PM- } \\
\text { M21 }\end{array}$ & $\begin{array}{l}\text { percentage of service continuity plans } \\
\text { that do not include plans to support staff } \\
\text { who are deployed during disruptive } \\
\text { events }\end{array}$ & $\begin{array}{l}\text { vital staff; SC } \\
\text { plans }\end{array}$ & impl & derived & $\begin{array}{l}\text { PM:SG3.SP4 } \\
\text { SC:SG3.SP2 }\end{array}$ \\
\hline $\begin{array}{l}\text { PM- } \\
\text { M22 }\end{array}$ & $\begin{array}{l}\text { percentage of service continuity plans } \\
\text { that do not include plans for transitioning } \\
\text { staff back to the workplace (return to } \\
\text { work) }\end{array}$ & $\begin{array}{l}\text { vital staff; SC } \\
\text { plans }\end{array}$ & impl & derived & $\begin{array}{l}\text { PM:SG3.SP5 } \\
\text { SC:SG3.SP2 }\end{array}$ \\
\hline $\begin{array}{l}\text { PM- } \\
\text { M23 }\end{array}$ & $\begin{array}{l}\text { number of people availability risks re- } \\
\text { ferred to the risk management process }\end{array}$ & $\begin{array}{l}\text { vital staff; vital } \\
\text { managers; risk } \\
\text { identification }\end{array}$ & impl & $\begin{array}{l}\text { base of } \\
\text { type } \\
\text { count }\end{array}$ & PM:SG2.SP1 \\
\hline
\end{tabular}

\section{Risk Management (RISK)}

The purpose of Risk Management is to identify, analyze, and mitigate risks to organizational assets that could adversely affect the operation and delivery of services.

\section{Summary of Specific Goals and Practices}

RISK:SG1 Prepare for Risk Management

RISK:SG1.SP1 Determine Risk Sources and Categories 
RISK:SG1.SP2 Establish an Operational Risk Management Strategy

RISK:SG2 Establish Risk Parameters and Focus

RISK:SG2.SP1 Define Risk Parameters

RISK:SG2.SP2 Establish Risk Measurement Criteria

RISK:SG3 Identify Risk

RISK:SG3.SP1 Identify Asset-Level Risks

RISK:SG3.SP2 Identify Service-Level Risks

RISK:SG4 Analyze Risk

RISK:SG4.SP1 Evaluate Risk

RISK:SG4.SP2 Categorize and Prioritize Risk

RISK:SG4.SP3 Assign Risk Disposition

RISK:SG5 Mitigate and Control Risk

RISK:SG5.SP1 Develop Risk Mitigation Plans

RISK:SG5.SP2 Implement Risk Strategies

RISK:SG6 Use Risk Information to Manage Resilience

RISK:SG6.SP1 Review and Adjust Strategies to Protect Assets and Services

RISK:SG6.SP2 Review and Adjust Strategies to Sustain Services

Measures

\begin{tabular}{|c|c|c|c|c|c|}
\hline ID & Measure & $\begin{array}{l}\text { Type of } \\
\text { Information }\end{array}$ & $\begin{array}{l}\text { Measure } \\
\text { Type }\end{array}$ & $\begin{array}{l}\text { Base vs. } \\
\text { Derived }\end{array}$ & $\begin{array}{l}\text { Applicable } \\
\text { SG.SP }\end{array}$ \\
\hline $\begin{array}{l}\text { RISK- } \\
\text { M1 }\end{array}$ & $\begin{array}{l}\text { number of internal operational risk sources } \\
\text { identified }\end{array}$ & risk planning & impl & $\begin{array}{l}\text { base of type } \\
\text { count }\end{array}$ & RISK:SG1.SP1 \\
\hline $\begin{array}{l}\text { RISK- } \\
\text { M2 }\end{array}$ & $\begin{array}{l}\text { number of external operational risk sources } \\
\text { identified }\end{array}$ & risk planning & impl & $\begin{array}{l}\text { base of type } \\
\text { count }\end{array}$ & RISK:SG1.SP1 \\
\hline $\begin{array}{l}\text { RISK- } \\
\text { M3 }\end{array}$ & $\begin{array}{l}\text { number of operational risk sources that are } \\
\text { not addressed by process policies or other } \\
\text { mitigating activities }\end{array}$ & risk sources & $\begin{array}{l}\text { effec- } \\
\text { tiveness }\end{array}$ & $\begin{array}{l}\text { base of type } \\
\text { count }\end{array}$ & RISK:SG1.SP1 \\
\hline $\begin{array}{l}\text { RISK- } \\
\text { M4 }\end{array}$ & number of risk categories defined & risk planning & impl & $\begin{array}{l}\text { base of type } \\
\text { count }\end{array}$ & RISK:SG1.SP1 \\
\hline $\begin{array}{l}\text { RISK- } \\
\text { M5 }\end{array}$ & $\begin{array}{l}\text { elapsed time since validation of risk catego- } \\
\text { ries performed }\end{array}$ & risk planning & impl & $\begin{array}{l}\text { base of type } \\
\text { schedule }\end{array}$ & RISK:SG1.SP1 \\
\hline $\begin{array}{l}\text { RISK- } \\
\text { M6 }\end{array}$ & $\begin{array}{l}\text { percentage of repeat audit findings related to } \\
\text { operational risk management }\end{array}$ & risk strategy & impl & derived & RISK:SG1.SP2 \\
\hline $\begin{array}{l}\text { RISK- } \\
\text { M7 }\end{array}$ & $\begin{array}{l}\text { number of operational risks referred to the } \\
\text { organization's enterprise risk management } \\
\text { process }\end{array}$ & risk strategy & impl & $\begin{array}{l}\text { base of type } \\
\text { count }\end{array}$ & RISK:SG1.SP2 \\
\hline $\begin{array}{l}\text { RISK- } \\
\text { M8 }\end{array}$ & number of risk parameters defined & risk strategy & impl & $\begin{array}{l}\text { base of type } \\
\text { count }\end{array}$ & RISK:SG2.SP1 \\
\hline $\begin{array}{l}\text { RISK- } \\
\text { M9 }\end{array}$ & $\begin{array}{l}\text { elapsed time since validation of risk parame- } \\
\text { ters performed }\end{array}$ & risk strategy & impl & $\begin{array}{l}\text { base of type } \\
\text { schedule }\end{array}$ & RISK:SG2.SP1 \\
\hline $\begin{array}{l}\text { RISK- } \\
\text { M10 }\end{array}$ & number of risk criteria defined & risk strategy & impl & $\begin{array}{l}\text { base of type } \\
\text { count }\end{array}$ & RISK:SG2.SP2 \\
\hline $\begin{array}{l}\text { RISK- } \\
\text { M11 }\end{array}$ & $\begin{array}{l}\text { elapsed time since validation of risk criteria } \\
\text { performed }\end{array}$ & risk strategy & impl & $\begin{array}{l}\text { base of type } \\
\text { schedule }\end{array}$ & RISK:SG2.SP2 \\
\hline
\end{tabular}




\begin{tabular}{|c|c|c|c|c|c|}
\hline ID & Measure & $\begin{array}{l}\text { Type of } \\
\text { Information }\end{array}$ & $\begin{array}{l}\text { Measure } \\
\text { Type }\end{array}$ & $\begin{array}{l}\text { Base vs. } \\
\text { Derived }\end{array}$ & $\begin{array}{l}\text { Applicable } \\
\text { SG.SP }\end{array}$ \\
\hline $\begin{array}{l}\text { RISK- } \\
\text { M12 }\end{array}$ & $\begin{array}{l}\text { elapsed time since risk assessment per- } \\
\text { formed }\end{array}$ & asset risk & impl & $\begin{array}{l}\text { base of type } \\
\text { schedule }\end{array}$ & RISK:SG3.SP1 \\
\hline $\begin{array}{l}\text { RISK- } \\
\text { M13 }\end{array}$ & $\begin{array}{l}\text { elapsed time since business impact analysis } \\
\text { performed }\end{array}$ & asset risk & impl & $\begin{array}{l}\text { base of type } \\
\text { schedule }\end{array}$ & RISK:SG3.SP1 \\
\hline $\begin{array}{l}\text { RISK- } \\
\text { M14 }\end{array}$ & $\begin{array}{l}\text { percentage of assets for which some form of } \\
\text { risk assessment has not been performed and } \\
\text { documented (per policy or other guideline) } \\
\text { within the specified timeframe }\end{array}$ & $\begin{array}{l}\text { risk assess- } \\
\text { ment }\end{array}$ & impl & derived & RISK:SG3.SP1 \\
\hline $\begin{array}{l}\text { RISK- } \\
\text { M15 }\end{array}$ & $\begin{array}{l}\text { percentage of services for which some form } \\
\text { of risk assessment of associated assets has } \\
\text { not been performed and documented (per } \\
\text { policy or other guideline) }\end{array}$ & $\begin{array}{l}\text { risk assess- } \\
\text { ment }\end{array}$ & impl & derived & RISK:SG3.SP2 \\
\hline $\begin{array}{l}\text { RISK- } \\
\text { M16 }\end{array}$ & $\begin{array}{l}\text { confidence factor that all risks that need to } \\
\text { be identified have been identified (refer to } \\
\text { template in [Allen 2010]) }\end{array}$ & $\begin{array}{l}\text { risk identifi- } \\
\text { cation }\end{array}$ & $\begin{array}{l}\text { effec- } \\
\text { tiveness }\end{array}$ & derived & $\begin{array}{l}\text { RISK:SG3.SP1 } \\
\text { RISK:SG3.SP2 }\end{array}$ \\
\hline $\begin{array}{l}\text { RISK- } \\
\text { M17 }\end{array}$ & $\begin{array}{l}\text { change in number of identified risks that } \\
\text { exceed risk parameters and measurement } \\
\text { criteria }\end{array}$ & $\begin{array}{l}\text { risk identifi- } \\
\text { cation; risk } \\
\text { valuation }\end{array}$ & impl & derived & $\begin{array}{l}\text { RISK:SG3.SP1 } \\
\text { RISK:SG3.SP2 } \\
\text { RISK:SG4.SP2 }\end{array}$ \\
\hline $\begin{array}{l}\text { RISK- } \\
\text { M18 }\end{array}$ & $\begin{array}{l}\text { percentage of risks for which the impact } \\
\text { (refer to RISK:SG2.SP2) has not been cha- } \\
\text { racterized (qualitative, quantitative) }\end{array}$ & risk valuation & impl & derived & RISK:SG4.SP1 \\
\hline $\begin{array}{l}\text { RISK- } \\
\text { M19 }\end{array}$ & $\begin{array}{l}\text { percentage of risks that have not been cate- } \\
\text { gorized and prioritized }\end{array}$ & $\begin{array}{l}\text { risk categori- } \\
\text { zation; risk } \\
\text { prioritization }\end{array}$ & impl & derived & RISK:SG4.SP2 \\
\hline $\begin{array}{l}\text { RISK- } \\
\text { M20 }\end{array}$ & $\begin{array}{l}\text { percentage of risks that have been characte- } \\
\text { rized as "high" impact according to risk pa- } \\
\text { rameters (refer to RISK:SG2) }\end{array}$ & risk valuation & impl & derived & RISK:SG4.SP1 \\
\hline $\begin{array}{l}\text { RISK- } \\
\text { M21 }\end{array}$ & $\begin{array}{l}\text { percentage of risks that exceed established } \\
\text { risk parameters and measurement criteria, } \\
\text { by risk category }\end{array}$ & $\begin{array}{l}\text { risk valua- } \\
\text { tion; risk } \\
\text { categoriza- } \\
\text { tion }\end{array}$ & impl & derived & $\begin{array}{l}\text { RISK:SG4.SP1 } \\
\text { RISK:SG4.SP2 }\end{array}$ \\
\hline $\begin{array}{l}\text { RISK- } \\
\text { M22 }\end{array}$ & $\begin{array}{l}\text { percentage of risks that do not have a docu- } \\
\text { mented and approved risk disposition }\end{array}$ & $\begin{array}{l}\text { risk disposi- } \\
\text { tion }\end{array}$ & impl & derived & RISK:SG4.SP3 \\
\hline $\begin{array}{l}\text { RISK- } \\
\text { M23 }\end{array}$ & $\begin{array}{l}\text { percentage of risks that have not been as- } \\
\text { signed to a responsible party for action, } \\
\text { tracking, and closure }\end{array}$ & $\begin{array}{l}\text { risk mitiga- } \\
\text { tion }\end{array}$ & impl & derived & RISK:SG5.SP1 \\
\hline $\begin{array}{l}\text { RISK- } \\
\text { M24 }\end{array}$ & $\begin{array}{l}\text { percentage of previously identified risks that } \\
\text { have converted from any other risk disposi- } \\
\text { tion to a risk disposition of "mitigate or con- } \\
\text { trol" }\end{array}$ & $\begin{array}{l}\text { risk disposi- } \\
\text { tion }\end{array}$ & impl & derived & RISK:SG4.SP3 \\
\hline $\begin{array}{l}\text { RISK- } \\
\text { M25 }\end{array}$ & $\begin{array}{l}\text { percentage of risks with a disposition of "mi- } \\
\text { tigate or control" that do not have a defined } \\
\text { mitigation plan }\end{array}$ & $\begin{array}{l}\text { risk disposi- } \\
\text { tion; risk } \\
\text { mitigation }\end{array}$ & impl & derived & RISK:SG5.SP1 \\
\hline $\begin{array}{l}\text { RISK- } \\
\text { M26 }\end{array}$ & $\begin{array}{l}\text { percentage of assets for which a mitigation } \\
\text { plan has been implemented to mitigate risks } \\
\text { as necessary and to maintain these risks } \\
\text { within acceptable risk parameters }\end{array}$ & $\begin{array}{l}\text { risk mitiga- } \\
\text { tion; risk } \\
\text { status }\end{array}$ & impl & derived & $\begin{array}{l}\text { RISK:SG5.SP1 } \\
\text { RISK:SG5.SP2 }\end{array}$ \\
\hline $\begin{array}{l}\text { RISK- } \\
\text { M27 }\end{array}$ & $\begin{array}{l}\text { percentage of services with an implemented } \\
\text { mitigation plan }\end{array}$ & $\begin{array}{l}\text { risk mitiga- } \\
\text { tion; risk } \\
\text { status }\end{array}$ & impl & derived & $\begin{array}{l}\text { RISK:SG5.SP1 } \\
\text { RISK:SG5.SP2 }\end{array}$ \\
\hline $\begin{array}{l}\text { RISK- } \\
\text { M28 }\end{array}$ & $\begin{array}{l}\text { percentage of risks with a "mitigate or con- } \\
\text { trol" disposition with mitigations }{ }^{40} \text { that are not } \\
\text { yet started }\end{array}$ & $\begin{array}{l}\text { risk mitiga- } \\
\text { tion; risk } \\
\text { status }\end{array}$ & impl & derived & $\begin{array}{l}\text { RISK:SG5.SP2 } \\
\text { RISK:SG6.SP1 } \\
\text { RISK:SG6.SP2 }\end{array}$ \\
\hline
\end{tabular}

40 Including controls and updates to SC plans 


\begin{tabular}{|l|l|l|l|l|l|}
\hline ID & Measure & $\begin{array}{l}\text { Type of } \\
\text { Information }\end{array}$ & $\begin{array}{l}\text { Measure } \\
\text { Type }\end{array}$ & $\begin{array}{l}\text { Base vs. } \\
\text { Derived }\end{array}$ & $\begin{array}{l}\text { Applicable } \\
\text { SG.SP }\end{array}$ \\
\hline $\begin{array}{l}\text { RISK- } \\
\text { M29 }\end{array}$ & $\begin{array}{l}\text { percentage of risks with a "mitigate or con- } \\
\text { trol" disposition with mitigations that are in } \\
\text { progress (vs. completely implemented) }\end{array}$ & $\begin{array}{l}\text { risk mitiga- } \\
\text { tion; risk } \\
\text { status }\end{array}$ & impl & derived & $\begin{array}{l}\text { RISK:SG5.SP2 } \\
\text { RISK:SG6.SP1 } \\
\text { RISK:SG6.SP2 }\end{array}$ \\
\hline $\begin{array}{l}\text { RISK- } \\
\text { M30 }\end{array}$ & $\begin{array}{l}\text { percentage of risks with a "mitigate or con- } \\
\text { trol" disposition that are not effectively miti- } \\
\text { gated by their mitigation plans }\end{array}$ & $\begin{array}{l}\text { risk mitiga- } \\
\text { tion; risk } \\
\text { status }\end{array}$ & $\begin{array}{l}\text { effec- } \\
\text { tiveness }\end{array}$ & $\begin{array}{l}\text { base of type } \\
\text { ordinal/ratio }\end{array}$ & RISK:SG5.SP2 \\
\hline $\begin{array}{l}\text { RISK- } \\
\text { M31 }\end{array}$ & $\begin{array}{l}\text { percentage of open risks that have not been } \\
\text { tracked to closure }\end{array}$ & risk status & impl & derived & RISK:SG5.SP2 \\
\hline $\begin{array}{l}\text { RISK- } \\
\text { M32 }\end{array}$ & $\begin{array}{l}\text { percentage of risks with a disposition of "mi- } \\
\text { tigate or control" that have a defined mitiga- } \\
\text { tion plan but whose status is not regularly } \\
\text { reported (per policy or other guideline) }\end{array}$ & risk status & impl & derived & RISK:SG5.SP2 \\
\hline $\begin{array}{l}\text { RISK- } \\
\text { M33 }\end{array}$ & $\begin{array}{l}\text { percentage of realized risks that exceed } \\
\text { established risk parameters }\end{array}$ & risk status & $\begin{array}{l}\text { effec- } \\
\text { tiveness }\end{array}$ & derived & $\begin{array}{l}\text { refer to compa- } \\
\text { rable measures } \\
\text { in EC, EXD, } \\
\text { IMC, KIM, TM }\end{array}$ \\
\hline $\begin{array}{l}\text { RISK- } \\
\text { M34 }\end{array}$ & $\begin{array}{l}\text { elapsed time since risks with the following } \\
\text { dispositions were last reviewed and disposi- } \\
\text { tion confirmed: avoid, accept, monitor, re- } \\
\text { search or defer, transfer }\end{array}$ & risk status & impl & $\begin{array}{l}\text { base of type } \\
\text { schedule }\end{array}$ & RISK:SG5.SP2 \\
\hline
\end{tabular}

\section{Resilience Requirement Development (RRD)}

The purpose of Resilience Requirements Development is to identify, document, and analyze the operational resilience requirements for high-value services and related assets.

\section{Summary of Specific Goals and Practices}

RRD:SG1 Identify Enterprise Requirements

RRD:SG1.SP1 Establish Enterprise Resilience Requirements

RRD:SG2 Develop Service Requirements

RRD:SG2.SP1 Establish Asset Resilience Requirements

RRD:SG2.SP2 Assign Enterprise Resilience Requirements to Services

RRD:SG3 Analyze and Validate Requirements

RRD:SG3.SP1 Establish a Definition of Required Functionality

RRD:SG3.SP2 Analyze Resilience Requirements

RRD:SG3.SP3 Validate Resilience Requirements

\section{Measures}

\begin{tabular}{|l|l|l|l|l|l|}
\hline ID & Measure & $\begin{array}{l}\text { Type of } \\
\text { Information }\end{array}$ & $\begin{array}{l}\text { Measure } \\
\text { Type }\end{array}$ & $\begin{array}{l}\text { Base or } \\
\text { Derived }\end{array}$ & $\begin{array}{l}\text { Applicable } \\
\text { SG.SP }\end{array}$ \\
\hline $\begin{array}{l}\text { RRD- } \\
\text { M1 }\end{array}$ & $\begin{array}{l}\text { percentage of enterprise requirements that } \\
\text { have been communicated to all organizational } \\
\text { units and lines of business }\end{array}$ & $\begin{array}{l}\text { enterprise } \\
\text { requirements }\end{array}$ & impl & derived & RRD:SG1.SP1 \\
\hline $\begin{array}{l}\text { RRD- } \\
\text { M2 }\end{array}$ & $\begin{array}{l}\text { percentage of services with incomplete or no } \\
\text { stated requirements }\end{array}$ & $\begin{array}{l}\text { service re- } \\
\text { quirements }\end{array}$ & impl & derived & $\begin{array}{l}\text { RRD:SG2.SP1 } \\
\text { RRD:SG2.SP2 }\end{array}$ \\
\hline
\end{tabular}

41 May want to specifically categorize by source of realized risk that is of greatest interest such as incidents, control gaps, non-compliance, vulnerabilities, disruptions in continuity, etc. 


\begin{tabular}{|c|c|c|c|c|c|}
\hline ID & Measure & $\begin{array}{l}\text { Type of } \\
\text { Information }\end{array}$ & $\begin{array}{l}\text { Measure } \\
\text { Type }\end{array}$ & $\begin{array}{l}\text { Base or } \\
\text { Derived }\end{array}$ & $\begin{array}{l}\text { Applicable } \\
\text { SG.SP }\end{array}$ \\
\hline $\begin{array}{l}\text { RRD- } \\
\text { M3 }\end{array}$ & $\begin{array}{l}\text { percentage of assets with incomplete or no } \\
\text { stated requirements }\end{array}$ & $\begin{array}{l}\text { asset require- } \\
\text { ments }\end{array}$ & impl & derived & $\begin{array}{l}\text { RRD:SG2.SP1 } \\
\text { RRD:SG2.SP2 }\end{array}$ \\
\hline $\begin{array}{l}\text { RRD- } \\
\text { M4 }\end{array}$ & $\begin{array}{l}\text { percentage of service owners participating in } \\
\text { the development of requirements (should be } \\
100 \% \text { ) }\end{array}$ & $\begin{array}{l}\text { service re- } \\
\text { quirements }\end{array}$ & impl & derived & $\begin{array}{l}\text { RRD:SG2.SP1 } \\
\text { RRD:SG2.SP2 }\end{array}$ \\
\hline $\begin{array}{l}\text { RRD- } \\
\text { M5 }\end{array}$ & $\begin{array}{l}\text { percentage of asset owners participating in } \\
\text { the development of requirements (should be } \\
100 \% \text { ) }\end{array}$ & $\begin{array}{l}\text { asset require- } \\
\text { ments }\end{array}$ & impl & derived & $\begin{array}{l}\text { RRD:SG2.SP1 } \\
\text { RRD:SG2.SP2 }\end{array}$ \\
\hline $\begin{array}{l}\text { RRD- } \\
\text { M6 }\end{array}$ & $\begin{array}{l}\text { percentage of documented requirements that } \\
\text { have not been implemented }{ }^{42}\end{array}$ & $\begin{array}{l}\text { enterprise, } \\
\text { service, and } \\
\text { asset require- } \\
\text { ments }\end{array}$ & $\begin{array}{l}\text { impl; } \\
\text { possibly } \\
\text { effec- } \\
\text { tiveness }\end{array}$ & derived & none \\
\hline $\begin{array}{l}\text { RRD- } \\
\text { M7 }\end{array}$ & $\begin{array}{l}\text { percentage of assets for which the required } \\
\text { level of functionality of the asset is not docu- } \\
\text { mented for all services it supports }\end{array}$ & $\begin{array}{l}\text { asset require- } \\
\text { ments }\end{array}$ & impl & derived & RRD:SG3.SP1 \\
\hline $\begin{array}{l}\text { RRD- } \\
\text { M8 }\end{array}$ & $\begin{array}{l}\text { percentage of assets with requirements revi- } \\
\text { sions due to: } \\
\text { - conflicts resulting from associations with } \\
\text { multiple services } \\
\text { - requirements deficiencies } \\
\text { - enterprise requirements } \\
\text { - requirements gaps }\end{array}$ & $\begin{array}{l}\text { asset require- } \\
\text { ments }\end{array}$ & impl & derived & $\begin{array}{l}\text { RRD:SG3.SP2 } \\
\text { RRD:SG3.SP3 }\end{array}$ \\
\hline $\begin{array}{l}\text { RRD- } \\
\text { M9 }\end{array}$ & $\begin{array}{l}\text { percentage of asset requirements conflicts for } \\
\text { which mitigation plans have been developed } \\
\text { but not implemented }\end{array}$ & $\begin{array}{l}\text { asset require- } \\
\text { ments }\end{array}$ & impl & derived & RRD:SG3.SP2 \\
\hline $\begin{array}{l}\text { RRD- } \\
\text { M10 }\end{array}$ & $\begin{array}{l}\text { percentage of requirements that have not } \\
\text { been analyzed to identify conflicts and inter- } \\
\text { dependencies }\end{array}$ & $\begin{array}{l}\text { asset require- } \\
\text { ments }\end{array}$ & impl & derived & RRD:SG3.SP2 \\
\hline $\begin{array}{l}\text { RRD- } \\
\text { M11 }\end{array}$ & $\begin{array}{l}\text { percentage of requirements whose adequacy } \\
\text { has not been validated }\end{array}$ & $\begin{array}{l}\text { asset require- } \\
\text { ments }\end{array}$ & impl & derived & RRD:SG3.SP3 \\
\hline $\begin{array}{l}\text { RRD- } \\
\text { M12 }\end{array}$ & $\begin{array}{l}\text { elapsed time between identification of new } \\
\text { assets and the development of requirements } \\
\text { for these assets (mean, median) }\end{array}$ & $\begin{array}{l}\text { asset require- } \\
\text { ments }\end{array}$ & impl & $\begin{array}{l}\text { base of } \\
\text { type } \\
\text { schedule }\end{array}$ & $\begin{array}{l}\text { ADM:SG3.SP2 } \\
\text { RRD:SG2.SP1 }\end{array}$ \\
\hline $\begin{array}{l}\text { RRD- } \\
\text { M13 }\end{array}$ & $\begin{array}{l}\text { costs of developing, analyzing, validating, } \\
\text { documenting, and tracking requirements }\end{array}$ & $\begin{array}{l}\text { enterprise, } \\
\text { service, and } \\
\text { asset require- } \\
\text { ments }\end{array}$ & impl & $\begin{array}{l}\text { base of } \\
\text { type cost }\end{array}$ & none \\
\hline $\begin{array}{l}\text { RRD- } \\
\text { M14 }\end{array}$ & $\begin{array}{l}\text { percentage of service continuity test failures } \\
\text { caused by incorrect or missing requirements }\end{array}$ & $\begin{array}{l}\text { service re- } \\
\text { quirements }\end{array}$ & $\begin{array}{l}\text { effec- } \\
\text { tiveness }\end{array}$ & derived & none \\
\hline $\begin{array}{l}\text { RRD- } \\
\text { M15 }\end{array}$ & $\begin{array}{l}\text { percentage of incidents caused by incorrect or } \\
\text { missing requirements }\end{array}$ & $\begin{array}{l}\text { asset require- } \\
\text { ments }\end{array}$ & $\begin{array}{l}\text { effec- } \\
\text { tiveness }\end{array}$ & derived & none \\
\hline
\end{tabular}

\section{Resilience Requirements Management (RRM)}

The purpose of Resilience Requirements Management is to manage the resilience requirements of high-value services and associated assets and to identify inconsistencies between these requirements and the activities that the organization performs to meet the requirements.

\section{Summary of Specific Goals and Practices}

RRM:SG1 Manage Requirements

RRM:SG1.SP1 Obtain an Understanding of Resilience Requirements

42 While included as an RRD measure of interest, implementation of requirements is covered in other PAs (enterprise - EF, RISK, etc.; service - EF, SC; asset - EC, KIM, PM, TM) 
RRM:SG1.SP2 Obtain Commitment to Resilience Requirements

RRM:SG1.SP3 Manage Resilience Requirements Changes

RRM:SG1.SP4 Maintain Traceability of Resilience Requirements

RRM:SG1.SP5 Identify Inconsistencies Between Resilience Requirements and Activities Performed to Meet the Requirements

\section{Measures}

\begin{tabular}{|c|c|c|c|c|c|}
\hline ID & Measure & $\begin{array}{l}\text { Type of } \\
\text { Information }\end{array}$ & $\begin{array}{l}\text { Measure } \\
\text { Type }\end{array}$ & $\begin{array}{l}\text { Base or } \\
\text { Derived }\end{array}$ & $\begin{array}{l}\text { Applicable } \\
\text { SG.SP }\end{array}$ \\
\hline $\begin{array}{l}\text { RRM- } \\
\text { M1 }\end{array}$ & $\begin{array}{l}\text { percentage of assets for which agreement } \\
\text { between asset owners and custodians on } \\
\text { asset requirements has not been reached }\end{array}$ & $\begin{array}{l}\text { asset require- } \\
\text { ments }\end{array}$ & impl & derived & RRM:SG1.SP1 \\
\hline $\begin{array}{l}\text { RRM- } \\
\text { M2 }\end{array}$ & $\begin{array}{l}\text { percentage of service level agreements } \\
\text { between asset owners and custodians that } \\
\text { are pending sign-off due to requirements } \\
\text { issues }\end{array}$ & $\begin{array}{l}\text { asset require- } \\
\text { ments }\end{array}$ & impl & derived & RRM:SG1.SP2 \\
\hline $\begin{array}{l}\text { RRM- } \\
\text { M3 }\end{array}$ & $\begin{array}{l}\text { percentage of asset custodians who accept } \\
\text { responsibility for implementing require- } \\
\text { ments, if applicable }\end{array}$ & $\begin{array}{l}\text { asset require- } \\
\text { ments }\end{array}$ & impl & derived & $\begin{array}{l}\text { RRM:SG1.SP1 } \\
\text { RRM:SG1.SP2 }\end{array}$ \\
\hline $\begin{array}{l}\text { RRM- } \\
\text { M4 }\end{array}$ & $\begin{array}{l}\text { percentage of documented, agreed-to re- } \\
\text { quirements that have not been imple- } \\
\text { mented }^{43} \text { as scheduled }\end{array}$ & $\begin{array}{l}\text { enterprise, ser- } \\
\text { vice, and asset } \\
\text { requirements }\end{array}$ & $\mathrm{impl}$ & derived & none \\
\hline $\begin{array}{l}\text { RRM- } \\
\text { M5 }\end{array}$ & $\begin{array}{l}\text { percentage of asset owners participating in } \\
\text { managing changes to requirements for the } \\
\text { assets they own }\end{array}$ & $\begin{array}{l}\text { changes to re- } \\
\text { quirements }\end{array}$ & impl & derived & $\begin{array}{l}\text { RRM:SG1.SP3 } \\
\text { also EC, KIM, } \\
\text { TM }\end{array}$ \\
\hline $\begin{array}{l}\text { RRM- } \\
\text { M6 }\end{array}$ & $\begin{array}{l}\text { number of approved requirements changes: } \\
\text { - by asset category or type } \\
\text { - by asset } \\
\text { - by service } \\
\text { - by change trigger and criteria }\end{array}$ & $\begin{array}{l}\text { changes to re- } \\
\text { quirements }\end{array}$ & impl & $\begin{array}{l}\text { base of } \\
\text { type } \\
\text { count }\end{array}$ & RRM:SG1.SP3 \\
\hline $\begin{array}{l}\text { RRM- } \\
\text { M7 }\end{array}$ & $\begin{array}{l}\text { number of unapproved requirements } \\
\text { changes }\end{array}$ & $\begin{array}{l}\text { changes to re- } \\
\text { quirements }\end{array}$ & $\begin{array}{l}\text { effective- } \\
\text { ness }\end{array}$ & $\begin{array}{l}\text { base of } \\
\text { type } \\
\text { count }\end{array}$ & RRM:SG1.SP3 \\
\hline $\begin{array}{l}\text { RRM- } \\
\text { M8 }\end{array}$ & $\begin{array}{l}\text { number of approved requirements changes } \\
\text { that have not been communicated to asset } \\
\text { custodians (via defined channels or SLAs) }\end{array}$ & $\begin{array}{l}\text { changes to re- } \\
\text { quirements }\end{array}$ & $\mathrm{impl}$ & $\begin{array}{l}\text { base of } \\
\text { type } \\
\text { count }\end{array}$ & RRM:SG1.SP3 \\
\hline $\begin{array}{l}\text { RRM- } \\
\text { M9 }\end{array}$ & $\begin{array}{l}\text { percentage of requirements change re- } \\
\text { quests whose disposition is pending beyond } \\
\text { schedule }\end{array}$ & $\begin{array}{l}\text { changes to re- } \\
\text { quirements }\end{array}$ & $\mathrm{impl}$ & derived & RRM:SG1.SP3 \\
\hline $\begin{array}{l}\text { RRM- } \\
\text { M10 }\end{array}$ & $\begin{array}{l}\text { percentage of approved requirements } \\
\text { changes whose implementation is pending } \\
\text { beyond schedule }\end{array}$ & $\begin{array}{l}\text { changes to re- } \\
\text { quirements }\end{array}$ & $\mathrm{impl}$ & derived & RRM:SG1.SP3 \\
\hline $\begin{array}{l}\text { RRM- } \\
\text { M11 }\end{array}$ & $\begin{array}{l}\text { percentage of requirements changes that } \\
\text { are not subject to the organization's change } \\
\text { control process }\end{array}$ & $\begin{array}{l}\text { changes to re- } \\
\text { quirements }\end{array}$ & impl & derived & RRM:SG1.SP3 \\
\hline $\begin{array}{l}\text { RRM- } \\
\text { M12 }\end{array}$ & $\begin{array}{l}\text { costs of analyzing, managing, documenting, } \\
\text { and tracking changes to requirements }\end{array}$ & $\begin{array}{l}\text { changes to re- } \\
\text { quirements }\end{array}$ & $\mathrm{impl}$ & $\begin{array}{l}\text { base of } \\
\text { type cost }\end{array}$ & $\begin{array}{l}\text { FRM:SG2.SP2 } \\
\text { RRM:SG1.SP3 }\end{array}$ \\
\hline $\begin{array}{l}\text { RRM- } \\
\text { M13 }\end{array}$ & $\begin{array}{l}\text { percentage of requirements that are not } \\
\text { traced to a source or origination (docu- } \\
\text { mented in the asset profile) }\end{array}$ & $\begin{array}{l}\text { requirements } \\
\text { traceability }\end{array}$ & impl & derived & RRM:SG1.SP4 \\
\hline
\end{tabular}

43 While included as an RRM measure of interest, actual implementation of requirements is covered in other PAs (enterprise - EF, RISK, etc.; service - EF, SC; asset - EC, KIM, PM, TM) 


\begin{tabular}{|l|l|l|l|l|l|}
\hline ID & Measure & $\begin{array}{l}\text { Type of } \\
\text { Information }\end{array}$ & $\begin{array}{l}\text { Measure } \\
\text { Type }\end{array}$ & $\begin{array}{l}\text { Base or } \\
\text { Derived }\end{array}$ & $\begin{array}{l}\text { Applicable } \\
\text { SG.SP }\end{array}$ \\
\hline $\begin{array}{l}\text { RRM- } \\
\text { M14 }\end{array}$ & $\begin{array}{l}\text { percentage of resilience activities that are } \\
\text { not traced to a requirement }\end{array}$ & $\begin{array}{l}\text { requirements } \\
\text { traceability }\end{array}$ & impl & derived & RRM:SG1.SP4 \\
\hline $\begin{array}{l}\text { RRM- } \\
\text { M15 }\end{array}$ & $\begin{array}{l}\text { number of inconsistencies detected be- } \\
\text { tween requirements and the activities in } \\
\text { place to satisfy the requirements }\end{array}$ & $\begin{array}{l}\text { enterprise, ser- } \\
\text { vice, and asset } \\
\text { requirements }\end{array}$ & impl & $\begin{array}{l}\text { base of } \\
\text { type } \\
\text { count }\end{array}$ & RRM:SG1.SP5 \\
\hline $\begin{array}{l}\text { RRM- } \\
\text { M16 }\end{array}$ & $\begin{array}{l}\text { number of corrective actions to align re- } \\
\text { quirements and the activities required to } \\
\text { satisfy them that are open beyond threshold } \\
\text { (as scheduled) }\end{array}$ & $\begin{array}{l}\text { enterprise, ser- } \\
\text { vice, and asset } \\
\text { requirements }\end{array}$ & impl & $\begin{array}{l}\text { base of } \\
\text { type } \\
\text { count }\end{array}$ & RRM:SG1.SP5 \\
\hline $\begin{array}{l}\text { RRM- } \\
\text { M17 }\end{array}$ & $\begin{array}{l}\text { elapsed time between major updates to } \\
\text { assets (such as being associated with a } \\
\text { new service) and updates to the require- } \\
\text { ments for these assets (mean, median) }\end{array}$ & $\begin{array}{l}\text { asset require- } \\
\text { ments }\end{array}$ & impl & $\begin{array}{l}\text { base of } \\
\text { type } \\
\text { schedule }\end{array}$ & $\begin{array}{l}\text { ADM:SG3.SP2 } \\
\text { RRM:SG1.SP3 }\end{array}$ \\
\hline
\end{tabular}

\section{Resilient Technical Solution Engineering (RTSE)}

The purpose of Resilient Technical Solution Engineering is to ensure that software and systems are developed to satisfy their resilience requirements.

\section{Summary of Specific Goals and Practices}

RTSE:SG1 Establish Guidelines for Resilient Technical Solution Development

RTSE:SG1.SP1 Identify General Guidelines

RTSE:SG1.SP2 Identify Requirements Guidelines

RTSE:SG1.SP3 Identify Architecture and Design Guidelines

RTSE:SG1.SP4 Identify Implementation Guidelines

RTSE:SG1.SP5 Identify Assembly and Integration Guidelines

RTSE:SG2 Develop Resilient Technical Solution Development Plans

RTSE:SG2.SP1 Select and Tailor Guidelines

RTSE:SG2.SP2 Integrate Selected Guidelines with a Defined Software and System Development Process

RTSE:SG3 Execute the Plan

RTSE:SG3.SP1 Monitor Execution of the Development Plan

RTSE:SG3.SP2 Release Resilient Technical Solutions into Production

\section{Measures}

\begin{tabular}{|l|l|l|l|l|l|}
\hline ID & Measure & $\begin{array}{l}\text { Type of } \\
\text { Information }\end{array}$ & $\begin{array}{l}\text { Measure } \\
\text { Type }\end{array}$ & $\begin{array}{l}\text { Base or } \\
\text { Derived }\end{array}$ & $\begin{array}{l}\text { Applicable } \\
\text { SG.SP }\end{array}$ \\
\hline $\begin{array}{l}\text { RTSE- } \\
\text { M1 }\end{array}$ & $\begin{array}{l}\text { percentage of software assets that have } \\
\text { been developed without resilience guide- } \\
\text { lines, by guideline type: } \\
\text { - general } \\
\text { - requirements } \\
\text { - architecture and design } \\
\text { - implementation } \\
\text { - assembly and integration }\end{array}$ & $\begin{array}{l}\text { resilience guide- } \\
\text { lines for software } \\
\text { development }\end{array}$ & $\begin{array}{l}\text { impl; pos- } \\
\text { sibly effec- } \\
\text { tiveness }\end{array}$ & derived & $\begin{array}{l}\text { RTSE:SG2.SP1 } \\
\text { Could also be } \\
\text { mapped to each } \\
\text { of the SG1 spe- } \\
\text { cific practices }\end{array}$ \\
\hline
\end{tabular}




\begin{tabular}{|c|c|c|c|c|c|}
\hline ID & Measure & $\begin{array}{l}\text { Type of } \\
\text { Information }\end{array}$ & $\begin{array}{l}\text { Measure } \\
\text { Type }\end{array}$ & $\begin{array}{l}\text { Base or } \\
\text { Derived }\end{array}$ & $\begin{array}{l}\text { Applicable } \\
\text { SG.SP }\end{array}$ \\
\hline $\begin{array}{l}\text { RTSE- } \\
\text { M2 }\end{array}$ & $\begin{array}{l}\text { percentage of software assets that have } \\
\text { been acquired without consideration of } \\
\text { resilience guidelines, by guideline type: } \\
\text { - general } \\
\text { - requirements } \\
\text { - architecture and design } \\
\text { - implementation } \\
\text { - assembly and integration }\end{array}$ & $\begin{array}{l}\text { resilience guide- } \\
\text { lines for software } \\
\text { acquisition }\end{array}$ & $\begin{array}{l}\text { impl; pos- } \\
\text { sibly effec- } \\
\text { tiveness }\end{array}$ & derived & $\begin{array}{l}\text { RTSE:SG2.SP1 } \\
\text { Could also be } \\
\text { mapped to each } \\
\text { of the SG1 spe- } \\
\text { cific practices }\end{array}$ \\
\hline $\begin{array}{l}\text { RTSE- } \\
\text { M3 }\end{array}$ & $\begin{array}{l}\text { percentage of software development staff } \\
\text { trained in the tailoring and use of resilience } \\
\text { guidelines, by guideline type: } \\
\text { - general } \\
\text { - requirements } \\
\text { - architecture and design } \\
\text { - implementation } \\
\text { - assembly and integration }\end{array}$ & $\begin{array}{l}\text { resilience guide- } \\
\text { lines for software } \\
\text { development }\end{array}$ & $\begin{array}{l}\text { impl; pos- } \\
\text { sibly effec- } \\
\text { tiveness }\end{array}$ & derived & $\begin{array}{l}\text { RTSE:SG2.SP1 } \\
\text { Could also be } \\
\text { mapped to each } \\
\text { of the SG1 spe- } \\
\text { cific practices }\end{array}$ \\
\hline $\begin{array}{l}\text { RTSE- } \\
\text { M4 }\end{array}$ & $\begin{array}{l}\text { life-cycle costs associated with implement- } \\
\text { ing each resilience guideline (time, staff } \\
\text { resources, and funding, including training) } \\
\text { or some meaningful collection of guidelines }\end{array}$ & $\begin{array}{l}\text { resilience guide- } \\
\text { line costs }\end{array}$ & impl & derived & $\begin{array}{l}\text { RTSE:SG2.SP1 } \\
\text { Could also be } \\
\text { mapped to each } \\
\text { of the SG1 spe- } \\
\text { cific practices }\end{array}$ \\
\hline $\begin{array}{l}\text { RTSE- } \\
\text { M5 }\end{array}$ & $\begin{array}{l}\text { percentage of resilience requirements not } \\
\text { satisfied by a specific software or system } \\
\text { asset }^{44} \\
\text { ranked in priority order (refer to RRD) } \\
\text { by life-cycle phase }\end{array}$ & $\begin{array}{l}\text { resilience re- } \\
\text { quirements }\end{array}$ & impl & derived & $\begin{array}{l}\text { RTSE:SG3.SP1 } \\
\text { RTSE:SG3.SP2 }\end{array}$ \\
\hline $\begin{array}{l}\text { RTSE- } \\
\text { M6 }\end{array}$ & $\begin{array}{l}\text { percentage of resilience requirements not } \\
\text { satisfied by a specific software or system } \\
\text { asset, where lack of satisfaction has been } \\
\text { identified as a residual risk to be managed }\end{array}$ & $\begin{array}{l}\text { resilience re- } \\
\text { quirements; risk } \\
\text { identification }\end{array}$ & impl & derived & $\begin{array}{l}\text { RTSE:SG3.SP1 } \\
\text { RTSE:SG3.SP2 }\end{array}$ \\
\hline $\begin{array}{l}\text { RTSE- } \\
\text { M7 }\end{array}$ & $\begin{array}{l}\text { number of defects and vulnerabilities above } \\
\text { threshold for a specific software or system } \\
\text { asset by life-cycle phase }\end{array}$ & $\begin{array}{l}\text { vulnerabilities } \\
\text { and defects }\end{array}$ & impl & $\begin{array}{l}\text { base of } \\
\text { type } \\
\text { count }\end{array}$ & RTSE:SG3.SP1 \\
\hline $\begin{array}{l}\text { RTSE- } \\
\text { M8 }\end{array}$ & $\begin{array}{l}\text { number of defects and vulnerabilities above } \\
\text { threshold for a specific software or system } \\
\text { asset where such defects and vulnerabilities } \\
\text { have documented mitigation plans }\end{array}$ & $\begin{array}{l}\text { vulnerabilities } \\
\text { and defects }\end{array}$ & impl & $\begin{array}{l}\text { base of } \\
\text { type } \\
\text { count }\end{array}$ & RTSE:SG3.SP1 \\
\hline $\begin{array}{l}\text { RTSE- } \\
\text { M9 }\end{array}$ & $\begin{array}{l}\text { number of defects and vulnerabilities above } \\
\text { threshold for a specific software or system } \\
\text { asset where such defects and vulnerabilities } \\
\text { have been identified as residual risks to be } \\
\text { managed }\end{array}$ & $\begin{array}{l}\text { vulnerabilities } \\
\text { and defects; risk } \\
\text { identification }\end{array}$ & impl & $\begin{array}{l}\text { base of } \\
\text { type } \\
\text { count }\end{array}$ & RTSE:SG3.SP1 \\
\hline $\begin{array}{l}\text { RTSE- } \\
\text { M10 }\end{array}$ & $\begin{array}{l}\text { number of defects and vulnerabilities above } \\
\text { threshold for a specific software or system } \\
\text { assets where the presence of such defects } \\
\text { and vulnerabilities is a result of not imple- } \\
\text { menting a resilience guideline }\end{array}$ & $\begin{array}{l}\text { vulnerabilities } \\
\text { and defects }\end{array}$ & impl & $\begin{array}{l}\text { base of } \\
\text { type } \\
\text { count }\end{array}$ & RTSE:SG3.SP1 \\
\hline $\begin{array}{l}\text { RTSE- } \\
\text { M11 }\end{array}$ & $\begin{array}{l}\text { percentage of software assets for which } \\
\text { some form of risk assessment has not been } \\
\text { performed and documented (per policy or } \\
\text { other resilience guidelines) and within the } \\
\text { specified time frame, by life-cycle phase }\end{array}$ & asset risk & impl & derived & $\begin{array}{l}\text { RTSE:SG2.SP1 } \\
\text { RTSE:SG2.SP2 } \\
\text { RTSE:SG3.SP1 }\end{array}$ \\
\hline
\end{tabular}

44 This presumes that criteria for satisfaction are well established, such as evidence associated with one or more assurance cases or the results of specific review milestones or selected test cases. 


\begin{tabular}{|c|c|c|c|c|c|}
\hline ID & Measure & $\begin{array}{l}\text { Type of } \\
\text { Information }\end{array}$ & $\begin{array}{l}\text { Measure } \\
\text { Type }\end{array}$ & $\begin{array}{l}\text { Base or } \\
\text { Derived }\end{array}$ & $\begin{array}{l}\text { Applicable } \\
\text { SG.SP }\end{array}$ \\
\hline $\begin{array}{l}\text { RTSE- } \\
\text { M12 }\end{array}$ & $\begin{array}{l}\text { percentage of system assets for which } \\
\text { some form of risk assessment has not been } \\
\text { performed and documented (per policy or } \\
\text { other resilience guidelines) and within the } \\
\text { specified time frame, by life-cycle phase }\end{array}$ & asset risk & impl & derived & $\begin{array}{l}\text { RTSE:SG2.SP1 } \\
\text { RTSE:SG2.SP2 } \\
\text { RTSE:SG3.SP1 }\end{array}$ \\
\hline $\begin{array}{l}\text { RTSE- } \\
\text { M13 }\end{array}$ & $\begin{array}{l}\text { number of unauthorized changes to soft- } \\
\text { ware assets, by life-cycle phase }\end{array}$ & $\begin{array}{l}\text { asset change } \\
\text { management }\end{array}$ & impl & $\begin{array}{l}\text { base of } \\
\text { type } \\
\text { count }\end{array}$ & RTSE:SG3.SP1 \\
\hline $\begin{array}{l}\text { RTSE- } \\
\text { M14 }\end{array}$ & $\begin{array}{l}\text { number of unauthorized changes to system } \\
\text { assets, by life-cycle phase }\end{array}$ & $\begin{array}{l}\text { asset change } \\
\text { management }\end{array}$ & impl & $\begin{array}{l}\text { base of } \\
\text { type } \\
\text { count }\end{array}$ & RTSE:SG3.SP1 \\
\hline $\begin{array}{l}\text { RTSE- } \\
\text { M15 }\end{array}$ & $\begin{array}{l}\text { inspection yield: defects found during the } \\
\text { inspection / (defects found during the in- } \\
\text { spection + those that escaped the inspec- } \\
\text { tion) }\end{array}$ & inspections & $\begin{array}{l}\text { effective- } \\
\text { ness }\end{array}$ & derived & RTSE:SG3.SP2 \\
\hline $\begin{array}{l}\text { RTSE- } \\
\text { M16 }\end{array}$ & $\begin{array}{l}\text { inspection removal rate: effort spent in in- } \\
\text { spection / number of defects found in in- } \\
\text { spection }\end{array}$ & inspections & $\begin{array}{l}\text { effective- } \\
\text { ness }\end{array}$ & derived & RTSE:SG3.SP2 \\
\hline $\begin{array}{l}\text { RTSE- } \\
\text { M17 }\end{array}$ & $\begin{array}{l}\text { planned versus actual number of inspec- } \\
\text { tions }\end{array}$ & inspections & impl & derived & RTSE:SG3.SP2 \\
\hline $\begin{array}{l}\text { RTSE- } \\
\text { M18 }\end{array}$ & $\begin{array}{l}\text { percentage of software assets released into } \\
\text { production without consideration of resi- } \\
\text { lience guidelines }\end{array}$ & $\begin{array}{l}\text { resilience guide- } \\
\text { lines for released } \\
\text { software }\end{array}$ & $\begin{array}{l}\text { impl; pos- } \\
\text { sibly effec- } \\
\text { tiveness }\end{array}$ & derived & RTSE:SG3.SP2 \\
\hline $\begin{array}{l}\text { RTSE- } \\
\text { M19 }\end{array}$ & $\begin{array}{l}\text { percentage of system assets released into } \\
\text { production without consideration of resi- } \\
\text { lience guidelines }\end{array}$ & $\begin{array}{l}\text { resilience guide- } \\
\text { lines for released } \\
\text { software }\end{array}$ & $\begin{array}{l}\text { impl; pos- } \\
\text { sibly effec- } \\
\text { tiveness }\end{array}$ & derived & RTSE:SG3.SP2 \\
\hline $\begin{array}{l}\text { RTSE- } \\
\text { M20 }\end{array}$ & $\begin{array}{l}\text { elapsed time between the identification of a } \\
\text { newly released software or system asset } \\
\text { and its inclusion in the asset inventory }\end{array}$ & asset inventory & $\begin{array}{l}\text { impl; pos- } \\
\text { sibly effec- } \\
\text { tiveness }\end{array}$ & $\begin{array}{l}\text { base of } \\
\text { type } \\
\text { sche- } \\
\text { dule }\end{array}$ & ADM:SG1.SP1 \\
\hline $\begin{array}{l}\text { RTSE- } \\
\text { M21 }\end{array}$ & $\begin{array}{l}\text { number of software and system develop- } \\
\text { ment risks referred to the risk management } \\
\text { process }\end{array}$ & risk identification & impl & $\begin{array}{l}\text { base of } \\
\text { type } \\
\text { count }\end{array}$ & RTSE:SG3.SP1 \\
\hline $\begin{array}{l}\text { RTSE- } \\
\text { M22 }\end{array}$ & $\begin{array}{l}\text { percentage of software and system devel- } \\
\text { opment policies that are met }\end{array}$ & policy & impl & derived & none \\
\hline $\begin{array}{l}\text { RTSE- } \\
\text { M23 }\end{array}$ & $\begin{array}{l}\text { test defect density (number of vulnerabilities } \\
\text { found in test / size of software asset) }\end{array}$ & system test & impl & derived & RTSE.SG1.SP4 \\
\hline $\begin{array}{l}\text { RTSE- } \\
\text { M24 }\end{array}$ & $\begin{array}{l}\text { usage defect density (number of vulnerabili- } \\
\text { ties found while using software or number of } \\
\text { incidents that occurred while using software } \\
\text { / size of software asset) }\end{array}$ & $\begin{array}{l}\text { integration test; } \\
\text { acceptance test; } \\
\text { usage }\end{array}$ & impl & derived & RTSE.SG1.SP5 \\
\hline
\end{tabular}

\section{Service Continuity (SC)}

The purpose of Service Continuity is to ensure the continuity of essential operations of services and related assets if a disruption occurs as a result of an incident, disaster, or other disruptive event.

\section{Summary of Specific Goals and Practices}

SC:SG1 Prepare for Service Continuity

SC:SG1.SP1 Plan for Service Continuity

SC:SG1.SP2 Establish Standards and Guidelines for Service Continuity

SC:SG2 Identify and Prioritize High-Value Services 
SC:SG2.SP1 Identify the Organization's High-Value Services

SC:SG2.SP2 Identify Internal and External Dependencies and Interdependencies

SC:SG2.SP3 Identify Vital Organizational Records and Databases

SC:SG3 Develop Service Continuity Plans

SC:SG3.SP1 Identify Plans to Be Developed

SC:SG3.SP2 Develop and Document Service Continuity Plans

SC:SG3.SP3 Assign Staff to Service Continuity Plans

SC:SG3.SP4 Store and Secure Service Continuity Plans

SC:SG3.SP5 Develop Service Continuity Plan Training

SC:SG4 Validate Service Continuity Plans

SC:SG4.SP1 Validate Plans to Requirements and Standards

SC:SG4.SP2 Identify and Resolve Plan Conflicts

SC:SG5 Exercise Service Continuity Plans

SC:SG5.SP1 Develop Testing Program and Standards

SC:SG5.SP2 Develop and Document Test Plans

$\mathrm{SC}: \mathrm{SG} 5 . \mathrm{SP} 3$ Exercise Plans

SC:SG5.SP4 Evaluate Plan Test Results

SC:SG6 Execute Service Continuity Plans

SC:SG6.SP1 Execute Plans

SC:SG6.SP2 Measure the Effectiveness of the Plans in Operation

SC:SG7 Maintain Service Continuity Plans

SC:SG7.SP1 Establish Change Criteria

SC:SG7.SP2 Maintain Changes to Plans

Measures

\begin{tabular}{|c|c|c|c|c|c|}
\hline ID & Measure & $\begin{array}{l}\text { Type of } \\
\text { Information }\end{array}$ & $\begin{array}{l}\text { Measure } \\
\text { Type }\end{array}$ & $\begin{array}{l}\text { Base or } \\
\text { Derived }\end{array}$ & $\begin{array}{l}\text { Applicable } \\
\text { SG.SP }\end{array}$ \\
\hline SC-M1 & $\begin{array}{l}\text { elapsed time since the organization-wide plan } \\
\text { for managing SC and the standards and } \\
\text { guidelines for SC were reviewed and updated }\end{array}$ & SC program & impl & $\begin{array}{l}\text { base of } \\
\text { type sche- } \\
\text { dule }\end{array}$ & $\begin{array}{l}\text { SC:SG1.SP1 } \\
\text { SC:SG1.SP2 }\end{array}$ \\
\hline SC-M2 & $\begin{array}{l}\text { percentage of unstaffed roles and responsi- } \\
\text { bilities in the organization-wide plan for man- } \\
\text { aging SC }\end{array}$ & SC program & impl & derived & SC:SG1.SP1 \\
\hline SC-M3 & $\begin{array}{l}\text { percentage of SC guidelines and standards } \\
\text { that are more/less stringent than required to } \\
\text { meet compliance obligations }\end{array}$ & SC program & impl & derived & $\begin{array}{l}\text { SC:SG1.SP1 } \\
\text { SC:SG1.SP2 }\end{array}$ \\
\hline SC-M4 & $\begin{array}{l}\text { number of relationships }{ }^{45} \text { (organization-wide, } \\
\text { by SC plan) necessary to ensure SC }\end{array}$ & SC program & impl & $\begin{array}{l}\text { base of } \\
\text { type count }\end{array}$ & SC:SG2.SP2 \\
\hline SC-M5 & $\begin{array}{l}\text { number of points of contact for relationships } \\
\text { that require updates }\end{array}$ & SC program & impl & $\begin{array}{l}\text { base of } \\
\text { type count }\end{array}$ & SC:SG2.SP2 \\
\hline SC-M6 & $\begin{array}{l}\text { elapsed time since review and update of the } \\
\text { list of vital organizational records and data- } \\
\text { bases }\end{array}$ & SC program & impl & $\begin{array}{l}\text { base of } \\
\text { type sche- } \\
\text { dule }\end{array}$ & SC:SG2.SP3 \\
\hline
\end{tabular}

45 Internal dependencies, external dependencies, and interdependencies (refer to SC:SG2.SP2) 


\begin{tabular}{|c|c|c|c|c|c|}
\hline ID & Measure & $\begin{array}{l}\text { Type of } \\
\text { Information }\end{array}$ & $\begin{array}{l}\text { Measure } \\
\text { Type }\end{array}$ & $\begin{array}{l}\text { Base or } \\
\text { Derived }\end{array}$ & $\begin{array}{l}\text { Applicable } \\
\text { SG.SP }\end{array}$ \\
\hline SC-M7 & percentage of SC plans completed & $\begin{array}{l}\text { SC plan devel- } \\
\text { opment }\end{array}$ & impl & derived & SC:SG3.SP2 \\
\hline SC-M8 & $\begin{array}{l}\text { number of required SC plans that have not yet } \\
\text { been developed (based on high-value servic- } \\
\text { es and associated assets that do not have SC } \\
\text { plans) }\end{array}$ & $\begin{array}{l}\text { SC plan devel- } \\
\text { opment }\end{array}$ & impl & $\begin{array}{l}\text { base of } \\
\text { type count }\end{array}$ & $\begin{array}{l}\text { SC:SG2.SP1 } \\
\text { SC:SG3.SP1 }\end{array}$ \\
\hline SC-M9 & $\begin{array}{l}\text { percentage of SC plans that are not stored in } \\
\text { a central storage system }\end{array}$ & $\begin{array}{l}\text { SC plan devel- } \\
\text { opment }\end{array}$ & impl & derived & SC:SG3.SP4 \\
\hline $\begin{array}{l}\text { SC- } \\
\text { M10 }\end{array}$ & $\begin{array}{l}\text { percentage of plans that are dependent on } \\
\text { other plans; number of plans on which they } \\
\text { are dependent }\end{array}$ & $\begin{array}{l}\text { SC plan depen- } \\
\text { dencies }\end{array}$ & impl & $\begin{array}{l}\text { derived; } \\
\text { base of } \\
\text { type count }\end{array}$ & SC:SG4.SP2 \\
\hline $\begin{array}{l}\text { SC- } \\
\text { M11 }\end{array}$ & $\begin{array}{l}\text { percentage of plans with missing components } \\
\text { (designated owner, resources, etc.) }\end{array}$ & $\begin{array}{l}\text { SC plan omis- } \\
\text { sions }\end{array}$ & impl & derived & SC:SG3.SP2 \\
\hline $\begin{array}{l}\text { SC- } \\
\text { M12 }\end{array}$ & $\begin{array}{l}\text { percentage of plans without established own- } \\
\text { ers }\end{array}$ & $\begin{array}{l}\text { SC plan omis- } \\
\text { sions }\end{array}$ & impl & derived & SC:SG3.SP2 \\
\hline $\begin{array}{l}\text { SC- } \\
\text { M13 }\end{array}$ & $\begin{array}{l}\text { percentage of plans without identified stake- } \\
\text { holders }\end{array}$ & $\begin{array}{l}\text { SC plan omis- } \\
\text { sions }\end{array}$ & impl & derived & SC:SG3.SP2 \\
\hline $\begin{array}{l}\text { SC- } \\
\text { M14 }\end{array}$ & $\begin{array}{l}\text { number of staff assigned to SC plans that are } \\
\text { no longer employed by the organization }\end{array}$ & $\begin{array}{l}\text { SC plan omis- } \\
\text { sions }\end{array}$ & impl & $\begin{array}{l}\text { base of } \\
\text { type count }\end{array}$ & SC:SG3.SP3 \\
\hline SC-M1 & $\begin{array}{l}\text { percentage of defined roles in SC plans that } \\
\text { are not assigned to specific staff }\end{array}$ & $\begin{array}{l}\text { SC plan omis- } \\
\text { sions }\end{array}$ & impl & derived & SC:SG3.SP3 \\
\hline $\begin{array}{l}\text { SC- } \\
\text { M15 }\end{array}$ & $\begin{array}{l}\text { percentage of defined roles in SC plans for } \\
\text { which backup staff are not identified }\end{array}$ & $\begin{array}{l}\text { SC plan omis- } \\
\text { sions }\end{array}$ & impl & derived & SC:SG3.SP3 \\
\hline $\begin{array}{l}\text { SC- } \\
\text { M16 }\end{array}$ & $\begin{array}{l}\text { percentage of SC plans that do not meet } \\
\text { service and asset resilience requirements }\end{array}$ & $\begin{array}{l}\text { SC plan omis- } \\
\text { sions }\end{array}$ & impl & derived & SC:SG4.SP1 \\
\hline $\begin{array}{l}\text { SC- } \\
\text { M17 }\end{array}$ & $\begin{array}{l}\text { percentage of SC plans that do not meet } \\
\text { standards and guidelines }\end{array}$ & $\begin{array}{l}\text { SC plan omis- } \\
\text { sions }\end{array}$ & impl & derived & SC:SG4.SP1 \\
\hline $\begin{array}{l}\text { SC- } \\
\text { M18 }\end{array}$ & $\begin{array}{l}\text { percentage of staff not covered by a service } \\
\text { continuity plan }\end{array}$ & $\begin{array}{l}\text { SC plan omis- } \\
\text { sions }\end{array}$ & impl & derived & none \\
\hline $\begin{array}{l}\text { SC- } \\
\text { M19 }\end{array}$ & $\begin{array}{l}\text { percentage of staff who have not been trained } \\
\text { on their roles and responsibilities as defined } \\
\text { in SC plans }\end{array}$ & SC plan training & impl & derived & SC:SG3.SP5 \\
\hline $\begin{array}{l}\text { SC- } \\
\text { M20 }\end{array}$ & $\begin{array}{l}\text { percentage of plans with one or more severe } \\
\text { conflicts (such as a single point of failure) that } \\
\text { have not been mitigated }\end{array}$ & $\begin{array}{l}\text { SC plan con- } \\
\text { flicts }\end{array}$ & impl & derived & SC:SG4.SP2 \\
\hline $\begin{array}{l}\text { SC- } \\
\text { M21 }\end{array}$ & $\begin{array}{l}\text { percentage of SC plans that do not have a } \\
\text { schedule for testing and review }\end{array}$ & SC plan testing & impl & derived & SC:SG5.SP1 \\
\hline $\begin{array}{l}\text { SC- } \\
\text { M22 }\end{array}$ & $\begin{array}{l}\text { percentage of SC plans that do not have a } \\
\text { test plan }\end{array}$ & SC plan testing & impl & derived & SC:SG5.SP2 \\
\hline $\begin{array}{l}\text { SC- } \\
\text { M23 }\end{array}$ & $\begin{array}{l}\text { percentage of SC test plans that have/have } \\
\text { not been exercised }\end{array}$ & $\mathrm{SC}$ plan testing & impl & derived & SC:SG5.SP3 \\
\hline $\begin{array}{l}\text { SC- } \\
\text { M24 }\end{array}$ & $\begin{array}{l}\text { percentage of interdependent service continu- } \\
\text { ity plans that have/have not been jointly } \\
\text { tested }\end{array}$ & SC plan testing & impl & derived & SC:SG5.SP3 \\
\hline $\begin{array}{l}\text { SC- } \\
\text { M25 }\end{array}$ & $\begin{array}{l}\text { percentage of SC test plans that have failed } \\
\text { one or more test objectives }\end{array}$ & SC plan testing & impl & derived & SC:SG5.SP4 \\
\hline $\begin{array}{l}\text { SC- } \\
\text { M26 }\end{array}$ & $\begin{array}{l}\text { percentage of SC plan test objectives (RTOs } \\
\text { and RPOs) unmet }\end{array}$ & SC plan testing & impl & derived & SC:SG5.SP4 \\
\hline $\begin{array}{l}\text { SC- } \\
\text { M27 }\end{array}$ & $\begin{array}{l}\text { number of staff with defined roles in SC plans } \\
\text { who do not have access to such plans within } \\
\text { specified thresholds (time) }\end{array}$ & SC plan testing & impl & $\begin{array}{l}\text { base of } \\
\text { type count }\end{array}$ & SC:SG3.SP4 \\
\hline $\begin{array}{l}\text { SC- } \\
\text { M28 }\end{array}$ & $\begin{array}{l}\text { average time for staff with defined SC plan } \\
\text { roles to access SC plans }\end{array}$ & SC plan testing & impl & $\begin{array}{l}\text { base of } \\
\text { type sche- } \\
\text { dule }\end{array}$ & $\begin{array}{l}\text { SC:SG3.SP4 } \\
\text { SC:SG5.SP3 }\end{array}$ \\
\hline
\end{tabular}




\begin{tabular}{|l|l|l|l|l|l|}
\hline ID & Measure & $\begin{array}{l}\text { Type of } \\
\text { Information }\end{array}$ & $\begin{array}{l}\text { Measure } \\
\text { Type }\end{array}$ & $\begin{array}{l}\text { Base or } \\
\text { Derived }\end{array}$ & $\begin{array}{l}\text { Applicable } \\
\text { SG.SP }\end{array}$ \\
\hline $\begin{array}{l}\text { SC- } \\
\text { M29 }\end{array}$ & $\begin{array}{l}\text { percentage of realized risks for service conti- } \\
\text { nuity that exceed established risk parameters }\end{array}$ & SC risk & $\begin{array}{l}\text { effec- } \\
\text { tiveness }\end{array}$ & derived & none \\
\hline $\begin{array}{l}\text { SC- } \\
\text { M30 }\end{array}$ & $\begin{array}{l}\text { percentage of SC plans executed (never ex- } \\
\text { ecuted) }\end{array}$ & $\begin{array}{l}\text { SC plan execu- } \\
\text { tion }\end{array}$ & impl & $\begin{array}{l}\text { base of } \\
\text { type count; } \\
\text { derived }\end{array}$ & SC:SG6.SP1 \\
\hline $\begin{array}{l}\text { SC- } \\
\text { M31 }\end{array}$ & $\begin{array}{l}\text { percentage of plans that have not been re- } \\
\text { viewed post-execution }\end{array}$ & SC plan review & impl & derived & SC:SG6.SP2 \\
\hline $\begin{array}{l}\text { SC- } \\
\text { M32 }\end{array}$ & $\begin{array}{l}\text { percentage of plans that require changes (as } \\
\text { defined by change criteria) }\end{array}$ & $\begin{array}{l}\text { SC plan } \\
\text { changes }\end{array}$ & impl & derived & $\begin{array}{l}\text { SC:SG7.SP1 } \\
\text { SC:SG7.SP2 }\end{array}$ \\
\hline $\begin{array}{l}\text { SC- } \\
\text { M33 }\end{array}$ & $\begin{array}{l}\text { percentage of plans that have been changed } \\
\text { without authorization }\end{array}$ & $\begin{array}{l}\text { SC plan } \\
\text { changes }\end{array}$ & impl & derived & SC:SG7.SP2 \\
\hline $\begin{array}{l}\text { SC- } \\
\text { M34 }\end{array}$ & $\begin{array}{l}\text { percentage of plans that have been changed } \\
\text { without review }\end{array}$ & $\begin{array}{l}\text { SC plan } \\
\text { changes }\end{array}$ & impl & derived & SC:SG7.SP2 \\
\hline $\begin{array}{l}\text { SC- } \\
\text { M35 }\end{array}$ & $\begin{array}{l}\text { percentage of plans that have been changed } \\
\text { without testing }\end{array}$ & $\begin{array}{l}\text { SC plan } \\
\text { changes }\end{array}$ & impl & derived & SC:SG7.SP2 \\
\hline $\begin{array}{l}\text { SC- } \\
\text { M36 }\end{array}$ & $\begin{array}{l}\text { frequency of changes to plans by service or } \\
\text { service type }\end{array}$ & $\begin{array}{l}\text { SC plan } \\
\text { changes }\end{array}$ & impl & $\begin{array}{l}\text { base of } \\
\text { type sche- } \\
\text { dule }\end{array}$ & SC:SG7.SP2 \\
\hline
\end{tabular}

\section{Technology Management (TM)}

The purpose of Technology Management is to establish and manage an appropriate level of controls related to the integrity and availability of technology assets to support the resilient operations of organizational services.

\section{Summary of Specific Goals and Practices}

TM:SG1 Establish and Prioritize Technology Assets

TM:SG1.SP1 Prioritize Technology Assets

TM:SG1.SP2 Establish Resilience-Focused Technology Assets

TM:SG2 Protect Technology Assets

TM:SG2.SP1 Assign Resilience Requirements to Technology Assets

TM:SG2.SP2 Establish and Implement Controls

TM:SG3 Manage Technology Asset Risk

TM:SG3.SP1 Identify and Assess Technology Asset Risk

TM:SG3.SP2 Mitigate Technology Risk

TM:SG4 Manage Technology Asset Integrity

TM:SG4.SP1 Control Access to Technology Assets

TM:SG4.SP2 Perform Configuration Management

TM:SG4.SP3 Perform Change Control and Management

TM:SG4.SP4 Perform Release Management

TM:SG5 Manage Technology Asset Availability

TM:SG5.SP1 Perform Planning to Sustain Technology Assets

TM:SG5.SP2 Manage Technology Asset Maintenance

TM:SG5.SP3 Manage Technology Capacity 
TM:SG5.SP4 Manage Technology Interoperability

Measures

\begin{tabular}{|c|c|c|c|c|c|}
\hline ID & Measure & $\begin{array}{l}\text { Type of } \\
\text { Information }\end{array}$ & $\begin{array}{l}\text { Measure } \\
\text { Type }\end{array}$ & $\begin{array}{l}\text { Base or } \\
\text { Derived }\end{array}$ & $\begin{array}{l}\text { Applicable } \\
\text { SG.SP }\end{array}$ \\
\hline TM-M1 & $\begin{array}{l}\text { percentage of technology assets that have } \\
\text { been inventoried }\end{array}$ & asset inventory & impl & derived & ADM SG1.SP1 \\
\hline TM-M2 & $\begin{array}{l}\text { percentage of technology assets with/without } \\
\text { a complete asset profile (such as no stated } \\
\text { resilience requirements) }\end{array}$ & asset inventory & impl & derived & $\begin{array}{l}\text { ADM:SG1.SP2 } \\
\text { TM:SG2.SP1 }\end{array}$ \\
\hline TM-M3 & $\begin{array}{l}\text { percentage of technology assets with/without } \\
\text { a designated owner }\end{array}$ & asset inventory & impl & derived & ADM:SG1.SP3 \\
\hline TM-M4 & $\begin{array}{l}\text { percentage of technology assets with/without } \\
\text { a designated custodian }\end{array}$ & asset inventory & impl & derived & ADM:SG1.SP3 \\
\hline TM-M5 & $\begin{array}{l}\text { percentage of technology assets that have } \\
\text { designated owners but no custodians }\end{array}$ & asset inventory & impl & derived & ADM:SG1.SP3 \\
\hline TM-M6 & $\begin{array}{l}\text { percentage of technology assets that have } \\
\text { designated custodians but no owners }\end{array}$ & asset inventory & impl & derived & ADM:SG1.SP3 \\
\hline TM-M7 & $\begin{array}{l}\text { percentage of technology assets that have } \\
\text { been inventoried, by service }\end{array}$ & asset inventory & impl & derived & ADM:SG2.SP1 \\
\hline TM-M8 & $\begin{array}{l}\text { percentage of technology assets that are not } \\
\text { associated with one or more services }\end{array}$ & asset inventory & impl & derived & ADM:SG2.SP1 \\
\hline TM-M9 & $\begin{array}{l}\text { percentage of technology asset-service de- } \\
\text { pendency conflicts with unimplemented or } \\
\text { incomplete mitigation plans }\end{array}$ & $\begin{array}{l}\text { asset-service } \\
\text { dependencies }\end{array}$ & impl & derived & ADM:SG2.SP2 \\
\hline TM-M10 & $\begin{array}{l}\text { percentage of technology asset-service de- } \\
\text { pendency conflicts with no mitigation plans }\end{array}$ & $\begin{array}{l}\text { asset-service } \\
\text { dependencies }\end{array}$ & impl & derived & $\begin{array}{l}\text { ADM:SG2.SP2 } \\
\text { TM:SG2.SP2 }\end{array}$ \\
\hline TM-M11 & $\begin{array}{l}\text { number of discrepancies between the current } \\
\text { inventory and the previous inventory }\end{array}$ & asset inventory & impl & $\begin{array}{l}\text { base of } \\
\text { type } \\
\text { count }\end{array}$ & ADM SG3.SP1 \\
\hline TM-M12 & $\begin{array}{l}\text { number of changes made to asset profiles in } \\
\text { the technology asset inventory }\end{array}$ & asset inventory & impl & $\begin{array}{l}\text { base of } \\
\text { type } \\
\text { count }\end{array}$ & ADM SG3.SP2 \\
\hline TM-M13 & $\begin{array}{l}\text { number of changes to resilience requirements } \\
\text { as a result of technology asset changes }\end{array}$ & $\begin{array}{l}\text { asset change } \\
\text { management }\end{array}$ & impl & $\begin{array}{l}\text { base of } \\
\text { type } \\
\text { count }\end{array}$ & ADM:SG3.SP2 \\
\hline TM-M14 & $\begin{array}{l}\text { number of changes to service continuity plans } \\
\text { as a result of technology asset changes }\end{array}$ & $\begin{array}{l}\text { asset change } \\
\text { management }\end{array}$ & impl & $\begin{array}{l}\text { base of } \\
\text { type } \\
\text { count }\end{array}$ & ADM:SG3.SP2 \\
\hline TM-M15 & $\begin{array}{l}\text { percentage of technology assets that are } \\
\text { designated as high-value assets }\end{array}$ & asset inventory & impl & derived & TM:SG1.SP1 \\
\hline TM-M16 & $\begin{array}{l}\text { elapsed time since the technology asset in- } \\
\text { ventory was last reviewed }\end{array}$ & asset inventory & impl & $\begin{array}{l}\text { base of } \\
\text { type } \\
\text { schedule }\end{array}$ & $\begin{array}{l}\text { ADM:SG1.SP1 } \\
\text { ADM:SG3.SP1 }\end{array}$ \\
\hline TM-M17 & $\begin{array}{l}\text { elapsed time since review and validation of } \\
\text { high-value technology assets and their priori- } \\
\text { ties }\end{array}$ & asset inventory & impl & $\begin{array}{l}\text { base of } \\
\text { type } \\
\text { schedule }\end{array}$ & TM:SG1.SP1 \\
\hline TM-M18 & $\begin{array}{l}\text { elapsed time since review and reconciliation } \\
\text { of resilience-focused technology assets (those } \\
\text { required for service continuity \& service resto- } \\
\text { ration) }\end{array}$ & asset inventory & impl & $\begin{array}{l}\text { base of } \\
\text { type } \\
\text { schedule }\end{array}$ & TM:SG1.SP2 \\
\hline
\end{tabular}




\begin{tabular}{|c|c|c|c|c|c|}
\hline ID & Measure & $\begin{array}{l}\text { Type of } \\
\text { Information }\end{array}$ & $\begin{array}{l}\text { Measure } \\
\text { Type }\end{array}$ & $\begin{array}{l}\text { Base or } \\
\text { Derived }\end{array}$ & $\begin{array}{l}\text { Applicable } \\
\text { SG.SP }\end{array}$ \\
\hline TM-M19 & $\begin{array}{l}\text { percentage of technology assets without as- } \\
\text { signed/defined resilience requirements }\end{array}$ & $\begin{array}{l}\text { asset require- } \\
\text { ments }\end{array}$ & impl & derived & TM:SG2.SP1 \\
\hline TM-M20 & $\begin{array}{l}\text { percentage of technology assets with as- } \\
\text { signed/defined resilience requirements that } \\
\text { are undocumented }\end{array}$ & $\begin{array}{l}\text { asset require- } \\
\text { ments }\end{array}$ & impl & derived & TM:SG2.SP1 \\
\hline TM-M21 & $\begin{array}{l}\text { percentage of technology assets that do not } \\
\text { satisfy their resilience requirements }\end{array}$ & $\begin{array}{l}\text { asset require- } \\
\text { ment }\end{array}$ & impl & derived & TM:SG2.SP1 \\
\hline TM-M22 & $\begin{array}{l}\text { percentage of technology assets with no or } \\
\text { missing protection controls }\end{array}$ & asset controls & $\begin{array}{l}\text { impl; } \\
\text { possibly } \\
\text { effec- } \\
\text { tiveness }\end{array}$ & derived & TM:SG2.SP2 \\
\hline TM-M23 & $\begin{array}{l}\text { percentage of technology assets with no or } \\
\text { missing sustainment controls }\end{array}$ & asset controls & $\begin{array}{l}\text { impl; } \\
\text { possibly } \\
\text { effec- } \\
\text { tiveness }\end{array}$ & derived & TM:SG2.SP2 \\
\hline TM-M24 & $\begin{array}{l}\text { percentage of technology asset controls (pro- } \\
\text { tection and sustainment) that are ineffective or } \\
\text { inadequate as demonstrated by: } \\
\text { - unsatisfied control objectives } \\
\text { - unmet resilience requirements } \\
\text { - outstanding control assessment problem } \\
\text { areas above established thresholds and } \\
\text { without remediation plans }\end{array}$ & asset controls & $\begin{array}{l}\text { impl; } \\
\text { possibly } \\
\text { effec- } \\
\text { tiveness }\end{array}$ & derived & TM:SG2.SP2 \\
\hline TM-M25 & $\begin{array}{l}\text { percentage of technology asset control defi- } \\
\text { ciencies not resolved by scheduled due date } \\
\text { (refer to CTRL measures for categories of } \\
\text { control deficiencies) }\end{array}$ & asset controls & $\mathrm{impl}$ & derived & TM:SG2.SP2 \\
\hline TM-M26 & $\begin{array}{l}\text { elapsed time since review of the effectiveness } \\
\text { of technology asset controls }\end{array}$ & asset controls & impl & $\begin{array}{l}\text { base of } \\
\text { type } \\
\text { schedule }\end{array}$ & TM:SG2.SP2 \\
\hline TM-M27 & $\begin{array}{l}\text { elapsed time since risk assessment of tech- } \\
\text { nology assets performed }\end{array}$ & asset risk & impl & $\begin{array}{l}\text { base of } \\
\text { type } \\
\text { schedule }\end{array}$ & TM:SG3.SP1 \\
\hline TM-M28 & $\begin{array}{l}\text { elapsed time since business impact analysis } \\
\text { of technology assets performed }\end{array}$ & asset risk & impl & $\begin{array}{l}\text { base of } \\
\text { type } \\
\text { schedule }\end{array}$ & TM:SG3.SP1 \\
\hline TM-M29 & $\begin{array}{l}\text { percentage of technology assets for which } \\
\text { business impact valuation }{ }^{46} \text { has not been } \\
\text { performed }\end{array}$ & asset risk & impl & derived & TM:SG3.SP1 \\
\hline TM-M30 & $\begin{array}{l}\text { percentage of technology assets for which a } \\
\text { risk assessment has not been performed and } \\
\text { documented (per policy or other guideline) } \\
\text { and according to plan }\end{array}$ & asset risk & impl & derived & $\begin{array}{l}\text { TM:SG3.SP1 } \\
\text { TM:SG5.SP4 } \\
\text { subpractice } 3\end{array}$ \\
\hline TM-M31 & $\begin{array}{l}\text { percentage of technology asset risks that } \\
\text { have not been assigned to a responsible party } \\
\text { for action, tracking, and closure }\end{array}$ & asset risk & impl & derived & TM:SG3.SP2 \\
\hline TM-M32 & $\begin{array}{l}\text { percentage of technology asset risks }{ }^{47} \text { with a } \\
\text { disposition of "mitigate or control" that do not } \\
\text { have a defined mitigation plan }\end{array}$ & asset risk & impl & derived & TM:SG3.SP2 ${ }^{48}$ \\
\hline
\end{tabular}

46 Business impact valuation can be either qualitative (high, medium, low) or quantitative (based on levels of loss or damage, fines, number of customers lost, disruption in access, etc.)

47 This measure also appears in RISK M4-1. For ease of use of an individual PA (vs. ease of maintenance and consistency), we have decided to replicate some (but not all) risk-related measures in the individual asset PAs that are identified generally in the list of RISK PA measures.

48 SG3.SP2 subpractice 7 states, "Collect performance measures on the risk management process." No such measures are included here in TM; refer to the RISK PA. 


\begin{tabular}{|c|c|c|c|c|c|}
\hline ID & Measure & $\begin{array}{l}\text { Type of } \\
\text { Information }\end{array}$ & $\begin{array}{l}\text { Measure } \\
\text { Type }\end{array}$ & $\begin{array}{l}\text { Base or } \\
\text { Derived }\end{array}$ & $\begin{array}{l}\text { Applicable } \\
\text { SG.SP }\end{array}$ \\
\hline TM-M33 & $\begin{array}{l}\text { percentage of technology asset risks with a } \\
\text { "mitigate or control" disposition that are not } \\
\text { effectively mitigated by their mitigation plans }\end{array}$ & asset risk & $\begin{array}{l}\text { effec- } \\
\text { tiveness }\end{array}$ & derived & TM:SG3.SP2 \\
\hline TM-M34 & $\begin{array}{l}\text { percentage of realized risks for technology } \\
\text { assets that exceed established risk parame- } \\
\text { ters }\end{array}$ & asset risk & $\begin{array}{l}\text { effec- } \\
\text { tiveness }\end{array}$ & derived & TM:SG3.SP2 \\
\hline TM-M35 & $\begin{array}{l}\text { number of violations of access control policies } \\
\text { for technology assets }\end{array}$ & $\begin{array}{l}\text { asset access } \\
\text { controls }\end{array}$ & impl & $\begin{array}{l}\text { base of } \\
\text { type } \\
\text { count }\end{array}$ & TM:SG4.SP1 \\
\hline TM-M36 & $\begin{array}{l}\text { percentage of intrusions into digital technology } \\
\text { assets where impact exceeds threshold }\end{array}$ & asset intrusions & impl & derived & TM:SG4.SP1 \\
\hline TM-M37 & $\begin{array}{l}\text { percentage of intrusions into physical technol- } \\
\text { ogy assets where impact exceeds threshold }\end{array}$ & asset intrusions & impl & derived & TM:SG4.SP1 \\
\hline TM-M38 & $\begin{array}{l}\text { elapsed time since audit of technology asset } \\
\text { modification logs }\end{array}$ & $\begin{array}{l}\text { asset access } \\
\text { controls }\end{array}$ & impl & $\begin{array}{l}\text { base of } \\
\text { type } \\
\text { schedule }\end{array}$ & TM:SG4.SP1 \\
\hline TM-M39 & $\begin{array}{l}\text { percentage of technology assets for which } \\
\text { approved configuration settings have/have not } \\
\text { been implemented as required by policy }\end{array}$ & $\begin{array}{l}\text { asset configu- } \\
\text { ration }\end{array}$ & impl & derived & TM:SG4.SP2 \\
\hline TM-M40 & $\begin{array}{l}\text { percentage of technology assets with configu- } \\
\text { rations that deviate from approved standards } \\
\text { for which exceptions have not been granted }\end{array}$ & $\begin{array}{l}\text { asset configu- } \\
\text { ration }\end{array}$ & impl & derived & TM:SG4.SP2 \\
\hline TM-M41 & $\begin{array}{l}\text { elapsed time since review of technology asset } \\
\text { configuration control logs }\end{array}$ & $\begin{array}{l}\text { asset configu- } \\
\text { ration }\end{array}$ & impl & $\begin{array}{l}\text { base of } \\
\text { type } \\
\text { schedule }\end{array}$ & TM:SG4.SP2 \\
\hline TM-M42 & $\begin{array}{l}\text { elapsed time since audit of technology asset } \\
\text { configurations }\end{array}$ & $\begin{array}{l}\text { asset configu- } \\
\text { ration }\end{array}$ & impl & $\begin{array}{l}\text { base of } \\
\text { type } \\
\text { schedule }\end{array}$ & TM:SG4.SP2 \\
\hline TM-M43 & $\begin{array}{l}\text { number of unauthorized changes to technolo- } \\
\text { gy assets (may need to report by some mea- } \\
\text { ningful categorization of assets) }\end{array}$ & $\begin{array}{l}\text { asset change } \\
\text { management }\end{array}$ & impl & $\begin{array}{l}\text { base of } \\
\text { type } \\
\text { count }\end{array}$ & TM:SG4.SP3 \\
\hline TM-M44 & $\begin{array}{l}\text { change success rate (percentage of changes } \\
\text { to technology assets that succeed without } \\
\text { causing an incident, service outage, or im- } \\
\text { pairment) }\end{array}$ & $\begin{array}{l}\text { asset change } \\
\text { management }\end{array}$ & impl & derived & TM:SG4.SP3 \\
\hline TM-M45 & $\begin{array}{l}\text { percentage of changes that are high-priority, } \\
\text { emergency changes }\end{array}$ & $\begin{array}{l}\text { asset change } \\
\text { management }\end{array}$ & impl & derived & TM:SG4.SP3 \\
\hline TM-M46 & $\begin{array}{l}\text { percentage of changes that result from defi- } \\
\text { ciencies in resilience requirements }\end{array}$ & $\begin{array}{l}\text { asst change } \\
\text { management }\end{array}$ & impl & derived & TM:SG4.SP3 \\
\hline TM-M47 & $\begin{array}{l}\text { elapsed time between: } \\
\text { - scheduled technology asset configuration } \\
\text { updates and actual configuration updates } \\
\text { - scheduled technology asset changes and } \\
\text { actual changes } \\
\text { - scheduled technology asset releases into } \\
\text { production and actual releases }\end{array}$ & $\begin{array}{l}\text { asset configu- } \\
\text { ration, change, } \\
\text { and release } \\
\text { management }\end{array}$ & impl & $\begin{array}{l}\text { base of } \\
\text { type } \\
\text { schedule }\end{array}$ & $\begin{array}{l}\text { TM:SG4.SP2 } \\
\text { TM:SG4.SP3 } \\
\text { TM:SG4.SP4 }\end{array}$ \\
\hline TM-M48 & $\begin{array}{l}\text { percentage of technology assets approved for } \\
\text { release into production that have not under- } \\
\text { gone a security review }\end{array}$ & $\begin{array}{l}\text { asset release } \\
\text { management }\end{array}$ & impl & derived & TM:SG4.SP4 \\
\hline TM-M49 & $\begin{array}{l}\text { percentage of technology assets released into } \\
\text { production that have not undergone security } \\
\text { testing in accordance with policy }\end{array}$ & $\begin{array}{l}\text { asset release } \\
\text { management }\end{array}$ & impl & derived & TM:SG4.SP4 \\
\hline TM-M50 & $\begin{array}{l}\text { percentage of technology assets released to } \\
\text { production that deviate from approved stan- } \\
\text { dards for which exceptions have not been } \\
\text { granted }\end{array}$ & $\begin{array}{l}\text { asset release } \\
\text { management }\end{array}$ & impl & derived & TM:SG4.SP4 \\
\hline
\end{tabular}




\begin{tabular}{|c|c|c|c|c|c|}
\hline ID & Measure & $\begin{array}{l}\text { Type of } \\
\text { Information }\end{array}$ & $\begin{array}{l}\text { Measure } \\
\text { Type }\end{array}$ & $\begin{array}{l}\text { Base or } \\
\text { Derived }\end{array}$ & $\begin{array}{l}\text { Applicable } \\
\text { SG.SP }\end{array}$ \\
\hline TM-M51 & $\begin{array}{l}\text { percentage of technology assets without } \\
\text { availability metrics }\end{array}$ & $\begin{array}{l}\text { asset sustain- } \\
\text { ment }\end{array}$ & impl & derived & TM:SG5.SP1 \\
\hline TM-M52 & $\begin{array}{l}\text { percentage of technology assets without re- } \\
\text { covery time objectives (RTO) }\end{array}$ & $\begin{array}{l}\text { asset sustain- } \\
\text { ment }\end{array}$ & impl & derived & TM:SG5.SP1 \\
\hline TM-M53 & $\begin{array}{l}\text { percentage of technology assets without re- } \\
\text { covery point objectives (RPO) }\end{array}$ & $\begin{array}{l}\text { asset sustain- } \\
\text { ment }\end{array}$ & impl & derived & TM:SG5.SP1 \\
\hline TM-M54 & $\begin{array}{l}\text { number of technology assets that do not have } \\
\text { their own service continuity plan where one is } \\
\text { required }\end{array}$ & $\begin{array}{l}\text { asset sustain- } \\
\text { ment }\end{array}$ & $\mathrm{impl}$ & $\begin{array}{l}\text { base of } \\
\text { type } \\
\text { count }\end{array}$ & TM:SG5.SP1 \\
\hline TM-M55 & $\begin{array}{l}\text { percentage of external entities that are not } \\
\text { meeting service level agreements for technol- } \\
\text { ogy assets subject to external entity services }\end{array}$ & $\begin{array}{l}\text { asset sustain- } \\
\text { ment }\end{array}$ & impl & derived & none \\
\hline TM-M56 & $\begin{array}{l}\text { elapsed time since technology asset mainten- } \\
\text { ance performed }\end{array}$ & $\begin{array}{l}\text { asset mainten- } \\
\text { ance }\end{array}$ & impl & $\begin{array}{l}\text { base of } \\
\text { type } \\
\text { schedule }\end{array}$ & TM:SG5.SP2 \\
\hline TM-M57 & $\begin{array}{l}\text { number of scheduled maintenance activities } \\
\text { that exceed recommended service intervals }\end{array}$ & $\begin{array}{l}\text { asset mainten- } \\
\text { ance }\end{array}$ & impl & $\begin{array}{l}\text { base of } \\
\text { type } \\
\text { count }\end{array}$ & TM:SG5.SP2 \\
\hline TM-M58 & $\begin{array}{l}\text { number of scheduled maintenance activities } \\
\text { that do not meet recommended specifications }\end{array}$ & $\begin{array}{l}\text { asset mainten- } \\
\text { ance }\end{array}$ & impl & $\begin{array}{l}\text { base of } \\
\text { type } \\
\text { count }\end{array}$ & TM:SG5.SP2 \\
\hline TM-M59 & $\begin{array}{l}\text { number of maintenance changes that were } \\
\text { made without following change management } \\
\text { procedures }\end{array}$ & $\begin{array}{l}\text { asset mainten- } \\
\text { ance }\end{array}$ & impl & $\begin{array}{l}\text { base of } \\
\text { type } \\
\text { count }\end{array}$ & TM:SG5.SP2 \\
\hline TM-M60 & $\begin{array}{l}\text { number of technology assets requiring capaci- } \\
\text { ty management for which no forecast or strat- } \\
\text { egy exists }\end{array}$ & asset capacity & impl & $\begin{array}{l}\text { base of } \\
\text { type } \\
\text { count }\end{array}$ & TM:SG5.SP3 \\
\hline TM-M61 & $\begin{array}{l}\text { elapsed time since the capacity management } \\
\text { strategy for technology assets has been vali- } \\
\text { dated and updated }\end{array}$ & asset capacity & $\mathrm{impl}$ & $\begin{array}{l}\text { base of } \\
\text { type } \\
\text { schedule }\end{array}$ & TM:SG5.SP3 \\
\hline TM-M62 & $\begin{array}{l}\text { elapsed time since the technology asset inte- } \\
\text { roperability strategy has been reviewed }\end{array}$ & asset capacity & impl & $\begin{array}{l}\text { base of } \\
\text { type } \\
\text { schedule }\end{array}$ & TM:SG5.SP4 \\
\hline
\end{tabular}

\section{Vulnerability Analysis and Resolution (VAR)}

The purpose of Vulnerability Analysis and Resolution is to identify, analyze, and manage vulnerabilities in an organization's operating environment.

\section{Summary of Specific Goals and Practices}

VAR:SG1 Prepare for Vulnerability Analysis and Resolution

\section{VAR:SG1.SP1 Establish Scope}

VAR:SG1.SP2 Establish a Vulnerability Analysis and Resolution Strategy

VAR:SG2 Identify and Analyze Vulnerabilities

VAR:SG2.SP1 Identify Sources of Vulnerability Information

VAR:SG2.SP2 Discover Vulnerabilities

VAR:SG2.SP3 Analyze Vulnerabilities

VAR:SG3 Manage Exposure to Vulnerabilities

VAR:SG3.SP1 Manage Exposure to Vulnerabilities 
VAR:SG4 Identify Root Causes

VAR:SG4.SP1 Perform Root-Cause Analysis

\section{Measures}

\begin{tabular}{|c|c|c|c|c|c|}
\hline ID & Measure & $\begin{array}{l}\text { Type of } \\
\text { Information }\end{array}$ & $\begin{array}{l}\text { Measure } \\
\text { Type }\end{array}$ & $\begin{array}{l}\text { Base or } \\
\text { Derived }\end{array}$ & $\begin{array}{l}\text { Applicable } \\
\text { SG.SP }\end{array}$ \\
\hline $\begin{array}{l}\text { VAR- } \\
\text { M1 }\end{array}$ & $\begin{array}{l}\text { percentage of high-value assets (by type or } \\
\text { category) subject to VAR process activities } \\
\text { (This is determined by the resilience require- } \\
\text { ments associated with assets and assumes } \\
\text { an up-to-date asset inventory [refer to ADM].) }\end{array}$ & $\begin{array}{l}\text { asset inventory; } \\
\text { ORMS scope }\end{array}$ & impl & derived & $\begin{array}{l}\text { ADM:SG1.SP1 } \\
\text { ADM:SG1.SP2 }\end{array}$ \\
\hline $\begin{array}{l}\text { VAR- } \\
\text { M2 }\end{array}$ & $\begin{array}{l}\text { percentage of high-value assets that have } \\
\text { been monitored for vulnerabilities within an } \\
\text { agreed-upon time interval }\end{array}$ & vul monitoring & impl & derived & $\begin{array}{l}\text { ADM:SG1.SP1 } \\
\text { MON:SG2.SP3 }\end{array}$ \\
\hline $\begin{array}{l}\text { VAR- } \\
\text { M3 }\end{array}$ & $\begin{array}{l}\text { percentage of high-value assets that have } \\
\text { been audited or assessed for vulnerabilities }\end{array}$ & vul assessment & impl & derived & $\begin{array}{l}\text { ADM:SG1.SP1 } \\
\text { VAR:SG2.SP2 }\end{array}$ \\
\hline $\begin{array}{l}\text { VAR- } \\
\text { M4 }\end{array}$ & $\begin{array}{l}\text { percentage of reported vulnerabilities (by } \\
\text { asset type or category) that require some } \\
\text { form of resolution or remediation (course of } \\
\text { action, reduction, elimination) }\end{array}$ & vul resolution & impl & derived & VAR:SG2.SP3 \\
\hline $\begin{array}{l}\text { VAR- } \\
\text { M5 }\end{array}$ & $\begin{array}{l}\text { percentage of vulnerabilities that have been } \\
\text { satisfactorily remediated }\end{array}$ & vul resolution & $\begin{array}{l}\text { effec- } \\
\text { tiveness }\end{array}$ & derived & VAR:SG3.SP1 \\
\hline $\begin{array}{l}\text { VAR- } \\
\text { M6 }\end{array}$ & percentage of open vulnerabilities & vul resolution & impl & derived & VAR:SG3.SP1 \\
\hline $\begin{array}{l}\text { VAR- } \\
\text { M7 }\end{array}$ & $\begin{array}{l}\text { percentage of vulnerabilities that require reso- } \\
\text { lution for which a vulnerability management } \\
\text { strategy exists }\end{array}$ & vul resolution & impl & derived & VAR:SG3.SP1 \\
\hline $\begin{array}{l}\text { VAR- } \\
\text { M8 }\end{array}$ & $\begin{array}{l}\text { percentage of vulnerabilities with vulnerability } \\
\text { management strategies that are on track per } \\
\text { plan }\end{array}$ & vul resolution & impl & derived & VAR:SG3.SP1 \\
\hline $\begin{array}{l}\text { VAR- } \\
\text { M9 }\end{array}$ & $\begin{array}{l}\text { percentage of vulnerabilities requiring a root- } \\
\text { cause analysis }\end{array}$ & vul analysis & impl & derived & VAR:SG4.SP1 \\
\hline $\begin{array}{l}\text { VAR- } \\
\text { M10 }\end{array}$ & $\begin{array}{l}\text { number of vulnerabilities that result in inci- } \\
\text { dents for which a root-cause analysis was not } \\
\text { performed }\end{array}$ & vul analysis & impl & $\begin{array}{l}\text { base of } \\
\text { type } \\
\text { count }\end{array}$ & VAR:SG4.SP1 \\
\hline $\begin{array}{l}\text { VAR- } \\
\text { M11 }\end{array}$ & $\begin{array}{l}\text { number of vulnerabilities referred to the inci- } \\
\text { dent management and control process }\end{array}$ & $\begin{array}{l}\text { incident analy- } \\
\text { sis }\end{array}$ & impl & $\begin{array}{l}\text { base of } \\
\text { type } \\
\text { count }\end{array}$ & none \\
\hline $\begin{array}{l}\text { VAR- } \\
\text { M12 }\end{array}$ & $\begin{array}{l}\text { number of vulnerabilities referred to the ser- } \\
\text { vice continuity process }\end{array}$ & $\begin{array}{l}\mathrm{SC} \text { require- } \\
\text { ments }\end{array}$ & impl & $\begin{array}{l}\text { base of } \\
\text { type } \\
\text { count }\end{array}$ & none \\
\hline $\begin{array}{l}\text { VAR- } \\
\text { M13 }\end{array}$ & $\begin{array}{l}\text { elapsed time from high-value vulnerability } \\
\text { data collection to data distribution to key } \\
\text { stakeholders }\end{array}$ & $\begin{array}{l}\text { vul communica- } \\
\text { tion }\end{array}$ & $\begin{array}{l}\text { effec- } \\
\text { tiveness }\end{array}$ & $\begin{array}{l}\text { base of } \\
\text { type } \\
\text { schedule }\end{array}$ & VAR:GG2.GP7 \\
\hline $\begin{array}{l}\text { VAR- } \\
\text { M14 }\end{array}$ & $\begin{array}{l}\text { number of vulnerabilities referred to the risk } \\
\text { management process }\end{array}$ & $\begin{array}{l}\text { risk identifica- } \\
\text { tion }\end{array}$ & impl & $\begin{array}{l}\text { base of } \\
\text { type } \\
\text { count }\end{array}$ & VAR:SG2.SP3 \\
\hline $\begin{array}{l}\text { VAR- } \\
\text { M15 }\end{array}$ & $\begin{array}{l}\text { percentage of organizational units, lines of } \\
\text { business, and services using vulnerability } \\
\text { data to assess the performance of operational } \\
\text { resilience management processes }\end{array}$ & $\begin{array}{l}\text { ORMS assess- } \\
\text { ment }\end{array}$ & $\begin{array}{l}\text { process } \\
\text { perfor- } \\
\text { mance }\end{array}$ & derived & none \\
\hline
\end{tabular}




\section{References}

URLs are valid as of the publication date of this document.

\section{[Allen 2010]}

Allen, Julia \& Davis, Noopur. Measuring Operational Resilience Using the CERT Resilience Management Model (CMU/SEI-2010-TN-030). Software Engineering Institute, Carnegie Mellon University, September 2010.

http://www.sei.cmu.edu/library/abstracts/reports/10tn030.cfm

\section{[Caralli 2010]}

Caralli, Richard A.; Allen, Julia H.; Curtis, Pamela D.; White, David W.; \& Young, Lisa R. CERT ${ }^{\circledR}$ Resilience Management Model, v1.0 (CMU/SEI-2010-TR-012). Software Engineering Institute, Carnegie Mellon University, 2010.

http://www.sei.cmu.edu/library/abstracts/reports/10tr012.cfm

\section{[Caralli 2011]}

Caralli, Richard A.; Allen, Julia H.; \& White, David W. CERT ${ }^{\circledR}$ Resilience Management Model: A Maturity Model for Managing Operational Resilience. Addison-Wesley, 2011. 




